Portland State University

PDXScholar

\title{
Development and application of some quantitative stratigraphic techniques to the Coos Bay coalfield, a Tertiary fluvio-deltaic complex in southwestern Oregon
}

Willard Sidney Titus III

Portland State University

Follow this and additional works at: https://pdxscholar.library.pdx.edu/open_access_etds

Part of the Geology Commons, Geophysics and Seismology Commons, and the Stratigraphy Commons

Let us know how access to this document benefits you.

\section{Recommended Citation}

Titus, Willard Sidney III, "Development and application of some quantitative stratigraphic techniques to the Coos Bay coalfield, a Tertiary fluvio-deltaic complex in southwestern Oregon" (1987). Dissertations and Theses. Paper 3730.

https://doi.org/10.15760/etd.5614

This Thesis is brought to you for free and open access. It has been accepted for inclusion in Dissertations and Theses by an authorized administrator of PDXScholar. Please contact us if we can make this document more accessible: pdxscholar@pdx.edu. 
AN ABSTRACT OF THE THESIS OF willard sidney Titus, III for the Master of Science in Geology presented June 2, 1987.

Title: Development and Application of some Quantitative Stratigraphic Techniques to the Coos Bay Coalfield, A Tertiary Fluvio-Deltaic Complex in southwestern oregon.

APPROVED BY MEMBERS OF THE THESIS COMMITTEE:
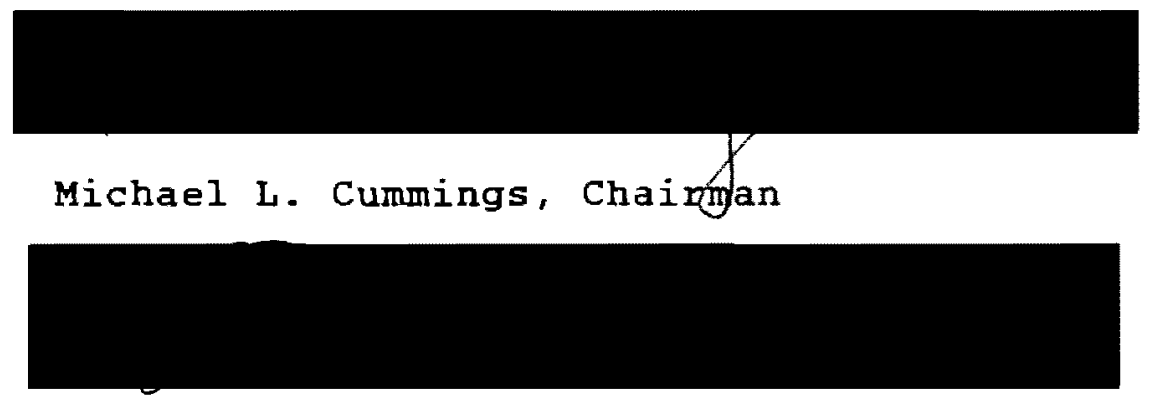

Robert 0 . Van Atta

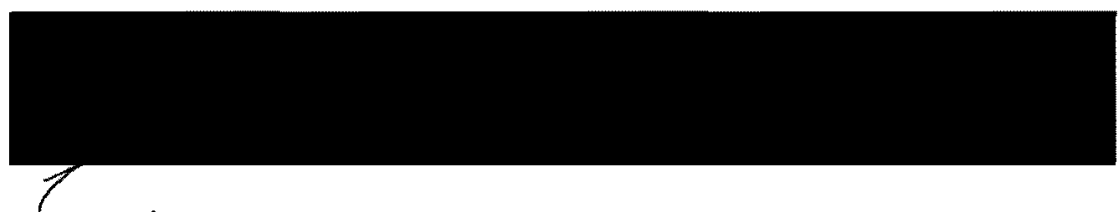

Marvin H. Beeson

A computer technique for interpreting geophysical logs of drill-holes in quantitative lithologic terms has been developed and tested on the deposits of the late Eocene Coaledo Formation, a well-studied fluvio-deltaic complex in southwestern oregon. The technique involves the use of induced and natural gamma logs for separation of coal and 
claystone from coarse-grained detrital rocks and the use of the ratio of resistivity and natural gamma responses (defined here as the "grain size index") to divide the coarse clastic rocks into a series of textural classes corresponding to the Wentworth-Udden particle size scaie.

The technique was used to examine 3,300 meters of geophysical logs at 0.33 meter intervals in 17 drill-holes penetrating the coal-bearing section of the upper member of the Coaledo Formation in the central portion of the Coos Bay coal field near Coquille, Oregon. The vertical sequences of lithologies produced by this analysis were integrated into a series of lithofacies develcjed by a first-order, embedded Markov analysis of the lithological transitions. A series of quantitative techniques were developed to use the vertical relationships between these lithofacies to model, in three-dimensions, the characteristics of the depositional system responsible for the accumulation of the complex.

The result of these procedures were subjected to statistical tests for internal consistency, for correlation against independent lithological determinations and for comparison with analogous modern depositional systems. The techniques were found to be rapid, practical and effective, producing useful, reproducible stratigraphic information with a resolution an order of magnitude better than previously available. The results of the log interpretation technique, which consist of sequence of 
quantitative textural parameters, can be treated by any of a number of mathematical and statistical techniques for smoothing, transition frequency analysis, spectral analysis and correlation. 
DEVELOPMENT AND APPLICATION OF SOME QUANTITATIVE

STRATIGRAPHIC TECHNIQUES TO THE COOS BAY COALFIELD,

A TERTIARY FLUVIO-DELTAIC COMPLEX IN SOUTHWESTERN OREGON

by

WILLARD SIDNEY TITUS, III

A thesis submitted in partial fulfillment of the requirements for the degree of

MASTER OF SCIENCE

in

GEOLOGY

Portland State University 
TO THE OFFICE OF GRADUATE STUDIES AND RESEARCH:

The members of the committee approve the thesis of Willard Sidney Titus, III presented June 2, 1987.

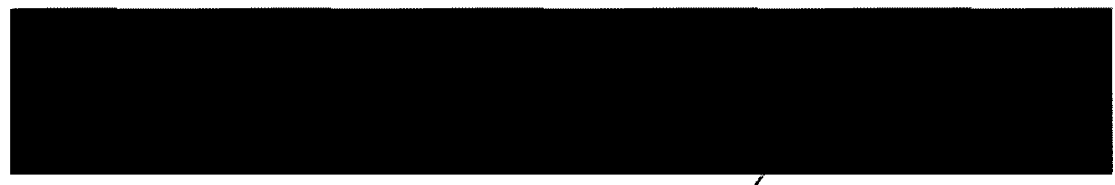

Michael L. Cummings, Chairman

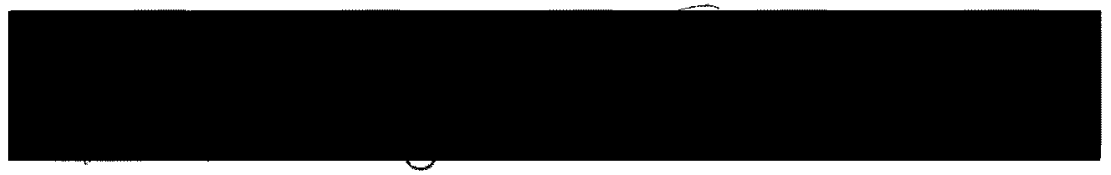

Robert O. Van Atta

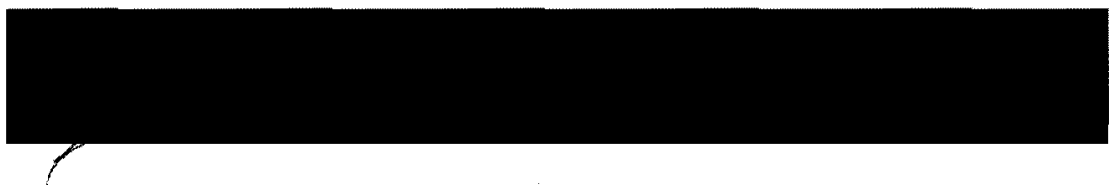

Marvin H. Beeson

APRROVED :

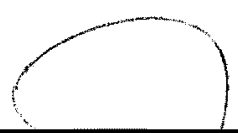

Ansel G. Johhson, Head, Geology Department

Bernard Ross, Vice Provost for Graduate studies 
TABLE OF CONTENTS

PAGE

LIST OF TABLES $\ldots \ldots \ldots \ldots \ldots \ldots \ldots \ldots \ldots \ldots \ldots \ldots \ldots \ldots$

LIST OF FIGURES $\ldots \ldots \ldots \ldots \ldots \ldots \ldots \ldots \ldots \ldots \ldots \ldots$ vi

CHAPTER

I INTRODUCTION $\ldots \ldots \ldots \ldots \ldots \ldots \ldots \ldots \ldots \ldots \ldots \ldots$

scope and Purpose $\ldots \ldots \ldots \ldots \ldots \ldots \ldots \ldots \ldots 2$

Procedures .................... 3

Geologic setting of the study area ...... 7

II PRELIMINARY INVESTIGATIONS ............ 12

Sedimentology and stratigraphy ........ 14

structural Geology ................ 24

Conclusions ................... 33

III QUANTITATIVE STRATIGRAPHY $\ldots \ldots \ldots \ldots \ldots \ldots$

Geophysical Log Interpretation ........ 37

Data Reduction .................. 53

Spatial Distribution of Lithologies ..... 57

Lithofacies Definition and Interpretation. 64

Markov Analysis of Lithofacies Transitions 70

Three-Dimensional Lithofacies Model ..... 74

Spatial Distribution of Lithofacies ..... 82

Comparison of Lithofacies Distributions .. 91 
IV CONCLUSIONS 105 


\section{LIST OF TABLES}

TABLE

PAGE

I Occurrence of major coal seams in drill

holes in the Coaledo Basin .............

II summary of lithofacies characteristics, upper

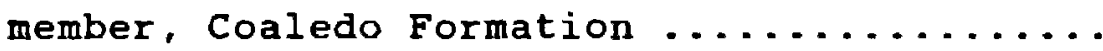

III Transition probability difference matrix,

China Creek area $\ldots \ldots \ldots \ldots \ldots \ldots \ldots \ldots$

IV Estimates of bar/channel azimuths,

China Creek area ................... 


\section{LIST OF FIGURES}

FIGURE

PAGE

1. Index to geologic studies in the coos Bay

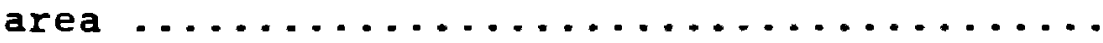

2. Location of subsurface information in the

study area $\ldots \ldots \ldots \ldots \ldots \ldots \ldots \ldots \ldots \ldots \ldots \ldots \ldots \ldots \ldots$

3. Generalized stratigraphy and lithofacies

relationships, southwestern Oregon .......

4. Regional geologic map, southwestern Oregon .....

5. Representative grain size distributions,

upper member, Coaledo Formation

6. Summary of granulometric parameters,

upper member, Coaledo Formation .........

7. QFL modal framework composition,

Coaledo and Bastendorf Formations .......

8. Stratigraphic variations in composition,

Coaledo and Bastendorf Formations ...........

9. Variability and similarity among correlative

coal seams, Coos Bay coal field .............

10. Generalized geologic map and sequential

cross-sections, Coos Bay coal field .......

11. Differential fold analysis for three major 
structural domains, Coaledo coal field .... 28

12. Structural model of faulting within the

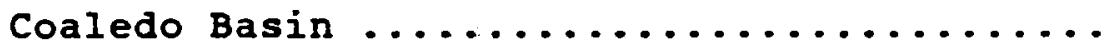

13. Generalized geologic map, China Creek area .... 36

14. Calibration of a geophysical log ........... 39

15. Classification of detrital rocks by

the grain size index technique . . . . . .

16. Relationship between modal composition

and texture . . . . . . . . . . . .

17. Comparison between grain size index and

median grain size $(M F-09)$. . . . . .

18. Calibration of grain size index to the

standard phi scale $\ldots \ldots \ldots \ldots \ldots \ldots \ldots \ldots \ldots$

19. Comparison between calculated and observed

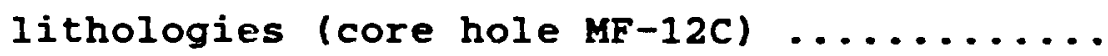
54

20. Example portion of a measured section produced

by microcomputer program "LITHLOG"

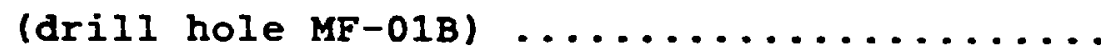

21. Lithologic character of the coal-bearing section, China Creek area $\ldots \ldots \ldots \ldots \ldots \ldots$

22. Sequential maps of coarse clastic

depocenters, China Creek area $\ldots . . \ldots \ldots \ldots$

23. Sequential maps of coal depocenters, 
China Creek area $\ldots \ldots \ldots \ldots \ldots \ldots \ldots \ldots \ldots$

24. Superimposed sand and coal depocenters,

China Creek area $\ldots \ldots \ldots \ldots \ldots \ldots \ldots \ldots \ldots$

25. Changes in coarse clastic depocenter locations

with time, China Creek area $\ldots \ldots \ldots \ldots \ldots \ldots$

65

26. Distribution of lithologies in each

lithofacies category ...............

27. Mean and standard deviation of environmental

energy index in individual lithofacies ....

28. Graphical summary of favored upwards transition

probabilities among lithofacies, China

Creek area $\ldots \ldots \ldots \ldots \ldots \ldots \ldots \ldots \ldots \ldots \ldots$

29. Mean thickness, frequency and proportions

of lithofacies, China Creek area ........ 76

30. Modeled cross-sectional relationships among

lithofacies, China creek area ..........

31. Modeled areal relationships among

lithofacies, China Creek area ..........

32. Comparison between modeled and actual

lithofacies thicknesses $\ldots \ldots \ldots \ldots \ldots \ldots$

33. Sequential maps of the actual distribution

of Iithofacies, China creek area .........

34. Linear trends of bar/channel lithofacies, 
China Creek area $\ldots \ldots \ldots \ldots \ldots \ldots \ldots \ldots \ldots \ldots$

35. Comparison of bar/channel azimuths and paleo-

current directions, China Creek area ......

36. Stratigraphic channel azimuth variations, China

Creek study area, Coos Bay coal field ......

37. Fourier series power spectrum of bar/channel

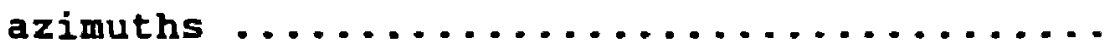

38. Three-harmonic Fourier fit to China Creek

channel azimuth data $\ldots \ldots \ldots \ldots \ldots \ldots \ldots$

39. Derived lithofacies maps constructed by

"drilling" the lithofacies model in

several orientations $\ldots \ldots \ldots \ldots \ldots \ldots \ldots \ldots$

40. Downstream channel azimuth variations,

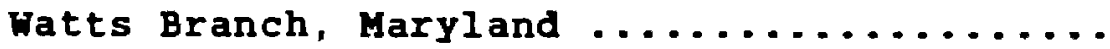

41. Thalweg azimuths, Watts Branch, Maryland .....

42. Thalweg azimuths, Lower Mississippi River near

Jackson Point, Louisiana ..............

43. Downstream variations in channel azimuths,

Lower Mississippi River ............ 100

44. Sequential development of a channel pattern,

Vermilion River, Indiana ............ 102

45. Sequential development of a flood plain,

Little Missouri River . . . . . . . . 104 
46. Example of the resolution of the quantitative stratigraphic analysis . . . . . . . 106 
CHAPTER I

INTRODUCTION

The application of quantitative methods to geological problems is developing very rapidly (Mann, 1981). Although sedimentologists were early leaders in the use of numerical and statistical methods, the difficulty of obtaining and handling large data sets has, until recently, permitted the quantitative analysis of only the simplest of stratigraphic problems. The recent availability, capacity and speed of microcomputers has led to the development of quantitative techniques suitable for application to intricate and complex stratigraphic problems (Davis, 1986). Concurrent improvements in the sophistication of geophysical logging devices (Doveton, 1986) have made an enormous amount and variety of reliable stratigraphic data available. In spite of these technical advances, little has been published regarding techniques for inferring the fundamental lithological characteristics of detrital sequences from their geophysical responses (Vaninetti, 1981) or for using vertical lithostratigraphic sequences to prepare quantitative three-dimensional depositional models. The 
value of such techniques is considerable, particularly in the fields of applied stratigraphy, petroleum and mineral exploration, hydrology and waste management.

\section{SCOPE AND PURPOSE}

The purpose of this study is to develop and test a series of quantitative techniques for:

1) interpretation of geophysical logs in fundamental lithologic terms,

2) integration of the resulting lithologic sequences into functionally related lithofacies units,

3) use of stratigraphic relationships among the resulting lithofacies to infer areal relationships, 4) use of the resulting three-dimensional lithofacies architecture to model the behavior of complex depositional systems.

Testing of the results of these techniques against one another and against independently determined parameters is an important part of the study. For that reason, the techniques were applied to a well-studied stratigraphic section, the coal-bearing strata of the upper member of the Coaledo Formation, a portion of an upper Eocene fluvio-deltaic complex exposed in the southern Coast Range of southwestern Oregon (Diller, 1899, 1901; Diller and 
Pishel, 1909; Allen and Baldwin, 1944; Duncan, 1953; Dott, 1966). The coal seams in this section are excellent stratigraphic markers in that they are readily identifiable, of considerable lateral extent, and have been correlated over considerable areas.

\section{PROCEDURES}

The study area, approximately 23 square kilometers 19 square miles) in size, is located near the town of Coquille, Oregon, 19 kilometers (12 miles) southeast of Coos Bay (Figure 1) and includes the central portion of the Coos Bay coalfield. Since the time of the last in-depth studies of the area, a number of exploratory wells have been drilled to better define the location and geometry of the coal resources. Geophysical and lithologic logs, cuttings and cores of these drill holes have been made available for this study by The Rennecott Exploration Company and The International Paper Company, joint partners in a recent re-evaluation of the coalfield. This information, coupled with the numerous measured sections, mine and prospect descriptions and structural measurements published by Allen and Baldwin (1944) and Duncan (1953) and the results of approximately four months of field work by the author forms the body of data used to test the techniques developed in this study. 


\section{INDEX TO STUDIES IN THE COOS BAY AREA}

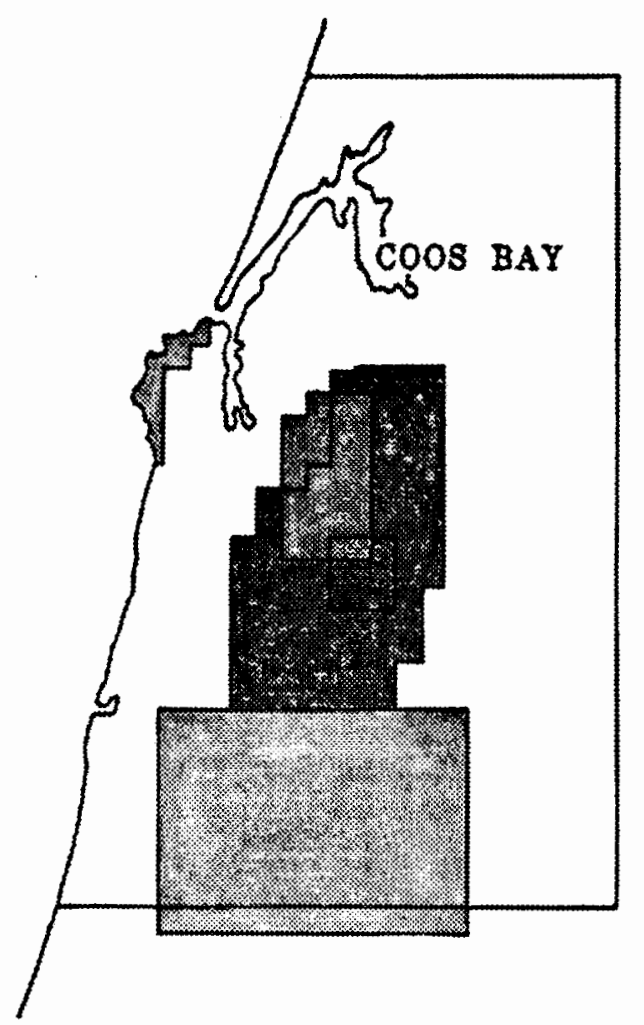

\begin{tabular}{ll}
\hline & Allen \& Baldwin, 1944 \\
Duncan, 1953 \\
Buldwin, 1969 \\
Dott, 1966 \\
Roberg, 1978 \\
Chooth, 1979 \\
Chan, 1982 \\
THLs $8 T U D Y$
\end{tabular}

Figure 1. Index to geologic studies in the coos Bay area. 
This study proceeded in three phases. In the first phase, structural and stratigraphic observations made at and near the surface throughout the Coaledo Basin were collected and correlated with first-order, qualitative stratigraphic picks of coal seams from the geophysical logs. Geologic, structure contour, and isopach maps and cross-sections based on this work were prepared and first-order structural and stratigraphic models of the study area were developed. This phase was essentially a refinement of earlier work in the area based on information obtained by additional field work and a preliminary inspection of the logs of recent drill holes. The results of this work provided a general structural and stratigraphic context for the more detailed work which follows.

The second phase involved a quantitative, foot-by-foot analysis and interpretation of the geophysical logs of a set of drill holes in the China Creek area (Figure 2). For this work, interpretive techniques were developed and tested against data obtained by granulometric analysis of samples recovered from cuttings and examination of cores removed from reference drill holes. Using these techniques, the geophysical data was converted first into lithologic sequences and subsequently into lithofacies sequences. The distribution of the lithofacies in three-dimensional space was then determined by an application of Walther's Law and conventional subsurface mapping techniques. This phase of 


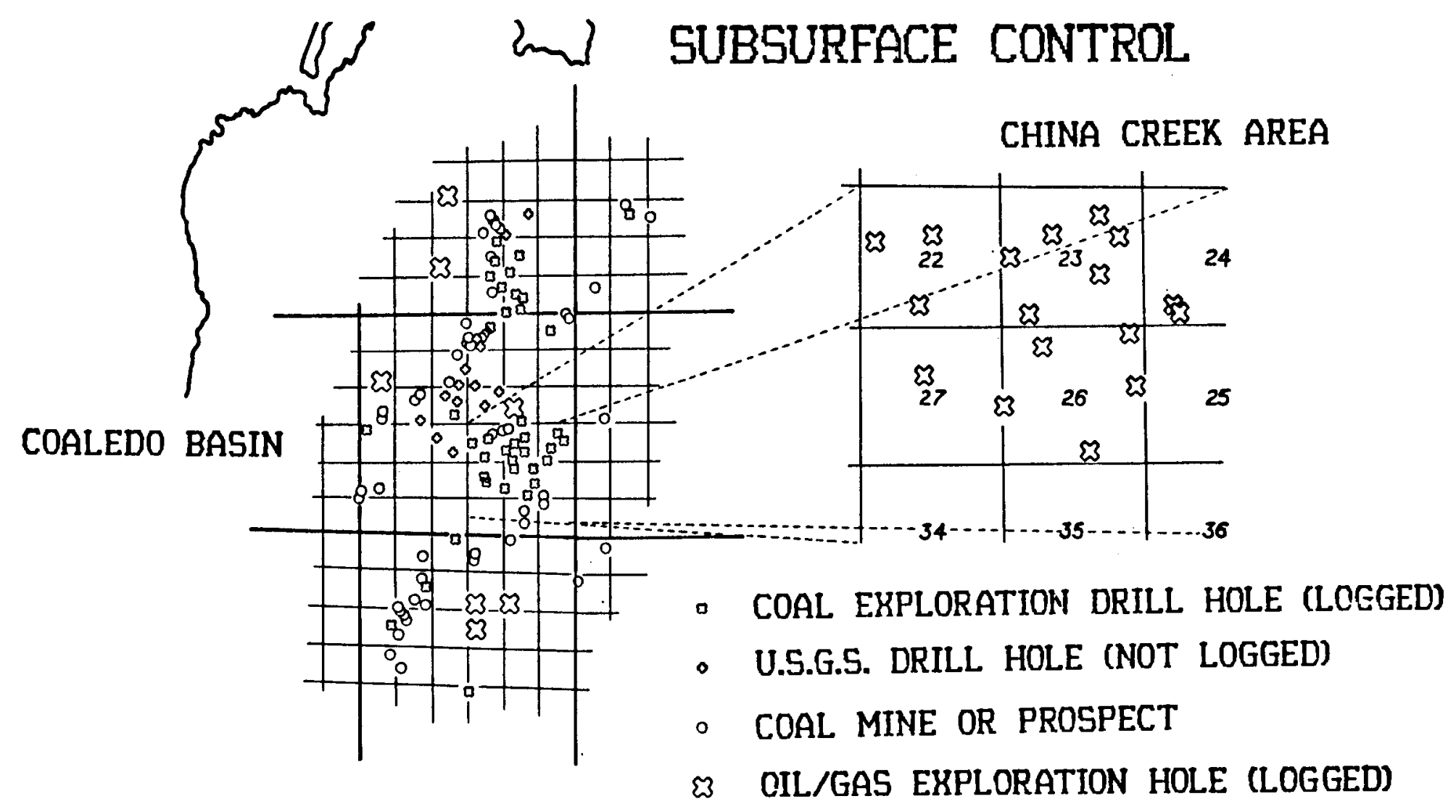

Fiqure 2. Location of subsurface information in the study area. 
the study resulted in a series of small-scale lithofacies maps which, when considered sequentially, constitute a depositional model.

The third phase of the study involved a spatial analysis of the model and a comparison of the essential characteristics of the model with independently determined parameters measured in the study area and in analogous modern depositional systems. This phase constitutes a test of the suitability and interpretative power of the techniques developed during the study.

\section{GEOLOGIC SETTING OF THE STUDY AREA}

The southern Coast Range of southwestern Oregon is composed of oceanic basalts of Paleocene and early Eocene age overlain by a thick sequence of younger Paleogene sedimentary rocks of marine, deltaic and fluvial origin (Snavely and Wagner, 1963; McKee, 1972; Baldwin, 1976; Armentrout and Suek, 1985). Although poor exposures, rapid facies changes and complicated field relationships have fostered some uncertainty and confusion in the literature describing the stratigraphy of the region, an essentially complete stratigraphic section has been assembled (Baldwin, 1976; Chan and Dott, 1986; Chan, 1982; Armentrout and others, 1983). In general, the sedimentary rocks within the province exhibit a consistent regressive trend from offshore 
turbidites deposited at bathyl depths near the base of the section, upwards through shelf and slope fan deposits to prodelta, delta front and fluvio-deltaic sediments near the top of the section (Figure 3 ).

These rocks have been deformed on several scales, most notably into three large, open, north to northeast-trending structural basins arranged in an en echelon pattern (Figure 4). These basins exhibit systematic variations in a number of fundamental characteristics, including their orientation, degree and style of deformation, and the age, thickness and general lithologic character of the rocks influenced by the structures. The easternmost basin, the Tyee Basin, is the largest and most gently folded, has the most northeasterly trending axis, and contains the oldest, most thickly bedded and most generally marine rocks. The westernmost basin, the South Slough Basin, is the smallest and most tightly folded, has the most northerly trending axis and contains the youngest, most thinly bedded and most generally terrigenous rocks. The Coaledo Basin is intermediate or transitional in each of these respects.

Within the Coaledo Basin, the section consists of the coarse-grained deltaic and fluvial deposits of the lower and upper members of the Coaledo Formation alternating with the fine grained prodelta sediments of the middle member of the Coaledo and the Bastendorf Formations (Allen and Baldwin, 

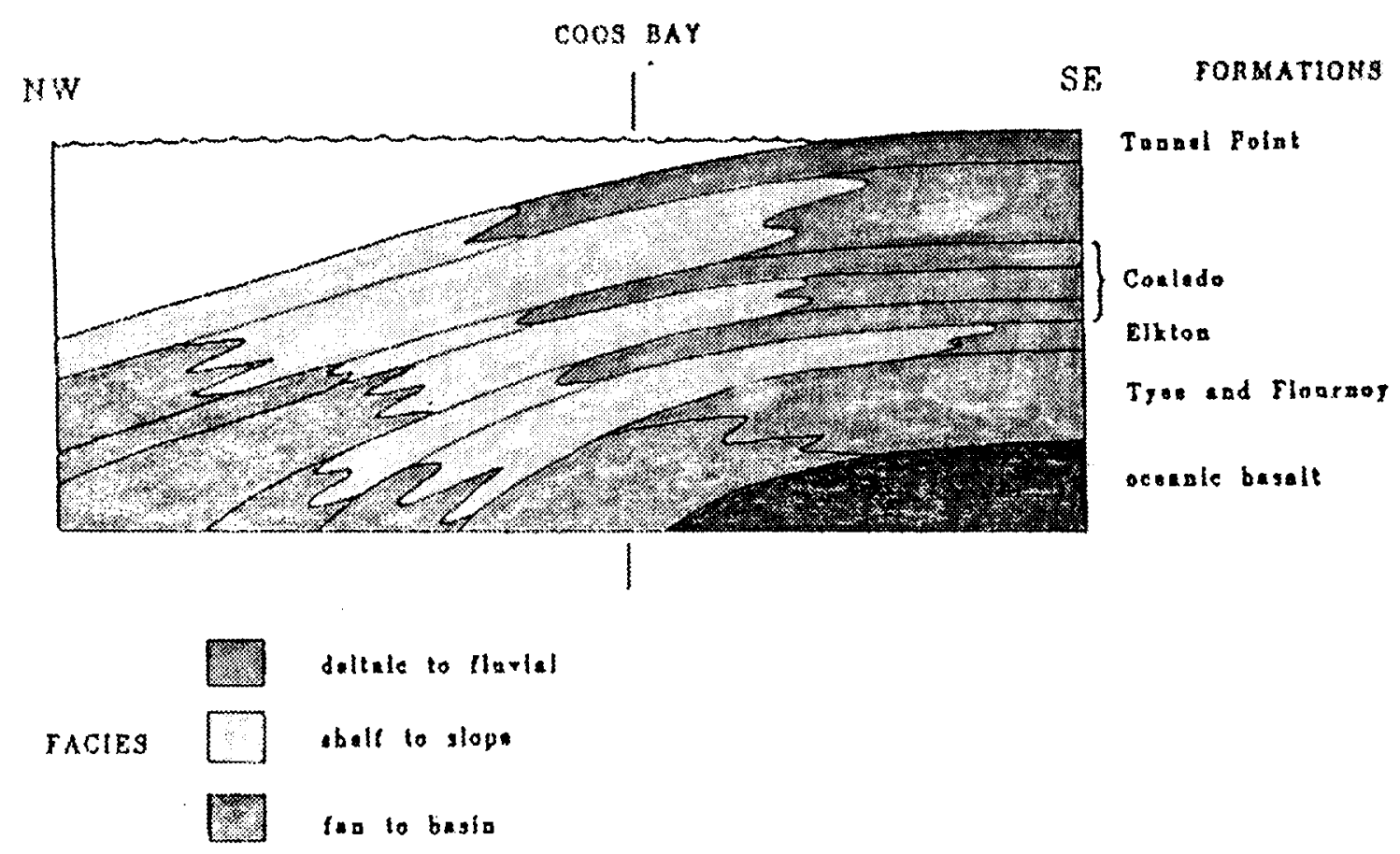

Figure 3. Generalized stratigraphy and lithofacies relationships, southwestern oregon. 


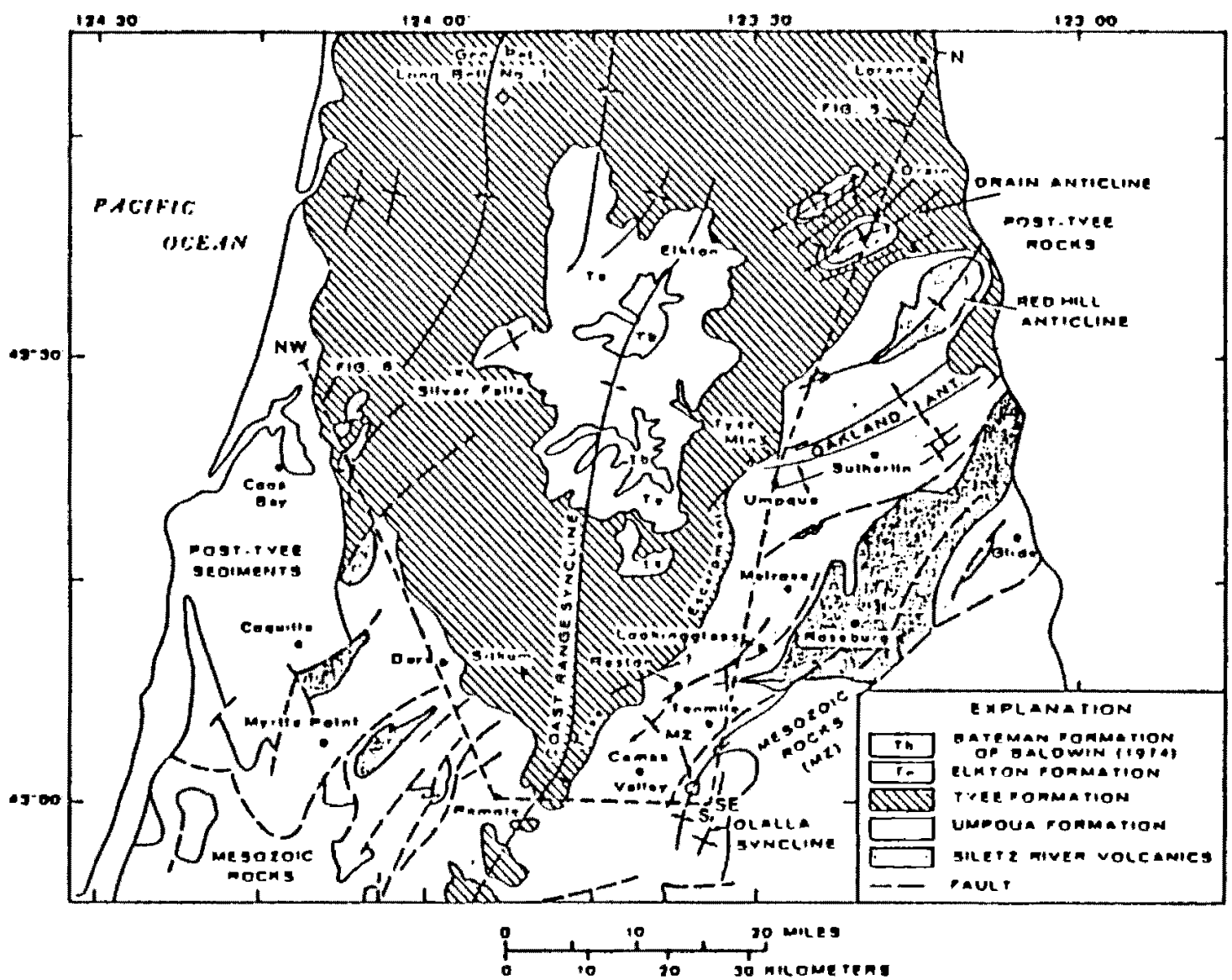

Figure 4. Regional geologic map, southwestern Oregon. 
1944; Dott, 1966; Baldwin and Beaulieu, 1973; Rooth, 1974; Ryberg, 1978; Chan, 1982). The stratigraphic position of the underlying rocks, which are exposed only on the extreme flanks of the basin and which have rarely been penetrated by drill holes, has been a subject of controversy for many years. Most recent workers have assigned them to the Elkton Formation (Dott, 1966; Rooth, 1974; Ryberg, 1978; Chan, 1982).

A major shift in provenance in the regional stratigraphic record occurs across the Elkton/Coaledo boundary. The Coaledo and Bastendorf Formations contain increasingly abundant volcaniclastic debris not present in the older strata. This material is believed to have been contributed by eruptive events in the western Cascade Range which was developing nearby during the Late Eocene and Oligocene Epochs (Heller and Ryberg, 1983).

The structural style of the Coaledo Basin is characterized by broad, deep, open folds. Structural relief of nearly one kilometer $(3,000$ feet $)$ occurs across the central portion of the basin and dips along its flanks range from 20 to 30 degrees. Faults within the basin occur on two distinct scales, one comparable to the folds in geographic extent and structural relief, the other considerably smaller. Large scale reverse faults, which are common south and east of the basin, are not known to occur within it (Allen and Baldwin, 1944, Baldwin and Beaulieu, 1973). 
CHAPTER II

\section{PRELIMINARY INVESTIGATIONS}

A preliminary study of the Coaledo Basin was conducted in order to determine:

1) whether the stratigraphic section contains appreciable erosional surfaces,

2) whether or not appreciable syndepositional deformation occurred within the basin, 3) whether the coal seams in the basin are distinct, recognizable and of sufficient continuity and lateral extent to constitute useful stratigraphic markers, 4) whether the textural and/or compositional characteristics of the clastic rocks in the area are sufficiently diverse to support a lithostratigraphic classification system

This project consisted of field mapping, analytical work and a preliminary examination of drill-hole records.

The field study involved a re-examination of exposures identified by previous workers and the location and examination of new outcrops. The general texture and composition of the rocks, the attitudes of the primary 
bedding and the nature and orientation of primary sedimentary structures were determined. Although an extensive system of newly constructed logging roads and clear-cuts facilitated access to areas not previously examined in detail, deep weathering, the tendency of the underlying bedrock to erode into a smoothly rounded topographic surface, and the rapid growth of a dense ground cover limited the exposures available for examination. Nevertheless, a network of observations covering the area in reasonable detail was established. Where extensive portions of the study area were inaccessible, low altitude stereophotographs (U.S. Department of Agriculture, 1967) of the area were examined in order to determine the general nature and attitude of the bedrock.

Since the completion of the work of Diller (1899), Diller and Pishel (1909) and Allen and Baldwin (1944), a number of exploration holes have been drilled within the Coaledo Basin. Those drilled by the U.S. Geologic Survey were continuously cored and the records of those holes consist of lithologic logs prepared at the drill site by visual inspection of the cores (Duncan, 1953). The remainder were drilled as part of an exploration program conducted jointly by the International Paper Company and The Rennecott Minerals Company. The records of these holes, which consist of mud logs, geophysical logs and core descriptions, were examined to determine the depth at which 
the major lithologic breaks and coal seams identified at the surface occurred.

\section{SEDIMENTOLOGY AND STRATIGRAPHY}

The sequence of rocks within the Coaledo Basin are divided into four lithostratigraphic units based on their overall textural character and stratigraphic position. These units, from the base of the section, are:

1) a sequence of interbedded conglomerates, sandstones, siltstones and mudstones with intercalated carbonaceous claystones and coal seams, 2) a sequence of interbedded tuffaceous siltstones and mudstones which lacks significant sandstone beds, 3) a lithostratigraphic unit which is very similar to the basal sandy unit, being separable from it only by stratigraphic position, and

4) a sequence of interbedded tuffaceous siltstones and mudstones which is similar to the lower shale, the major difference between the two being their stratigraphic position.

These units correspond to the lower, middle and upper members of the Coaledo Formation and the Bastendorf Formation described in detail by Allen and Baldwin (1944), Duncan (1953) and Chan (1982). There is no evidence pointing to appreciable unconformities at the contacts 
between these units, the boundaries being gradational over stratigraphic distances of 30 to 50 meters (100 to 150 feet).

Textures of individual strata within these units range from coarse pebble conglomerate to claystone, the variability being most pronounced in the lower and upper members of the Coaledo Formation. The results of granulometric analysis of a suite of grab samples from surface outcrops chosen to represent the textural variety of the section are summarized in Figures 5 and 6 . Although considerable variability is present, four distinct, normally distributed sub-populations can be distinguished, with break points in the neighborhood of $0.5,1.0-1.5$ and 3.5 phi. Median framework grain size ranges from 1.0 to $3.5 \mathrm{phi}$. In general, the rocks are poorly to moderately well-sorted, sorting coefficients range from 0.8 to 1.5 . Median grain size and sorting are inversely related, with a correlation coefficient of -0.78 .

Compositionally, much less variety is present. The sandstones are typically litharenites or feldspathic litharenites, with subordinate but conspicuous mica (predominantly muscovite) and carbonaceous plant debris. The most commonly occurring rock fragments are extrusive volcanics and include basalt and andesite fragments and glass shards. The composition of the siltstones and mudstones is more difficult to characterize due to the small 

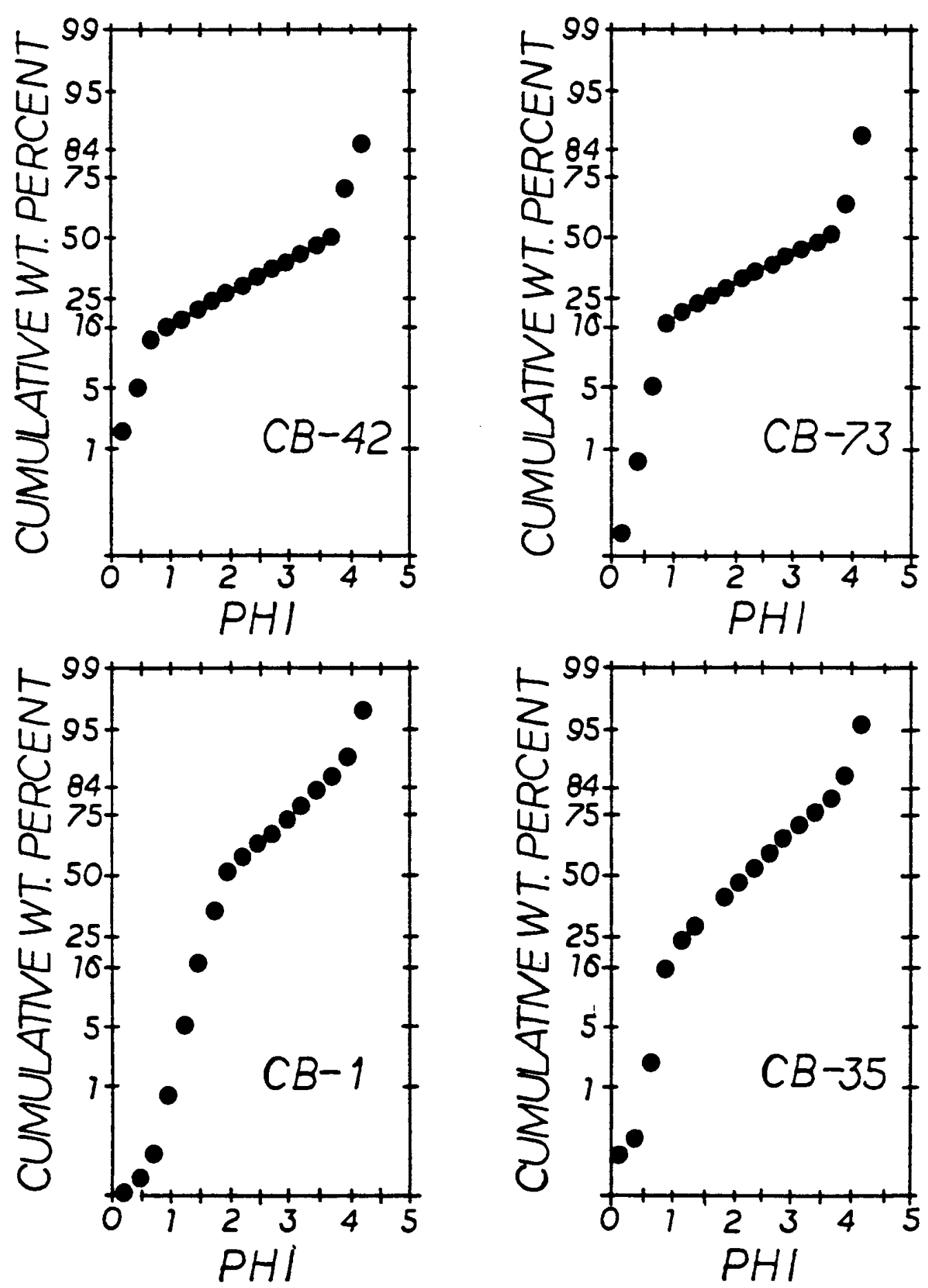

Fiqure 5. Representative grain size distributions, upper member, Coaledo Formation. 

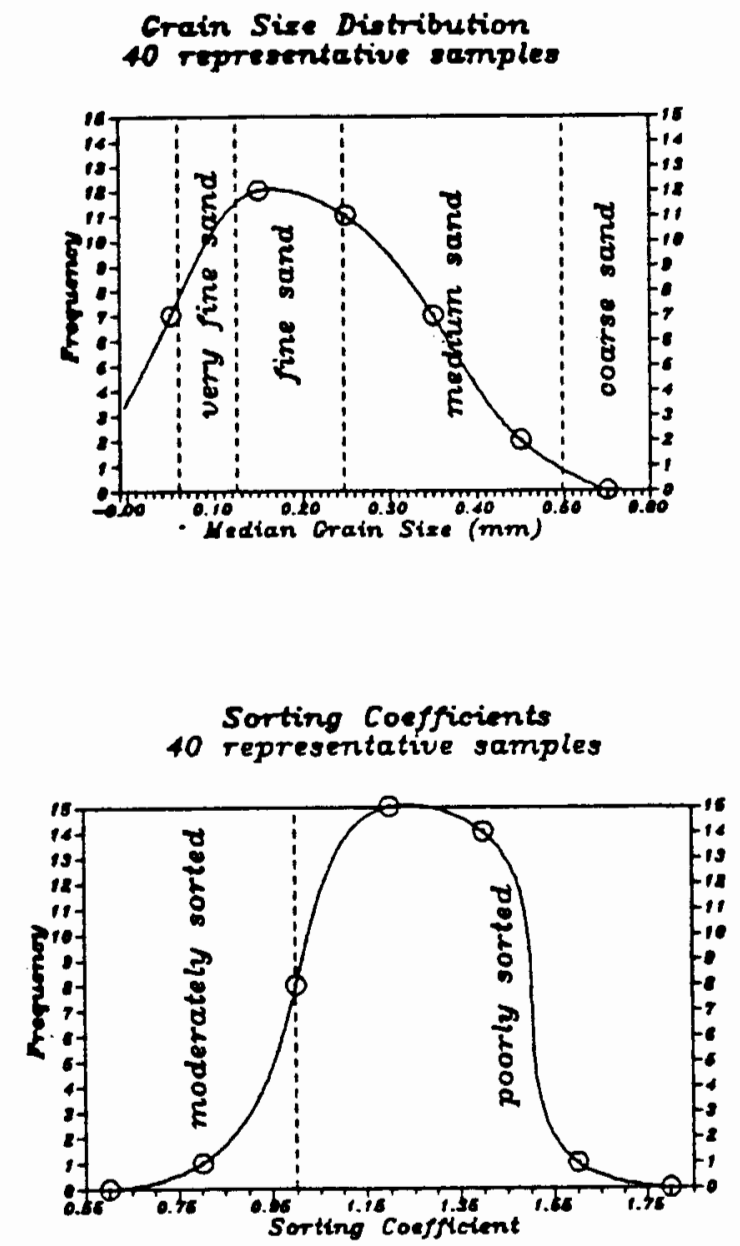

Median Grain Siee ve Sorting Coefficient 40 representative samples

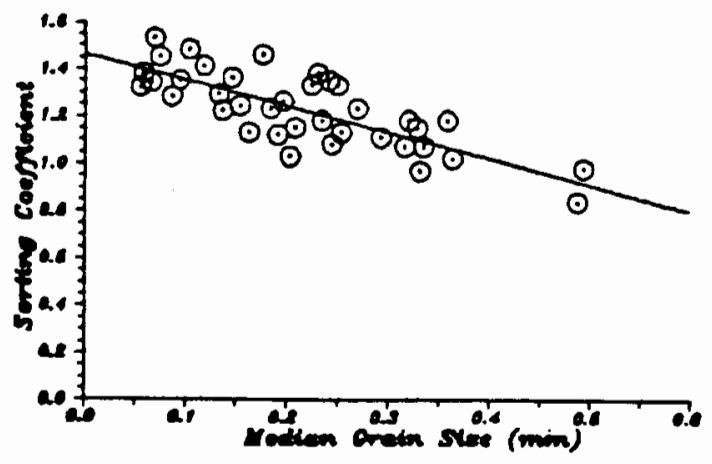

Fiqure 6. Summary of granulometric parameters, upper member, Coaledo Formation. 
size of the framework grains, but the majority are tuffaceous and rich in organic material. Specimens of similar texture collected from different stratigraphic levels and localities within the Coaledo Formation are compositionally indistinguishable. The Bastendorf Formation can be distinguished from the Coaledo Formation, as it contains a significantly higher proportion of lithic fragments. The results of a modal analysis of the medium sand fraction of 20 samples from each of the lithostratigraphic units is summarized in Figures 7 and 8 . The medium sand fraction composition averages $Q_{3} F_{3} 0 L_{3} 9$. Petrographic variation between samples collected from adjacent stratigraphic horizons at the same outcrop is often greater than that which occurs between widely separated sample sites.

Three major and a number of minor coal seams varying in thickness from a few centimeters (inches) to 10 meters (30 feet) occur within the upper member of the Coaledo Formation. The major seams consist, for the most part, of clean coal "benches" a few centimeters (inches) to a few meters (feet) thick separated from one another by thin clastic deposits (Figure 9). These "splits" are generally more tuffaceous and carbonaceous than the clastic material which constitutes the interburden strata. The proportions of the alternating coal benches and splits are consistent from one location to another within the same seam and the 


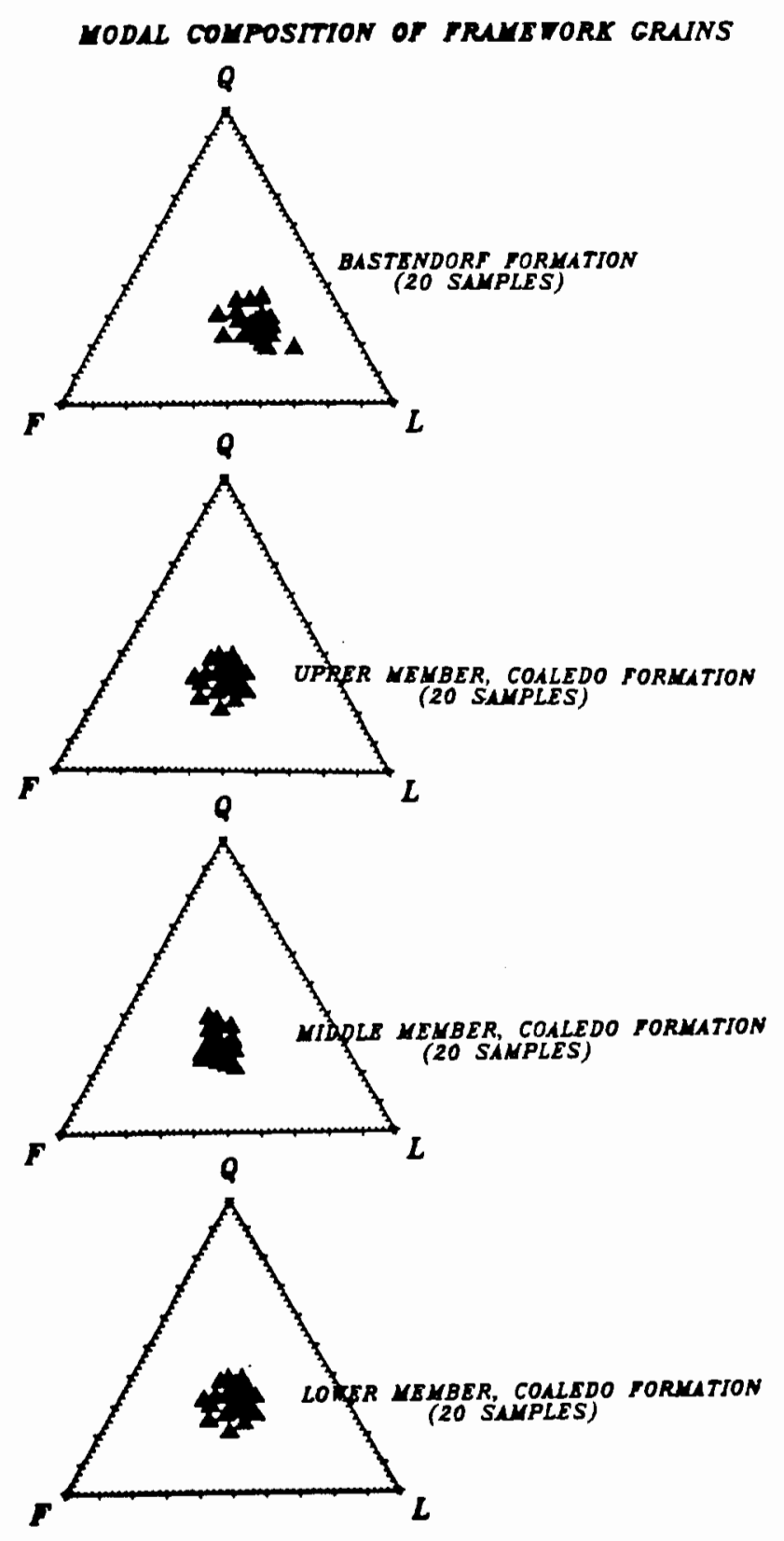

Figure 7. QFL modal framework composition, Coaledo and Bastendorf Formations. 


\section{STRATIGRAPHIC VARIATIONS IN COMPOSITION}

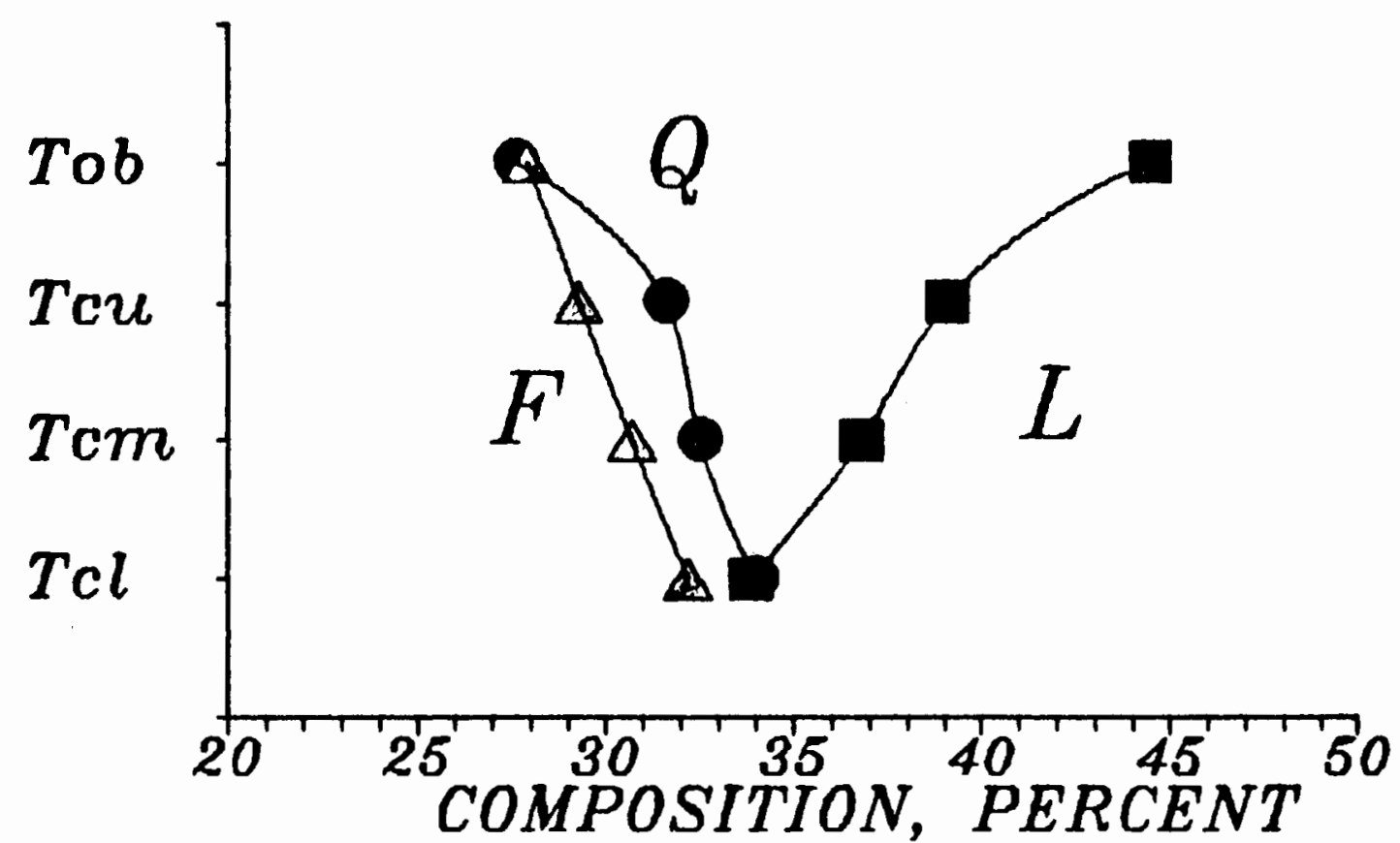

Figure 8. Stratioraphic variations in composituion, Coaledo and Bastendorf Formations. 


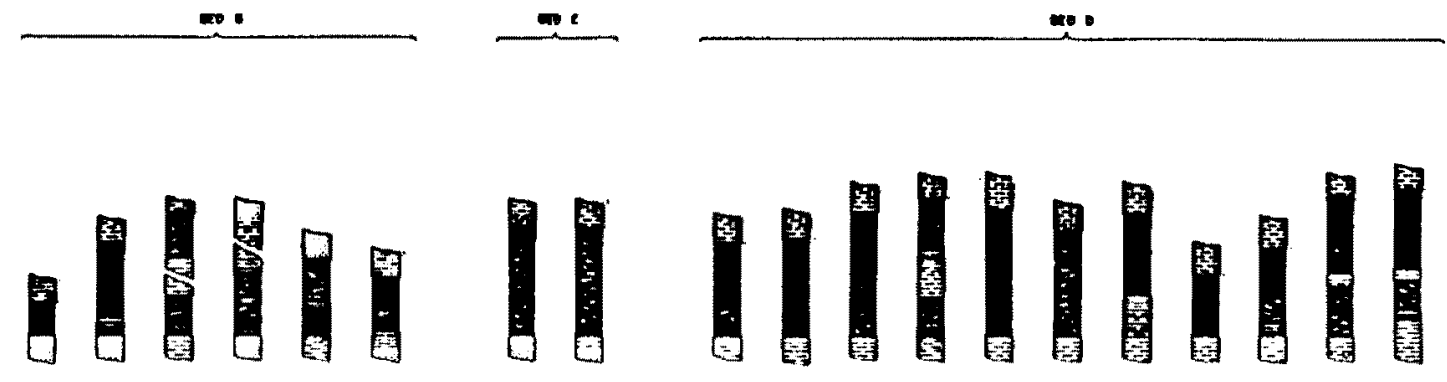

$\cos 2 \cos$

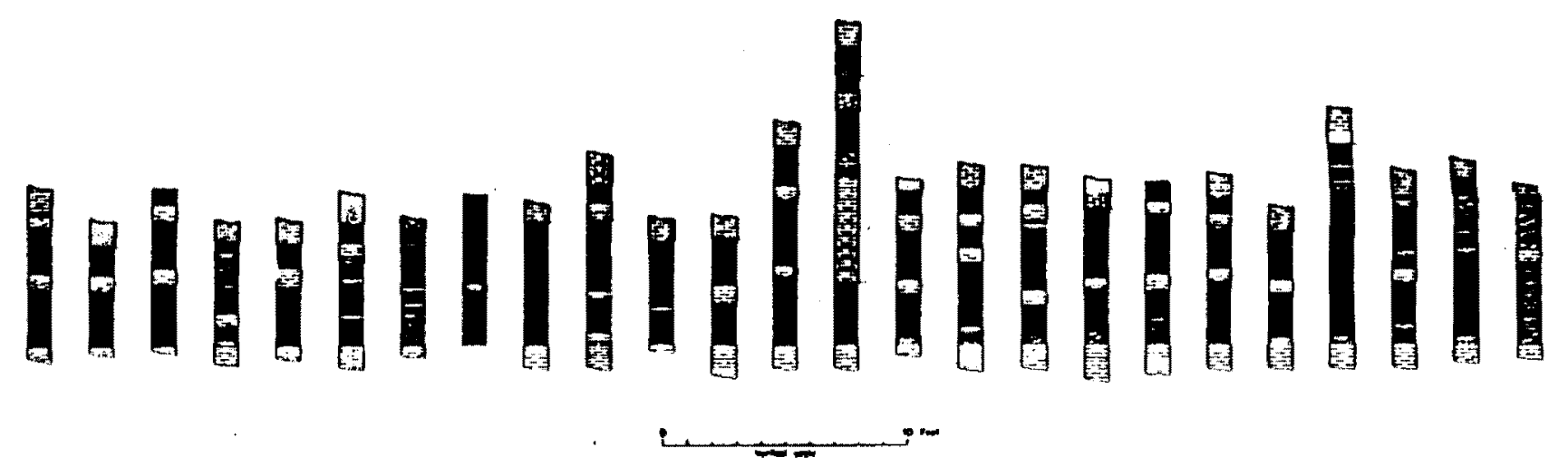

Figure 9. Variability and similarity among correlative coal seams, Coos Bay coal field. 
resulting profile of each of the major seams is distinctive, both in outcrop and on geophysical logs. The Beaver Hill seam, for example, is clearly identifiable in the coastal cliffs at Yokam Point, 22.5 kilometers (14 miles) from its exposure at Cedar Point in the Coaledo Basin (Robertson, 1982).

A compilation of the occurrences of the major coal seams in each of the logged drill-holes (Table I) makes it clear that they have not been subjected to appreciable post-depositional erosion. Among the minor seams, the stratigraphic record is nearly as complete. Only two instances of coal seam erosion ("wants") could be documented. In each of these cases, less than 7 meters $(21$ feet) of section had been removed and the section in adjacent drill-holes was normal, indicating that the erosion was localized in both time and space.

The general petrologic character of the lithostratigraphic units in the region and their first-order cyclicity were recognized by early workers (Diller, 1899; Diller and Pishel, 1909; Allen and Baldwin, 1944; Dott, 1966) and lead to an interpretation of the depositional environment involving alternating progradation and inundation of a deltaic complex of considerable extent (for an alternate hypothesis, refer to Duncan, 1953). This interpretation has been extended in more recent years by work in the excellent exposures along the coastal cliffs 
Table I. Occurrence of major coal seams in drill holes in the China Creek area.

\begin{tabular}{|c|c|c|c|c|c|c|c|c|c|c|c|c|c|c|c|}
\hline $\begin{array}{l}\text { ORILL HOLE } \\
\text { NUMBER }\end{array}$ & $\begin{array}{l}\text { LOCATION } \\
\text { (SEC/TOWN/RANGE) }\end{array}$ & $\begin{array}{l}\text { THICKNESS } \\
\text { PENETRRTED } \\
\text { (FEET) }\end{array}$ & $\begin{array}{l}\text { LOCAL OIP } \\
\text { (DEGREES) }\end{array}$ & $\begin{array}{c}\text { SURFACE } \\
\text { ELEVTYTION } \\
\text { (FET) }\end{array}$ & $\begin{array}{l}\text { ELEVATI ION } \\
\text { "N" SEAM } \\
\text { "TOP) }\end{array}$ & $\begin{array}{l}\text { ELEVATI ION } \\
\text { "JN" SERM } \\
\text { (BSE) }\end{array}$ & $\begin{array}{l}\text { ELEUATION } \\
\text { "EN SEAM } \\
\text { STOP }\end{array}$ & $\begin{array}{l}\text { ELEVATION } \\
\text { "DN SERM } \\
\text { (BAE) } \\
\end{array}$ & $\begin{array}{l}\text { ELEUAT ION } \\
\text { "BH" SEAM } \\
\text { (TOP) }\end{array}$ & $\begin{array}{l}\text { ELEVAT ION } \\
\text { "BH" SEAM } \\
\text { (BAE) }\end{array}$ & $\begin{array}{l}\text { ORILLED } \\
\text { THICKNESS } \\
\text { "Ju SEAM }\end{array}$ & 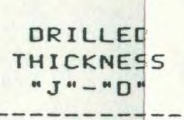 & $\begin{array}{l}\text { ORILLED } \\
\text { THICNEESS } \\
0 " \text { DEAM } \\
0 \text { SEAM }\end{array}$ & $\begin{array}{l}\text { ORILLED } \\
\text { THICKNESS } \\
\text { "HIN-BBH" }\end{array}$ & $\begin{array}{l}\text { ORILLED } \\
\text { THICNESSS } \\
\text { "BH" SEPMM }\end{array}$ \\
\hline $\begin{array}{l}\mathrm{CB}-01 \\
\mathrm{CB}-02\end{array}$ & 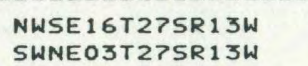 & $\begin{array}{r}1190 \\
820\end{array}$ & $\begin{array}{l}10 \\
20\end{array}$ & $\begin{array}{r}180 \\
5\end{array}$ & $\begin{array}{l}-994 \\
-384\end{array}$ & $\begin{array}{l}-952 \\
-394\end{array}$ & ${ }_{-570}^{<-5}$ & $-\quad$ NOT DR & ILLED -- & -704 & $\begin{array}{r}8 \\
10\end{array}$ & $\overline{176}$ & $\overline{6}$ & -120 & $-\overline{8}$ \\
\hline 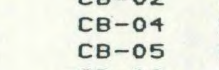 & 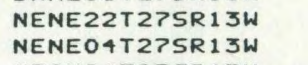 & $\begin{array}{l}1260 \\
1425\end{array}$ & $\begin{array}{l}10 \\
15\end{array}$ & $\begin{array}{r}\begin{array}{r}60 \\
40\end{array} \\
0\end{array}$ & $\begin{array}{l}-2 \\
-907\end{array}$ & $\begin{array}{r}-9 \\
-919\end{array}$ & $\begin{array}{l}-405 \\
-1283\end{array}$ & $\begin{array}{l}-410 \\
-1288\end{array}$ & $\begin{array}{l}-540 \\
<\text { NOT OR } \\
<\text { Not }\end{array}$ & ILLED $\begin{array}{c}-545 \\
?\end{array}$ & $\begin{array}{l}10 \\
12\end{array}$ & $\begin{array}{l}\begin{array}{l}176 \\
396 \\
364\end{array} \\
364\end{array}$ & $\begin{array}{l}5 \\
5 \\
5 \\
5\end{array}$ & $\begin{array}{l}120 \\
30 \\
-\end{array}$ & $\begin{array}{r}8 \\
-5 \\
--\end{array}$ \\
\hline 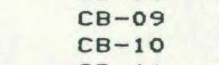 & 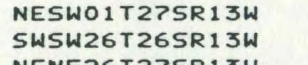 & $\begin{array}{l}1500 \\
1185 \\
1185\end{array}$ & $\begin{array}{l}10 \\
15\end{array}$ & $\begin{array}{l}40 \\
380\end{array}$ & $\begin{array}{l}-1241 \\
-274\end{array}$ & $\begin{array}{r}1423 \\
-286\end{array}$ & $\begin{array}{l}-1126 \\
-455\end{array}$ & $\begin{array}{l}-1460 \\
-458 \\
-457\end{array}$ & $\begin{array}{l}<\text { NOT DR } \\
-570 \\
\text { D }\end{array}$ & $\begin{aligned} \text { ILLED } & > \\
-576 & \end{aligned}$ & $\begin{array}{r}9 \\
12\end{array}$ & $\begin{array}{l}182 \\
169\end{array}$ & $\begin{array}{l}5 \\
5 \\
3\end{array}$ & 112 & $-\frac{1}{6}$ \\
\hline $\begin{array}{l}\mathrm{C}=11 \\
\mathrm{C}-11 \\
\mathrm{CB}-12\end{array}$ & 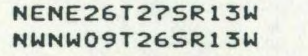 & $\begin{array}{r}\begin{array}{r}1195 \\
250\end{array}\end{array}$ & $\begin{array}{l}10 \\
10 \\
10\end{array}$ & $\begin{array}{l}\begin{array}{l}680 \\
420\end{array} \\
4\end{array}$ & ${ }_{346}^{218}$ & $\begin{array}{l}2088 \\
337\end{array}$ & & $\begin{array}{l}-197 \\
--N O T \text { OR }\end{array}$ & $\begin{array}{l}\text { ILLED - } 22 \\
\text { ILLE }\end{array}$ & $\begin{array}{l}-330 \\
-->\end{array}$ & $\begin{array}{c}10 \\
9\end{array}$ & 400 & 5 & 125 & 8 \\
\hline $\begin{array}{l}\mathrm{CB}-14 \\
\mathrm{CB}-17\end{array}$ & 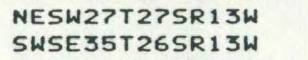 & $\begin{array}{l}1520 \\
1400\end{array}$ & $\begin{array}{l}15 \\
20\end{array}$ & $\begin{array}{r}85 \\
155\end{array}$ & $\begin{array}{l}-935 \\
-800\end{array}$ & $\begin{array}{l}-944 \\
-814\end{array}$ & $\begin{array}{c}-1272 \\
-997\end{array}$ & $\begin{array}{l}-1278 \\
-1003\end{array}$ & $\begin{array}{l}-1424 \\
-113\end{array}$ & $\begin{array}{l}-1433 \\
-1119\end{array}$ & ${ }_{14}^{9}$ & $\begin{array}{l}328 \\
183\end{array}$ & $\begin{array}{l}6 \\
6 \\
\end{array}$ & $\begin{array}{l}146 \\
110\end{array}$ & 9 \\
\hline $\begin{array}{l}\begin{array}{c}c B-20 \\
C B-21\end{array} \\
C B\end{array}$ & 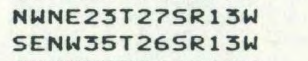 & $\begin{array}{r}700 \\
1240\end{array}$ & $\begin{array}{r}10 \\
5\end{array}$ & $\begin{array}{l}440 \\
260\end{array}$ & $\begin{array}{l}<\text { NOT DR1 } \\
-531\end{array}$ & $\begin{array}{l}\text { ILLED }>> \\
-542 \\
\text { S }\end{array}$ & $\begin{array}{c}200 \\
-688\end{array}$ & $\begin{array}{l}203 \\
-691\end{array}$ & $\begin{array}{r}84 \\
-793\end{array}$ & $\begin{array}{r}75 \\
-798 \\
\end{array}$ & $\overline{11}$ & 146 & $\begin{array}{l}6 \\
3\end{array}$ & $\begin{array}{l}119 \\
102\end{array}$ & $\begin{array}{l}9 \\
5\end{array}$ \\
\hline $\begin{array}{c}c B-22 \\
C B-23\end{array}$ & 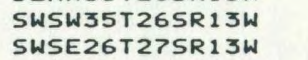 & $\begin{array}{l}1357 \\
1360\end{array}$ & $\begin{array}{l}15 \\
15\end{array}$ & $\begin{array}{l}155 \\
610\end{array}$ & $\begin{array}{l}-629 \\
-28\end{array}$ & $\begin{array}{l}-643 \\
-38\end{array}$ & $\begin{array}{l}-810 \\
-431\end{array}$ & $\begin{array}{l}-885 \\
-434\end{array}$ & $\begin{array}{l}-928 \\
-554\end{array}$ & $\begin{array}{l}-934 \\
-562\end{array}$ & $\begin{array}{l}14 \\
10\end{array}$ & $\begin{array}{l}167 \\
393\end{array}$ & $5_{3}^{5}$ & $\begin{array}{l}113 \\
120\end{array}$ & $\begin{array}{l}6 \\
8 \\
8\end{array}$ \\
\hline $\begin{array}{l}c B-25 \\
c 8-26 \\
c 8\end{array}$ & 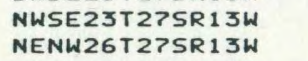 & $\begin{array}{r}925 \\
1497\end{array}$ & $\begin{array}{l}10 \\
10\end{array}$ & $\begin{array}{l}520 \\
590\end{array}$ & $\begin{array}{r}245 \\
-382\end{array}$ & $\begin{array}{r}235 \\
-384\end{array}$ & $\begin{array}{l}-105 \\
-799\end{array}$ & $\begin{array}{l}-111 \\
-804\end{array}$ & $\begin{array}{l}-243 \\
-926\end{array}$ & $\begin{array}{l}-251 \\
-938\end{array}$ & $\begin{array}{c}10 \\
2 \\
2\end{array}$ & $\begin{array}{l}340 \\
415\end{array}$ & $\begin{array}{l}6 \\
5 \\
5\end{array}$ & $\begin{array}{l}132 \\
122\end{array}$ & $\begin{array}{r}8 \\
12\end{array}$ \\
\hline 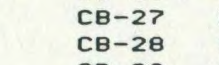 & 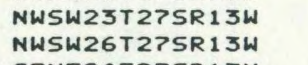 & $\begin{array}{l}1357 \\
1500\end{array}$ & $\begin{array}{l}10 \\
10 \\
10\end{array}$ & $\begin{array}{l}470 \\
140 \\
140\end{array}$ & $\begin{array}{l}-89 \\
-747\end{array}$ & $\begin{array}{l}-99 \\
-756\end{array}$ & $\begin{array}{l}-474 \\
-1170\end{array}$ & $\begin{array}{l}-480 \\
-1176 \\
-176\end{array}$ & $\begin{array}{r}-597 \\
-1301\end{array}$ & $\begin{array}{l}-6022 \\
-1309\end{array}$ & $\begin{array}{r}10 \\
9 \\
9\end{array}$ & $\begin{array}{l}375 \\
414\end{array}$ & $\begin{array}{l}6 \\
6\end{array}$ & $\begin{array}{l}117 \\
125 \\
125\end{array}$ & $\begin{array}{r}5 \\
5 \\
8\end{array}$ \\
\hline $\begin{array}{l}\begin{array}{l}c 8-29 \\
c B-30\end{array} \\
C B-3\end{array}$ & 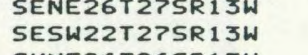 & $\begin{array}{r}719 \\
1500\end{array}$ & $\begin{array}{l}15 \\
10\end{array}$ & $\begin{array}{l}580 \\
300\end{array}$ & $\begin{array}{l}69 \\
-565 \\
-565\end{array}$ & $\begin{array}{r}58 \\
-572 \\
-572\end{array}$ & -1012 & $\begin{array}{l}-1 \text { NOT } \\
-1017\end{array}$ & 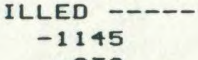 & -1154 & $\begin{array}{c}11 \\
7\end{array}$ & 440 & 5 & 128 & $-\overline{9}$ \\
\hline 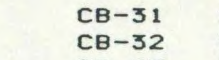 & 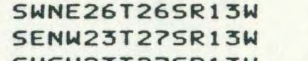 & $\begin{array}{r}1299 \\
841\end{array}$ & $\begin{array}{l}20 \\
10\end{array}$ & $\begin{array}{l}280 \\
420\end{array}$ & $\begin{array}{r}-656 \\
525\end{array}$ & $\begin{array}{r}-667 \\
242 \\
\end{array}$ & $\begin{array}{l}-813 \\
-11 \%\end{array}$ & $\begin{array}{l}-818 \\
-120\end{array}$ & $\begin{array}{l}-9939 \\
-248\end{array}$ & $\begin{array}{l}-944 \\
-259\end{array}$ & $\begin{array}{l}11 \\
10 \\
10\end{array}$ & $\begin{array}{l}146 \\
356\end{array}$ & $\begin{array}{c}5 \\
6 \\
6\end{array}$ & $\begin{array}{l}121 \\
128\end{array}$ & $\begin{array}{r}5 \\
11\end{array}$ \\
\hline $\begin{array}{l}C_{M F-33}=-33 \\
M F-01\end{array}$ & 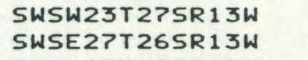 & $\begin{array}{l}1340 \\
1500\end{array}$ & $\begin{array}{l}10 \\
20\end{array}$ & $\begin{array}{l}250 \\
100\end{array}$ & $\begin{array}{l}-189 \\
<--- \\
\end{array}$ & $\begin{array}{l}-196 \\
-\quad \text { NOT DR }\end{array}$ & ILLEO -62.3 & $\begin{array}{l}-630 \\
--\rightarrow>\end{array}$ & $\begin{array}{r}-745 \\
49\end{array}$ & $\begin{array}{r}-754 \\
42\end{array}$ & $\therefore$ & 427 & $?$ & $\begin{array}{l}115 \\
---\end{array}$ & $?$ \\
\hline $\begin{array}{l}M F-22 \\
M F-03\end{array}$ & 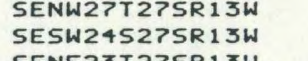 & $\begin{array}{l}\begin{array}{l}1165 \\
1501\end{array} \\
501\end{array}$ & 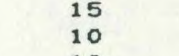 & $\begin{array}{r}40 \\
350\end{array}$ & 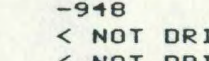 & $\begin{array}{l}\text { ILLED }>\text { - } \\
\text { IST }\end{array}$ & 222 & $\begin{array}{l}\text { NOT DR } \\
216\end{array}$ & ILLED - & $\rightarrow-385$ & -9 & --- & $\overline{6}$ & 108 & 10 \\
\hline $\begin{array}{l}\text { MFF-06 } \\
\text { MF-or }\end{array}$ & 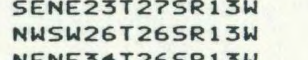 & $\begin{array}{l}561 \\
699 \\
690\end{array}$ & $\begin{array}{l}10 \\
15\end{array}$ & $\begin{array}{l}400 \\
40 \\
40\end{array}$ & 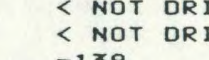 & $\begin{array}{l}\text { ITLED ? } \\
\text { ILLED }\end{array}$ & $\begin{aligned}-90 \\
-33 \\
-33\end{aligned}$ & $\begin{array}{r}85 \\
-38 \\
-38\end{array}$ & $\begin{array}{l}-28 \\
-157 \\
-157\end{array}$ & $\begin{array}{l}-35 \\
-165 \\
-165\end{array}$ & $\overline{-z}$ & $-\overline{-}$ & $\begin{array}{c}5 \\
5 \\
5\end{array}$ & $\begin{array}{l}\begin{array}{l}113 \\
119\end{array} \\
119\end{array}$ & 8 \\
\hline $\begin{array}{l}\mathrm{MF}-09 \\
\mathrm{MF}-1 \mathrm{0}\end{array}$ & 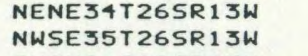 & $\begin{array}{c}559 \\
1434\end{array}$ & $\begin{array}{l}15 \\
20\end{array}$ & $\begin{array}{l}110 \\
240\end{array}$ & $\begin{array}{l}-1399 \\
-775\end{array}$ & $\begin{array}{l}-153 \\
-790\end{array}$ & $\begin{array}{l}-274 \\
-969\end{array}$ & $\begin{array}{l}-278 \\
-974\end{array}$ & -1081 & $\begin{array}{l}-\begin{array}{l}-04 \\
-1087\end{array} \\
\end{array}$ & $\begin{array}{l}14 \\
15\end{array}$ & $\begin{array}{l}\begin{array}{l}121 \\
179\end{array} \\
179\end{array}$ & $\begin{array}{l}4 \\
5\end{array}$ & $\begin{array}{l}\begin{array}{l}119 \\
107\end{array} \\
107\end{array}$ & $\begin{array}{l}7 \\
6\end{array}$ \\
\hline $\begin{array}{l}M F-11 \\
65-03 \\
M\end{array}$ & 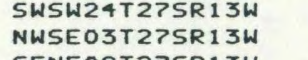 & $\begin{array}{r}501 \\
1175\end{array}$ & 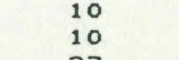 & $\begin{array}{r}410 \\
5\end{array}$ & $\begin{array}{l}<\text { NOT DRI } \\
-240\end{array}$ & $\begin{array}{l}\text { ILLED } 3 \\
-243 \\
L_{3}\end{array}$ & $\begin{array}{l}69 \\
-585\end{array}$ & $\begin{array}{l}-593 \\
-590\end{array}$ & $\begin{array}{l}-49 \\
-715\end{array}$ & $\begin{array}{l}-56 \\
-723 \\
\end{array}$ & 3 & 342 & $\begin{array}{c}6 \\
5 \\
5\end{array}$ & 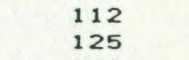 & $\begin{array}{l}7 \\
8\end{array}$ \\
\hline $\begin{array}{l}\text { S5 } \\
65-05 \\
\text { S5-05 }\end{array}$ & 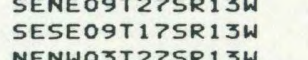 & $\begin{array}{l}704 \\
725\end{array}$ & $\begin{array}{l}27 \\
27 \\
37\end{array}$ & $\begin{array}{l}23 \\
31 \\
3\end{array}$ & $\begin{array}{l}<\text { NOT } \\
<\text { NOT } \\
\text { NOR }\end{array}$ & $\begin{array}{l}\text { YILED } \\
\text { IILED ? }\end{array}$ & $\begin{array}{l}-\begin{array}{l}-475 \\
-458\end{array} \\
-450\end{array}$ & $\begin{array}{l}-450 \\
-463 \\
-466\end{array}$ & $\begin{array}{l}-660 \\
-674 \\
-278\end{array}$ & $\begin{array}{l}-667 \\
-682 \\
-274\end{array}$ & $\overline{--}$ & $\overline{---}$ & $\begin{array}{c}5 \\
5 \\
5\end{array}$ & $\begin{array}{l}210 \\
211\end{array}$ & $\begin{array}{l}7 \\
8\end{array}$ \\
\hline $\begin{array}{l}65-06 \\
65-07 \\
65-08\end{array}$ & 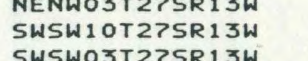 & $\begin{array}{r}416 \\
1310 \\
570\end{array}$ & ? & $\begin{array}{l}20^{2} \\
20\end{array}$ & $\begin{array}{l}-857 \\
-857 \\
-251\end{array}$ & $\begin{array}{l}\text { ILE } \\
-860 \\
-867\end{array}$ & $\begin{array}{c}<-92 \\
<-2 . \\
\end{array}$ & $\begin{array}{l}-966 \text { OR } \\
-\quad \text { NOT }\end{array}$ & 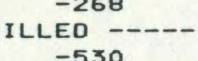 & & $-\frac{1}{3}$ & & 4 & & -6 \\
\hline $\begin{array}{l}\text { 65-08 } \\
\text { 65-09 }\end{array}$ & 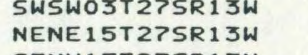 & 808 & $\begin{array}{l}20 \\
24\end{array}$ & $?$ & 年 & $\begin{array}{l}-294 \\
-294\end{array}$ & 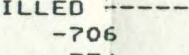 & -712 & $\begin{array}{l}-530 \\
<\text { NOT OR }\end{array}$ & $\begin{array}{l}-534 \\
\text { RILLED }\end{array}$ & 10 & 412 & $\frac{-}{6}$ & --- & -4 \\
\hline $\begin{array}{l}\text { 65-10 } \\
\text { GS-11 }\end{array}$ & 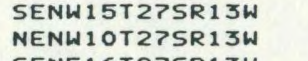 & & $\begin{array}{l}24 \\
20\end{array}$ & $249^{4}$ & $\begin{array}{l}-438 \\
-595 \\
-500\end{array}$ & $\begin{array}{l}-448 \\
-602\end{array}$ & $\begin{array}{l}-\begin{array}{l}-754 \\
-985\end{array} \\
-985\end{array}$ & $\begin{array}{l}-759 \\
-990\end{array}$ & $\begin{array}{l}<\text { NOT DR } \\
-1133\end{array}$ & & $\begin{array}{c}10 \\
?\end{array}$ & $\begin{array}{l}\begin{array}{l}306 \\
383\end{array} \\
383\end{array}$ & $\begin{array}{c}5 \\
5\end{array}$ & 147 & 7 \\
\hline $\begin{array}{l}\text { 65-12 } \\
\text { GS-13 }\end{array}$ & 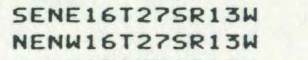 & $\begin{array}{l}1574 \\
1174\end{array}$ & 28 & $\begin{array}{l}112 \\
315\end{array}$ & $\begin{array}{l}-708 \\
-137\end{array}$ & $\begin{array}{l}-716 \\
-143\end{array}$ & 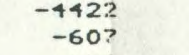 & $\begin{array}{r}-1126 \\
-610\end{array}$ & $\begin{array}{r}-1313 \\
-839 \\
-839\end{array}$ & $\begin{array}{r}-1318 \\
-849\end{array}$ & $\begin{array}{l}8 \\
6\end{array}$ & $\begin{array}{l}406 \\
464 \\
464\end{array}$ & $\frac{4}{3}$ & $\begin{array}{l}187 \\
229\end{array}$ & $\begin{array}{r}5 \\
10 \\
\end{array}$ \\
\hline $\begin{array}{l}65-14 \\
\text { GS-15 }\end{array}$ & 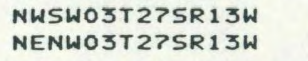 & $\begin{array}{l}655 \\
564\end{array}$ & $\begin{array}{l}19 \\
16\end{array}$ & $\begin{array}{c}134 \\
73\end{array}$ & 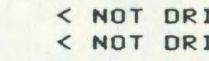 & $\begin{array}{l}\text { ILLED > } \\
\text { ILLED }>\end{array}$ & $\begin{array}{l}-132 \\
-136\end{array}$ & $\begin{array}{l}-137 \\
-140\end{array}$ & $\begin{array}{l}-256 \\
-279\end{array}$ & $\begin{array}{l}-262 \\
-286\end{array}$ & -- & --- & $\begin{array}{c}5 \\
4 \\
\end{array}$ & $\begin{array}{l}119 \\
130\end{array}$ & $\frac{6}{7}$ \\
\hline
\end{tabular}


between Coos Bay and Bandon (Rooth, 1974; Ryberg, 1978; Chan, 1982). Each of these workers suggested that the cyclic progradation and inundation of the Coaledo delta complex may have been related to its position on an active tectonic margin. The composition of the framework grains has also been investigated (Dott, 1966, Heller and Ryberg, 1983) and lead to the suggestion that the fluvial system which built the delta complex had its origin on the western slopes of the Cordillera but was subjected to considerable influence by the developing Cascade volcanic arc. The first-order stratigraphic observations and petrologic analyses made during this study are consistent with these interpretations.

\section{STRUCTURAL GEOLOGY}

The first-order structure of the study area, as interpreted from the surface and subsurface information described above, is summarized in Figure 10. The major structural feature is a broad, open, northeasterly plunging structural basin, referred to here as the Coaledo Basin. The central portion of this structure is bounded on the southeast by a northeast-trending anticline, the Pulaski Arch, which separates it from a smaller basin to the southeast, known as the Coquille Basin. The Pulaski Arch loses its identity northeast of the town of Coquille, where 
BEDROCK GEOLOGY

OF THE COOS BAY COAL FIELD

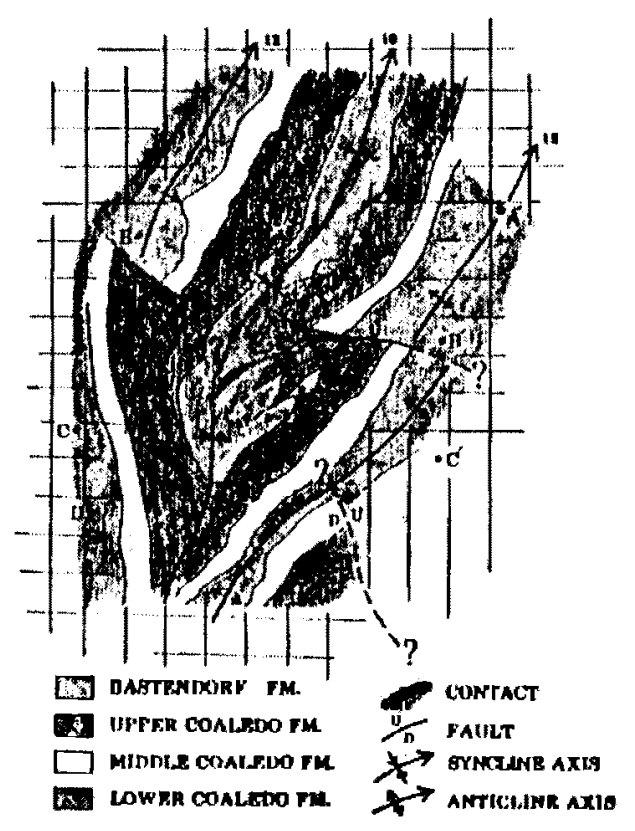

SEQUENTIAL CROSS-SECTIONS, COOS BAY COAL FIELD

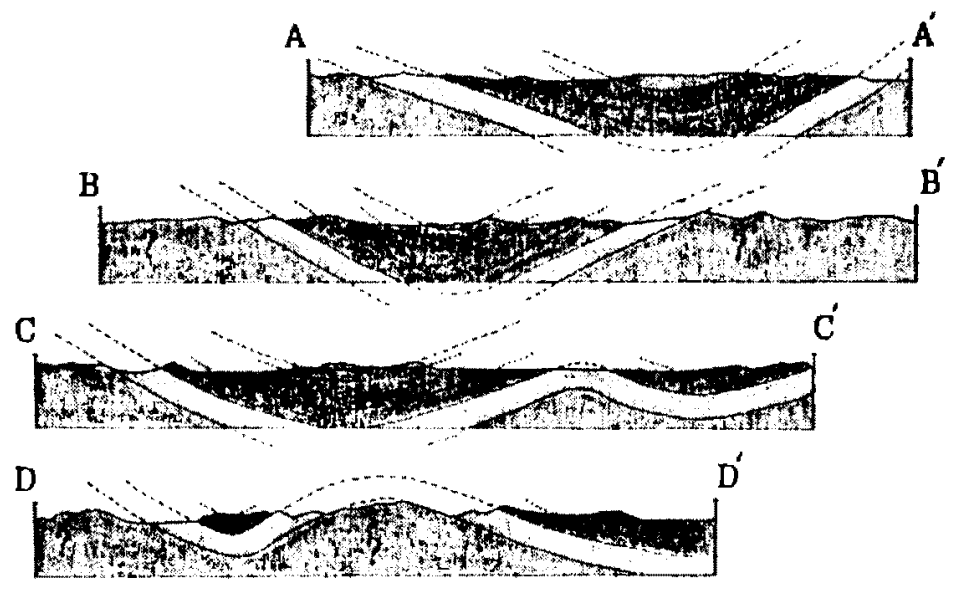

Fiqure 10. Generalized geologic map and sequential cross-sections, Coos Bay coal field. 
the two basins merge. The northeastern boundary of the resulting structure is truncated by erosion and a series of large outcrops of the Roseburg Volcanics, which separate the Coaledo Basin from the much larger Tyee Basin to the east. The northwestern flank of the Coaledo Basin is bounded by the Westport Arch, a broad, open anticline trending to the northeast which separates the Coaledo Basin from the much smaller South slough Basin to the northwest. On its southwestern margin, the Beaver slough Basin merges with the South Slough Basin, both being separated from the pre-Tertiary terrain to the southwest by the Cape Arago Anticline. Within the Coaledo Basin, several smaller scale folds occur, most notable among these is the Catching slough Arch, a small, elongate, northeasterly trending dome immediately west of the town of sumner.

Folding in the area deviates substantially from the ideal cylindrical form, the axial surfaces are nonplanar and the profiles of the folds are asymmetrical, the eastern limbs being steeper than their western counterparts. The folding style, however, is very nearly concentric, there being no detectable, systematic thickening of the folded strata in the hinge areas of the folds or thinning on the limbs. The overall structural pattern is a radiating series of north to northeasterly plunging folds, which diverge, flatten, lose amplitude and increase in wavelength towards the northeast. 
A broad, first-order fault system trends in a northwesterly direction across the central portion of the Coaledo Basin, dividing the study area into three distinct structural domains. The northeastern portion of the area has been uplifted approximately 600 meters $(2,000$ feet) across this fault zone relative to the southwestern portion and approximately 1.5 kilometers $(5,000$ feet) of left-lateral strike-slip displacement has occurred across the fault zone. A series of smaller, high-angle, dip-slip faults occupies the central portion of the study area between splays of the major fault system. Movement across these faults, which occur in an anastomosed pattern trending across the major fault zone, is an order of magnitude smaller than that of the major fault system and is down to the southeast in every case.

The nearly uniform thickness of the major lithostratigraphic units participating in the first-order folding is an indication that the deformation occurred after the youngest of the units exposed in the area was deposited. Additional evidence of the post-depositional nature of the folding comes from a comparison of the attitudes of primary bedding in different portions of the basin (figure 11). No systematic relationship between location and the degree or style of deformation of the units is evident, as would be expected if the structural basin had been forming as deposition proceeded. The fault along the axis of the 


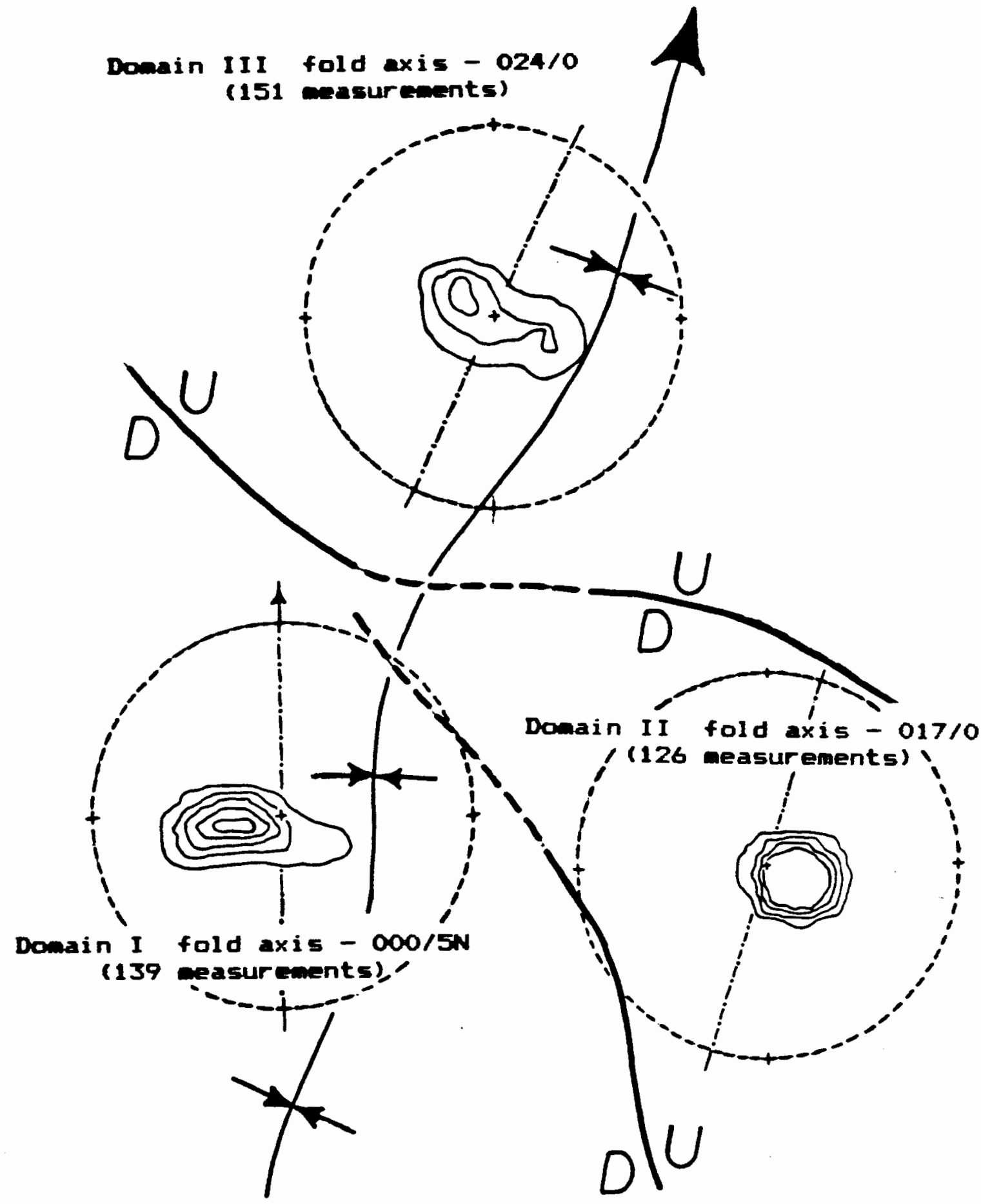

Figure 11. Differential fold analysis for three major structural domains, Coaledo coal field. 
Pulaski Arch probably occurred during the folding event. It separates the Beaver Slough Basin from the adjacent Coquille Basin only in the southeastern portion of the study area where the axes of the two structures approach one another and the radius of flexure of the Pulaski Arch begins to decrease sharply. The fault is not identifiable northeast of the Coquille River, where the Pulaski Arch opens into a broader structure.

The major fault zone crossing the basin from southeast to northwest in the central portion of the area, referred to here as the Transverse Fault Zone, appears to have been the result of a second episode of deformation. The axis of the basin has been offset by approximately 1.5 kilometers $(5,000$ feet $)$ across this fault zone in a left-lateral sense and the trace of the axis shows indications of drag in the vicinity of the fault zone. Moreover, there is approximately 600 meters $(2,000$ feet) of net slip across the fault zone, the northeastern portion of the area having been uplifted relative to the southwestern portion. The outcrop pattern of the folded strata are sharply truncated by the fault zone and the sense of motion across the fault is in opposite directions on opposite sides of the basin axis, a situation which would be difficult to explain unless the slip across the fault zone postdated the folding. Finally, there is no discernable change in the style of the folding across this fault zone, as might be 
expected if the faulting and folding had occurred simultaneously.

The Transverse Fault Zone can be divided into three segments which merge at the approximate intersection of the fault zone and the axis of the Coaledo Basin. Northwest of the junction, the fault appears to be a single entity, referred to here as the Cox Canyon Fault, across which both dip-slip (up to the northeast) and strike-slip (left-lateral) motion have occurred. Southeast of the junction, the situation is more complex. The fault zone there appears to have split into two major faults, referred to as the Beaver Creek and Coquille River Faults, which diverge towards the southeast. The Beaver Creek Fault is very nearly co-linear with the Cox Canyon Fault and has experienced the same amount aild sense of dip-slip motion. The Coquille River Fault, however, appears to have experienced dip-slip motion which is not only much smaller, but is also in the opposite sense. The wedge-shaped block between these segments of the Transverse Fault zone is broken by a closely spaced series of high-angle normal faults across which the motion has invariably been down to the southeast. These faults are sub-parallel, anastomosed and form a recurved, serif-shaped pattern which is truncated by the Beaver Creek and Coquille River faults.

The location, orientation and estimated motion across and within the Transverse Fault Zone suggests the operation 
of a mechanism similar to that described by Crowell (1974) in southern California. There, double bends and splays interrupting the continuity of regional strike-slip faults have produced a wide variety of secondary vertical deformations, ranging from pull-apart basins to tipped fault-wedge blocks. Crowell interpreted these vertical deformations to be a direct result of the opening of zones of extension in the releasing bends of strike-lip faults. If the Transverse Fault zone is viewed as a splayed fault with a left-lateral strike-slip component, then the northeast-trending anastomosed pattern of high-angle normal faults in the inter-splay area can be explained as the result of the subsidence and tipping of a wedge into the extensional zone formed as strike-slip motion alternated between the splays (Figure 12). This interpretation is made particularly attractive by three features of the inter-splay zone, the recurved, serif-like pattern of the inter-splay faults, which suggests drag consistent with significant left-lateral motion across the fault zone, the consistent down-to-the-southeast normal motion across the faults, which suggests an overall extensional regime and the sharp boundaries of that extensional regime which coincide with the transverse fault segments.

The structural history of the Coaledo Basin appears to have been two-fold, consisting of an early phase during which the pervasive north to northeast-trending basins and 
UIEW ALONG THE TRANSUERSE FAULT ZONE (SE-NW)

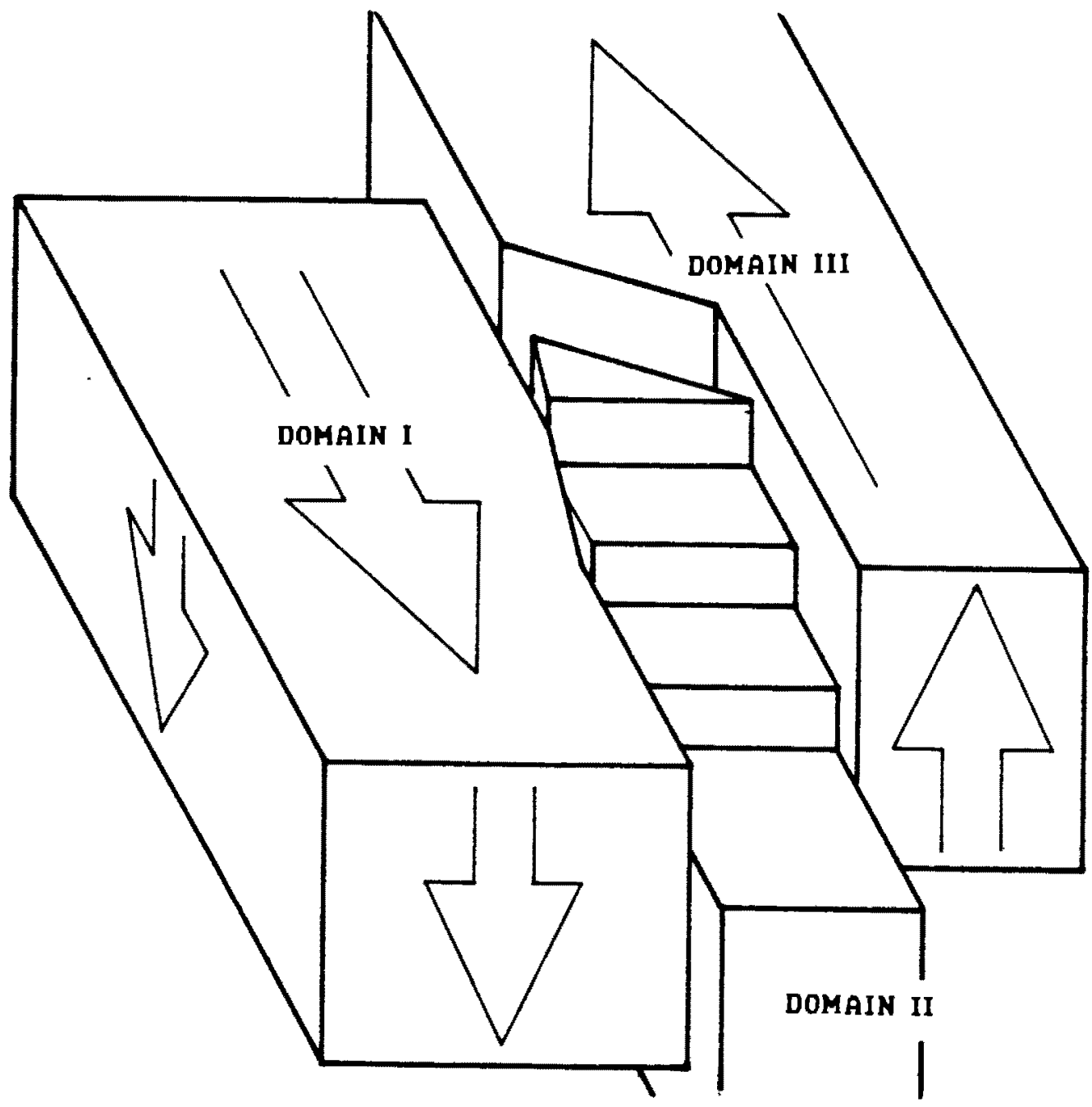

Figure 12. Structural model of faulting within the Coaledo Basin. 
arches were formed followed by a later phase during which the northwest-trending fault zone developed. The timing of these events is clear only to the extent that one stage followed the other and that both occurred after the deposition of the stratigraphic units exposed within the area had been completed. The regional stresses responsible for the folding episode were clearly compressional, with the axis of maximum stress nearly horizontal and varying in azimuth from approximately east-west to approximately northwest-southeast. The gentle, open style of the folding indicates that the stresses involved were below the elastic limit of the strata during the deformational event. In contrast, the second period of deformation appears to have been primarily torsional and evidently proceeded above the elastic limit of the strata.

\section{CONCLUSTONS}

The conclusions drawn from the preliminary stratigraphic and structural study of the Coaledo Basin are:

1) that deposition of the stratigraphic section in the basin was neither interrupted by significant erosional periods nor accompanied by significant deformation,

2) that the major coal seams in the upper member of the Coaledo Formation are distinctive, recognizable and of sufficient lateral extent to serve as useful stratigraphic 
markers,

3) that the textural diversity of the clastic rocks in the upper member of the Coaledo Formation is sufficient to serve as the basis of a lithological classification scheme. 
CHAPTER III

QUANTITATIVE STRATIGRAPHY

The area chosen for quantitative stratigraphic analysis is shown in Figure 13. Truncated on its northern border by the Transverse Eault Zone, on its southern and western borders by the coquille River Fault and on its eastern boundary by westerly-dipping outcrops of the marine shales of the middle member of the Coaledo Formation, the area occupies the central portion of the Coos Bay coalfield and the hinge of the coaledo Basin. The stratigraphic section includes portions of the middle and upper members of the Coaledo Formation and the basal portion of the Bastendorf Eormation. Geophysical logs of nineteen drill holes within the area permit examination of approximately 200 meters (600 feet) of the central, coal-bearing portion of the upper member of the Coaledo Formation. The distribution of drill holes allows resolution of lateral variations on the order of 0.8 kilometers $(0.5$ miles $)$. Vertical resolution, limited by the techniques used to record and analyze the geophysical logs and the contrast between adjacent lithological units, is on the order of a 


\section{GEOLOGIC MAP, CHINA CREEX}

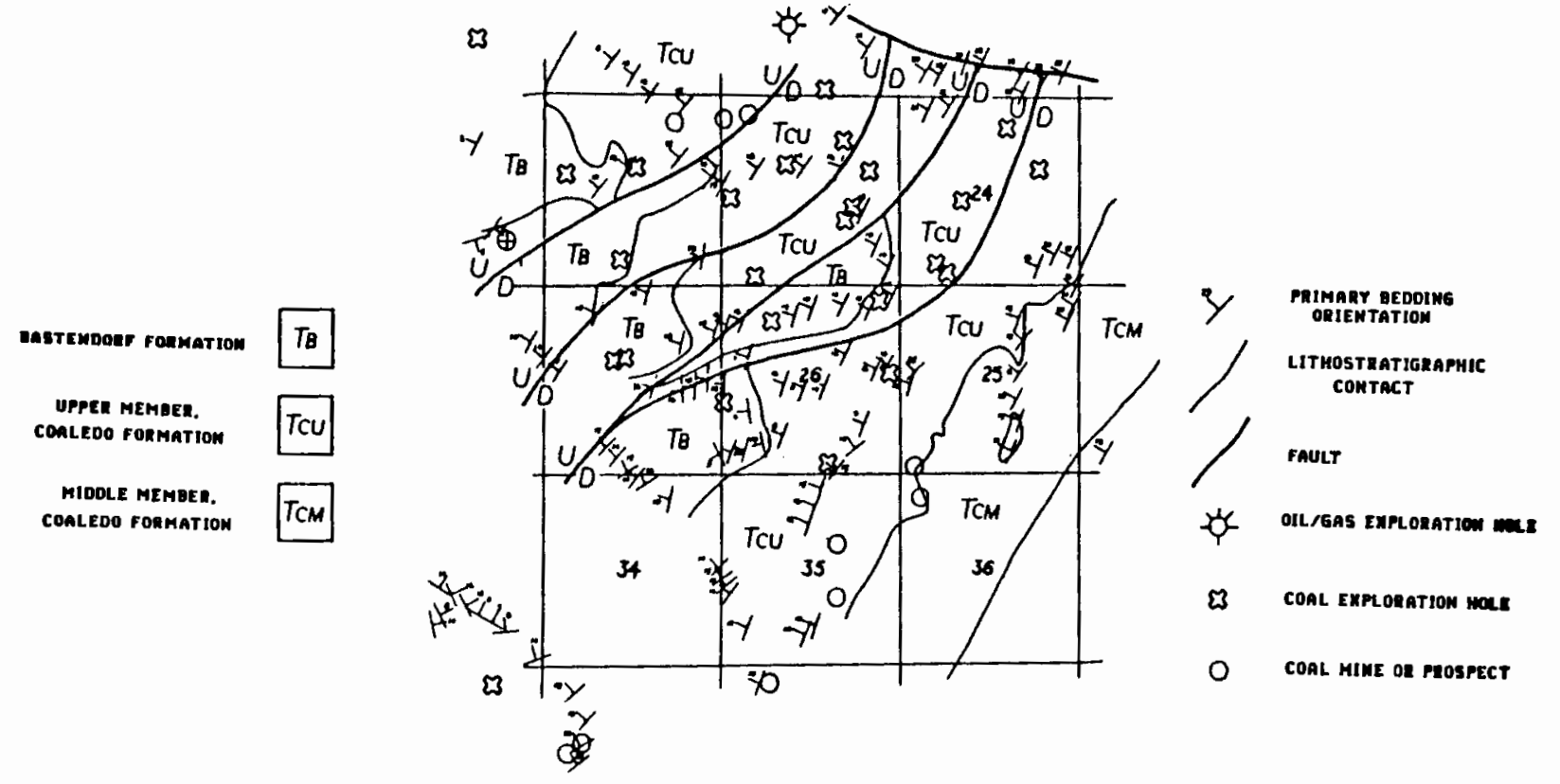

Figure 13. Generalized geologic map, China Creek area. 
meter ( 3 feet).

GEOPHYSICAL LOG INTERPRETATION

The analysis is based primarily on interpretations of geophysical log responses. Those lithological properties which generate recognizable log responses include thickness, texture, composition and density. Other characteristics such as primary sedimentary structures, internal fabrics, and fossil content have not been addressed. The information available for the drill holes consists of a mud log, cuttings, a core log and a geophysical log of the completed drill hole. A limited number of cores and cuttings were collected during the drilling program. Examination of these samples was carried out to test the interpretations derived from the analysis of the geophysical logs.

The suite of geophysical logs consists of simultaneous and continuous measurements of hole diameter (caliper), conventional resistivity, natural gamma emission (gamma) and induced gamma attenuation (density). A multi-step process was developed to interpret the geophysical logs. The initial step involves standardization of the response scales and digitization of the standardized log responses. This was done manually, the data being read from the logs as the mean response level for each log trace over 0.3 meter ( 1 foot) increments of depth. Due to 
differences between individual logging tools and variations in the composition of the drilling mud from hole to hole, calibration of the log responses was also necessary. A frequency response curve was constructed from the log responses in a reference drill hole (MF-09) and a linear correction factor was applied to the responses of each of the other drill holes in order to bring their peak response frequency into correspondence with that of the reference log (Figure 14). The resulting data represent the geophysical responses of the strata, measured in standardized and calibrated units.

The second step involves estimation of the bulk lithological properties of the strata from the geophysical responses which they generated. A number of methods for the determination of lithological properties from geophysical logs have been developed (Wyllie, 1963; Anonymous, 1972; Anonymous, 1982). Each involves the transformation of individual log responses into an unordered interval scale, the classes of which represent mutually exclusive lithologies. They differ from one another mainly in the types of geophysical logs on which the scales are based and the type of lithologies for which classes are defined. The two systems in common use in coal-bearing sedimentary sequences involve the use of natural gamma or resistivity responses for identification of clastic rocks and the induced gamma attenuation for recognition of carbon-rich 
DRILL HOLES MF-09\& CB-25

BEFORE RESISTIVITY CALIBRATION

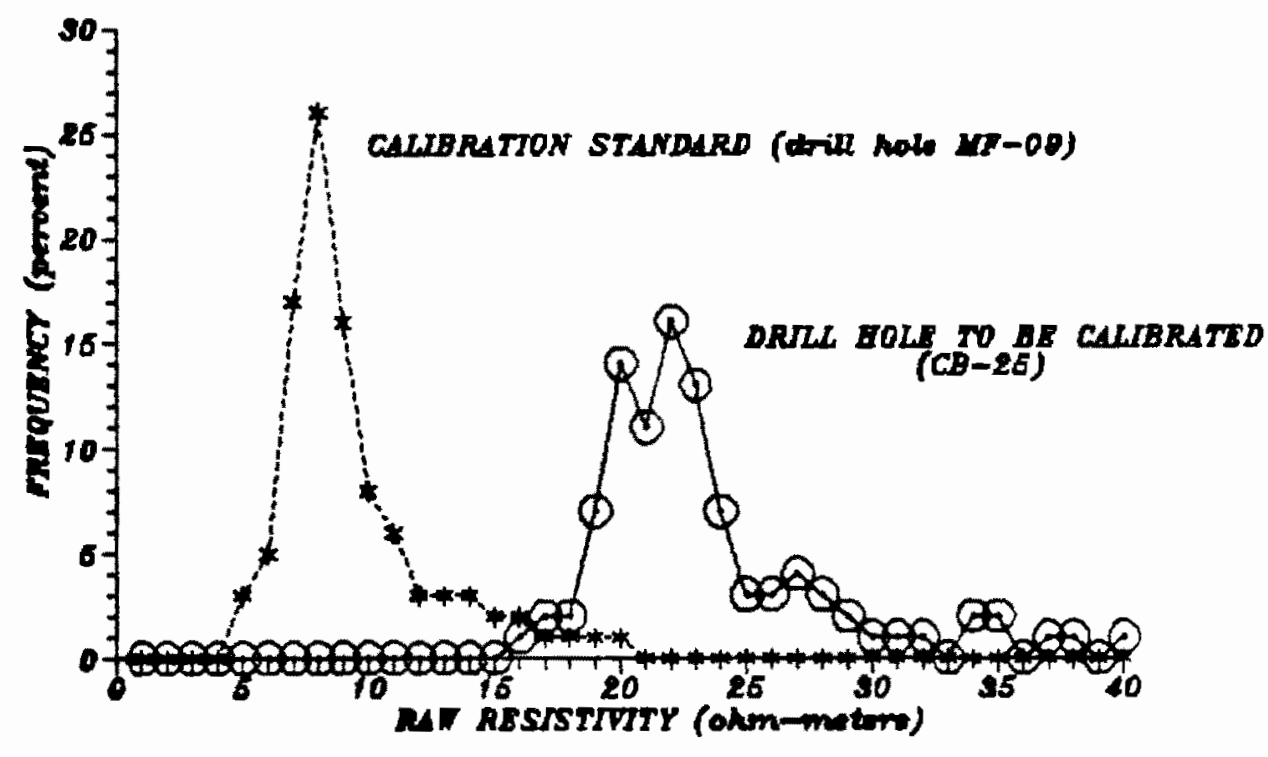

DRILL HOLES MF-O9 \& CB-25 AFTER RESISTIVITY CALIBRATION

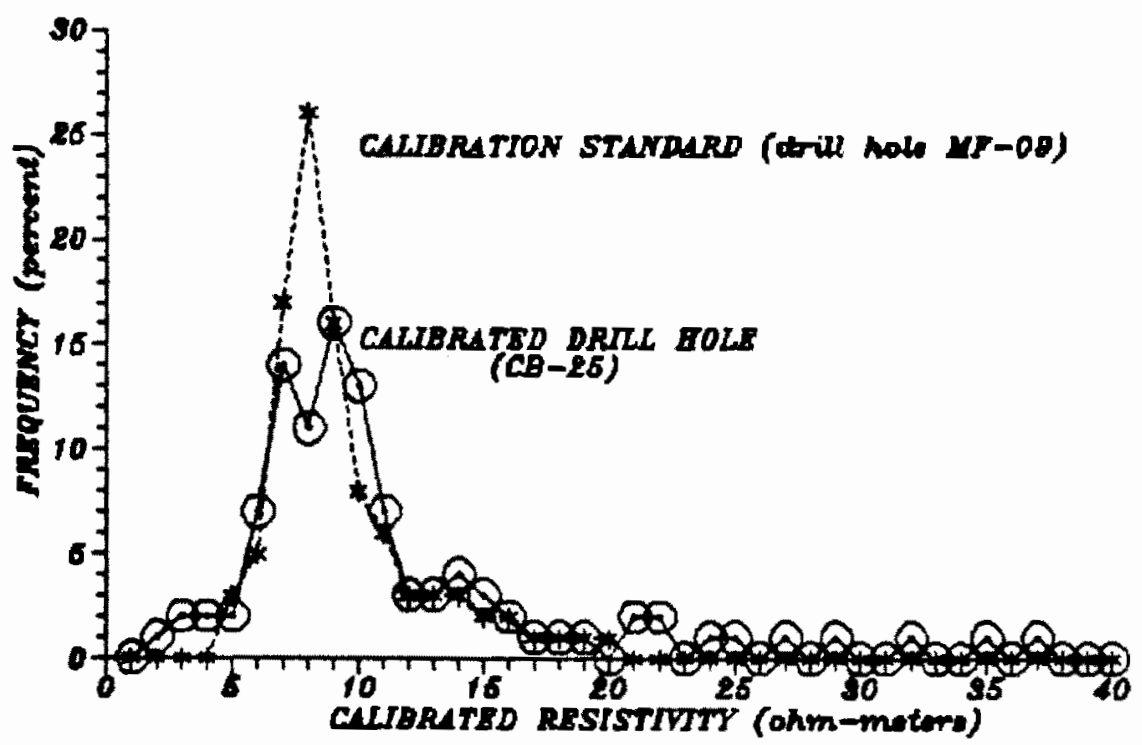

Figure 14. Calibration of a geophysical log. 
rocks (Vaninetti, 1981). Initial attempts to interpret the China Creek logs by these methods were made, but correspondence between the results of the two methods was found only in the distinction between the clastic and carbon-rich rocks. A comparison with lithologic descriptions of the logged holes indicated that neither the natural gamma nor the resistivity logs, used alone, was effective in identifying textural properties.

In order to improve the correlation between the lithologies inferred from the geophysical data and those obtained from direct examination of cuttings and cores, an alternative method of interpreting the geophysical data was developed. The method involves three steps:

1) An initial comparison of the caliper $\log$ and the drill bit diameter is made. If the hole diameter varies more than 3 inches from the diameter of the drill bit, then the lithology at that depth is considered to be indeterminate. This eliminates unreliable data caused by collapse or caving of the drill hole.

2) The carbon-rich rocks, clay-rich rocks and coarse clastic rocks are then separated, using the induced gamma attenuation and natural gamma emission responses. Those rocks whose density, as indicated by the induced gamma attenuation response, is less than $2.0 \mathrm{gm} / \mathrm{cc}$ are considered to be coal, densities between 2.0 and $2.2 \mathrm{gm} / \mathrm{cc}$ are considered to represent carbonaceous shale, and densities 
greater than $2.2 \mathrm{gm} / \mathrm{cc}$ are considered to indicate clastic rocks. Of the rocks in the clastic category, those whose natural gamma response is greater than 100 API units are interpreted as claystones, the remainder are interpreted as coarse clastic rocks. This initial classification is based on geophysical responses which are largely controlled by chemical composition (Vaninetti, 1981).

3) Aurther division of the coarse clastic rocks is made on the basis of the ratio of the resistivity and natural gamma responses. This ratio appears to be sensitive to textural variations in clastic rocks, low values corresponding to fine textures and high values corresponding to coarse textures. The resistivity to natural gamma emission ratio, multiplied by an arbitrary scale factor of 100, is defined here as the "grain size index", and is used to divide the coarse grained clastic rocks into seven textural categories, as outlined in Figure 15.

The credibility of this method of inferring lithological characteristics from geophysical data is a function of three factors:

1) the nature of the theoretical foundation of the relation between the geophysical responses and the specific lithological characteristics inferred from them,

2) the degree to which the assumptions implicit in the method reflect geological realities in the area in which the method is applied and 

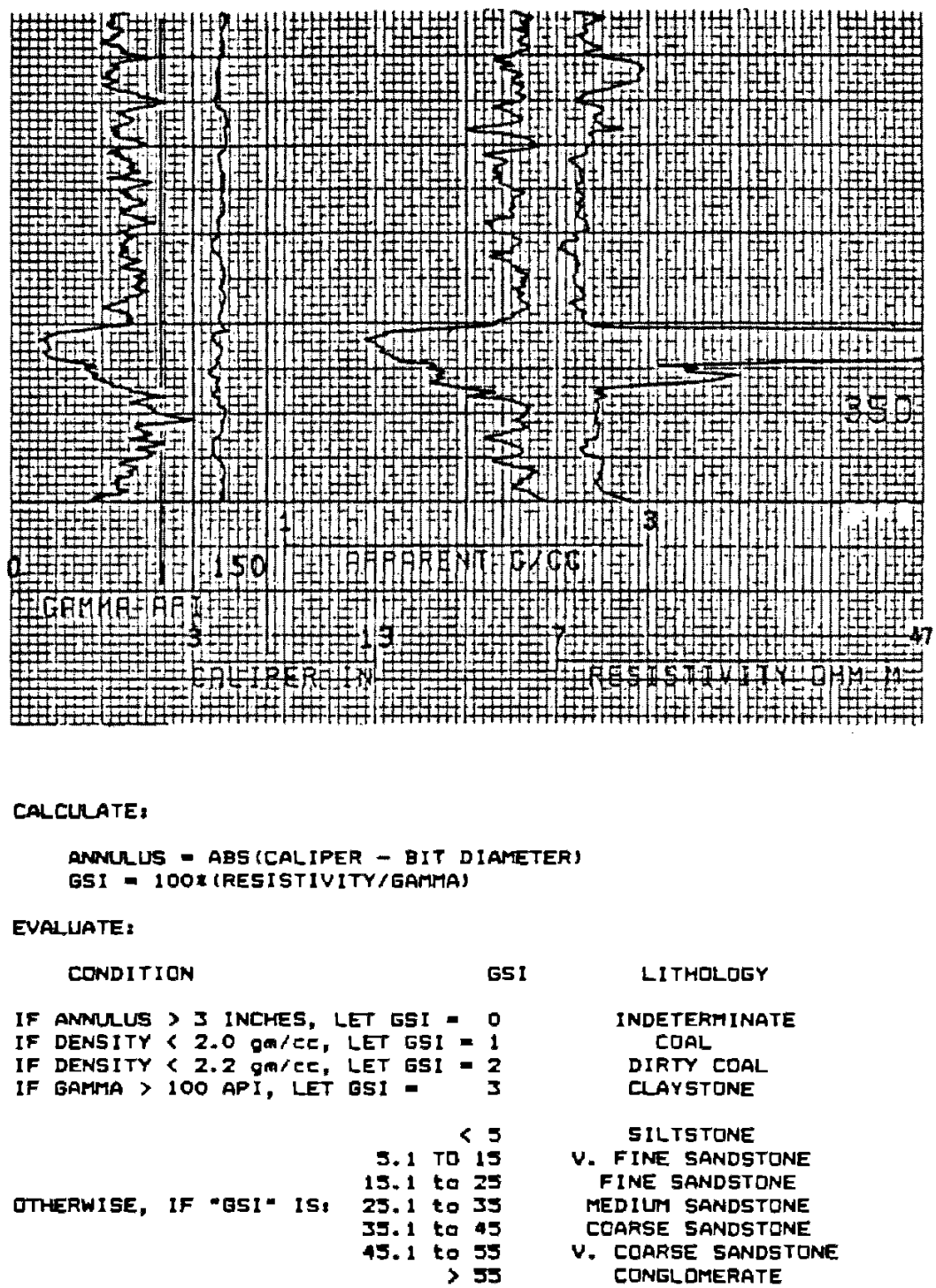

Figure 15. Classification of detrital rocks by the grain size index technique. 
3) the degree to which the method is successful in predicting lithological characteristics.

The method is based, in part, on established theoretical work, but also rests on assumptions regarding the composition of the materials and the ideal characteristics of the lithologic classes established by the method. The attenuation of induced gamma radiation is a direct consequence of the mass per unit volume of the material surrounding the source of gamma rays, and the induced gamma attenuation $\log$ is calibrated directly in gm/cc, the units of density. The radius of interaction of the induced gamma radiation employed in gamma-gamma bore-hole logging devices of the type employed in this project is on the order of 15 centimeters ( 6 inches), the accuracy of the readings is on the order of $0.1 \mathrm{gm} / \mathrm{cc}$ and the resolution is approximately 0.3 meters ( 1 foot) (Vaninetti, 1981). The cut-off values between the coal, carbonaceous shale and clastic rock categories established for this study are largely empirical, having been determined by trial and error matching of the geophysical logs of several drill holes with core logs of the same holes. For the most part, the changes in density across a sediment-coal boundary are sharp and easily identified. Difficulties arise only in those instances where very thin seams of coal are interbedded with clastic rocks. For interbeds less than 10 centimeters ( 4 inches) thick, the vertical variations in 
the properties of the rocks are below the limit of resolution of the logging device. The resulting values of density obtained from the geophysical logs lie between the actual densities of the interbedded materials. These occurrences have been interpreted as belonging to the intermediate "carbonaceous shale" lithologic category, whereas they may, in actuality, be either homogenous carbonaceous shale or finely interbedded sequences of relatively pure coal and clastic rocks, the gamma-gamma tool being unable to distinguish between the two. The method is quite efficient in recognizing coal beds more than 10 centimeters ( 4 inches) thick. In every case where such a coal seam was observed in a core or inferred from an examination of cuttings, the seam was identified independently by examination of the corresponding density $\log$.

The separation of claystones from coarse clastic rocks using natural gamma emission levels is based on the abundance of naturally radioactive trace elements (uranium, thorium, etc) in clays and the relative lack of non-clay mineral phases in claystones. The natural gamma emission response of detrital rocks is a function of the abundance of potassium-bearing minerals, chiefly the clays, feldspars and micas. Lithologic units which contain an abundance of either or all of these mineral groups would be expected to produce elevated natural gamma emission responses, and those 
which contain one or another without significant dilution by potassium-poor phases would be expected to produce extremely high natural gamma emission responses. Modal analyses of the detrital rocks in the study area indicate a relatively constant proportion of feldspar within a given grain size class (Figure 7, p. 12), so high natural gamma emission responses are unlikely to be due to the presence of unusually arkosic rocks. Claystones, on the other hand, are quite common in the stratigraphic sequence in the study area and are assumed to be the source of natural gamma emission levels in excess of 100 API units. The 100 API unit cut-off value was determined empirically, by comparing the natural gamma emission responses of a number of coreholes with the lithological descriptions contained in the core logs. The method works quite well, but is subject to interference from lithological units rich in volcanic glass. These tuffaceous units have a natural gamma response which is comparable to that of claystones and, where present, are misidentified. The division of the coarse clastic rocks into a series of textural classes based on an ordinal scale is a unique feature of the interpretative method developed for this study. Although the classes defined by the method are textural, the theoretical basis for the division is essentially compositional. The translation of compositional characteristics into textural characteristics is possible because the two are not independent variables. The 
relationship between the mineralogical composition and texture of clastic sediments is well-known (Figure 16). Within the sediments deposited by a given process from material originating from a given source, quartz is more prevalent than the predominant potassium-rich phases in coarse size classes and the reverse is true in fine size classes. Since the natural gamma emission response of a sedimentary rock is a function of its concentration of potassium-rich minerals, an inverse relationship ought to exist between grain size and natural gamma emission. That this is the case is demonstrated by the correlation between grain size and natural gamma emission responses described by Vaninetti (1981). That the natural gamma emission response is also related to other, independent factors, however, is demonstrated by the disappointing results obtained by using natural gamma emission response as the sole indicator of texture.

The resistivity of clastic rocks is dependent, to a first approximation, on the porosity of the rock, the concentration of ions in interstitial fluids and the abundance and mode of occurrence of clay minerals (Wyllie, 1963). Since the clays are excellent electrolytic conductors, their presence in a clastic rock reduces its resistivity. The clay content and the resistivity are thus inversely related, the constant of proportionality varying with the mode of occurrence of the clay. If the clay is 


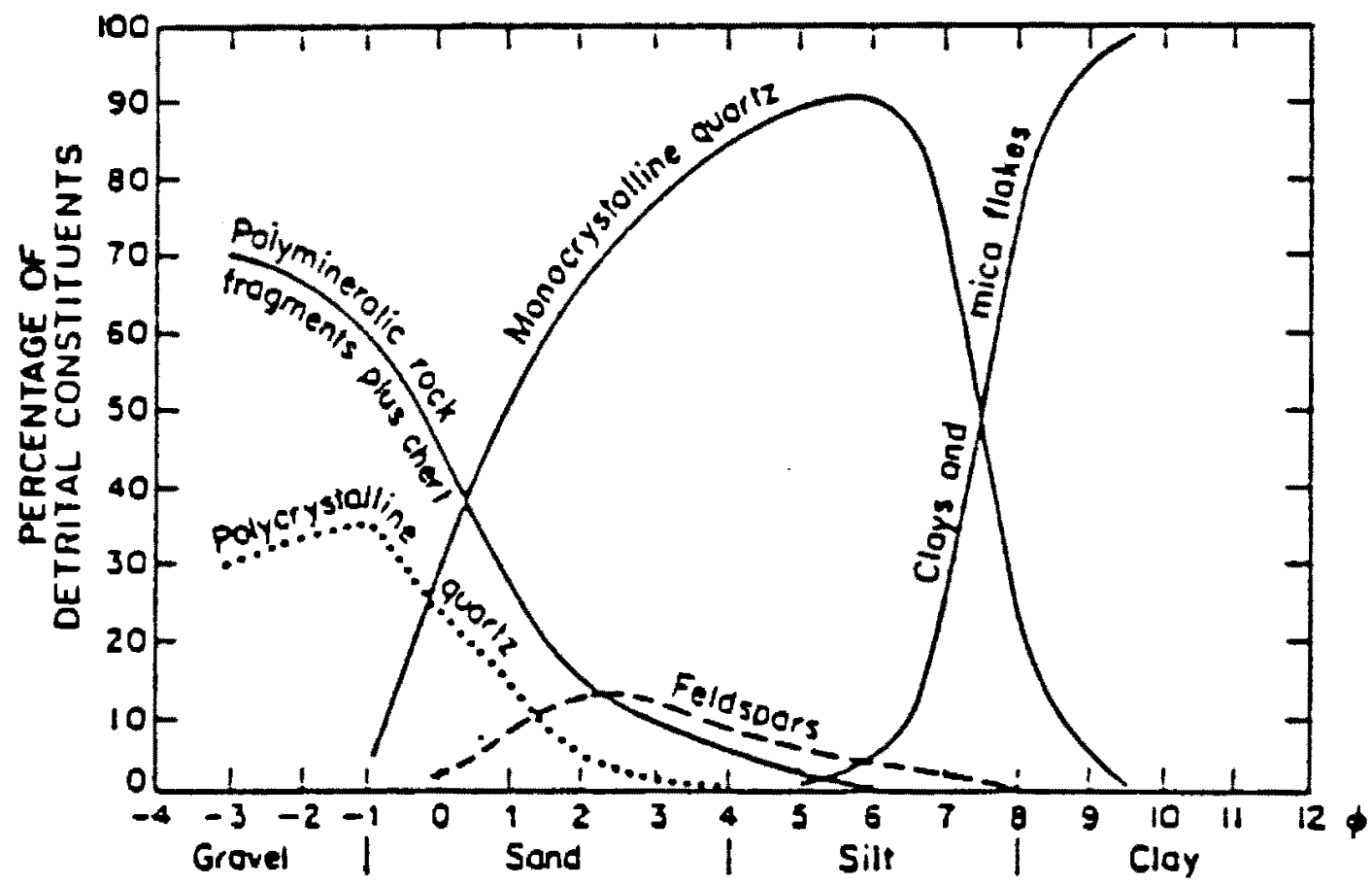

Figure 16. Relationship between modal composition and texture. 
present in isolated interstitial spaces, the proportionality constant is small and the effect is negligable. However, if continuous clay films are present, forming a conducting network which pervades the rock, then the constant of proportionality is large and the resistivity of the rock is dramatically reduced. Although the abundance of clay in a clastic rock is not normally considered to be a strict function of framework grain size, there is a relationship between framework grain size and some combination of porosity, clay content and the continuity of clay films. This can be demonstrated by the positive correlation between resistivity and framework grain size in clastic rocks saturated by the same interstitial fluid (Vaninetti, 1981). That the relationship is dependent on other factors, as well, is apparent from the disappointing results of a direct interpretation of clastic rock texture from resistivity measurements discussed earlier.

Although neither the natural gamma emission nor the resistivity response, used in isolation, are effective indicators of texture, the ratio of the two parameters seems to work quite well. Each parameter is partly responsive to texture and the combination of them into a single parameter enhances the effect of textural variation. Several important assumptions are, however, implicit in the use of the resistivity/natural gamma ratio as an indicator of texture. They are, in order of probable importance, 
1) that the quartz/feldspar ratio and the potassium feldspar/plagioclase ratio of the clastic rocks is constant throughout the section being examined,

2) that the mode of occurrence of clay within the rocks does not change appreciably from one portion of the section to another and

3) that the ionic concentration of pore water is similar throughout the section.

These assumptions are reasonable ones in the area and stratigraphic section involved in this study. Modal analysis of the rocks in the study area revealed no significant changes in the quartz-feldspar ratio with stratigraphic position. The possibility of appreciable differences in the mode of occurrence of clays between different strata is more difficult to evaluate, but the variations present appear to be related to texture within individual beds rather than to stratigraphic position. The chemistry of the ground water in the study area has not been examined, but the drill holes involved were less than 600 meters (2000 feet) deep and no overpressured zones or brines were encountered during drilling. There is no compelling reason to believe that significant variations in ground water chemistry exist in the area at these depths.

The cut-off values which divide the various clastic categories were determined by a comparison of the geophysical log of a 134 meter (440 foot) section of a 
reference drill hole (MF-09) with the results of a textural analysis of cuttings removed from the same hole. Cuttings collected at 3.3 meter (10 foot) intervals were split (50 grams), washed, disaggregated and sieved to determine their median framework grain size. The mean grain size index was calculated for corresponding 3.3 meter (10 foot) intervals in the same section and the resulting bivariate data set (Figure 17) was examined by least squares regression techniques. The resulting relationship (Figure 18) was used to determine the grain size index values corresponding to the standard particle size scale. The best fit regression line is of the second order, with a correlation coefficient of -0.92 and a goodness of fit of 0.84 . The improvement obtained by an increase in the order of the regression is not statistically significant. A runs test for autocorrelation of residual deviations from the regression line and an ANOVA examination for a depth-related trend in the residual deviation from the regression line were negative at the 99\% confidence level. This indicates that the initial assumptions underlying a linear model of the relationship between median framework grain size and the grain size index are reasonable. This is an important consideration, as the range of the available data is limited to the fine and very fine sandstones, cut-off values separating other categories being projected beyond the data base on the assumption that the linear relationship holds in 
GRAIN SIZE INDEX VERSUS DEPTH

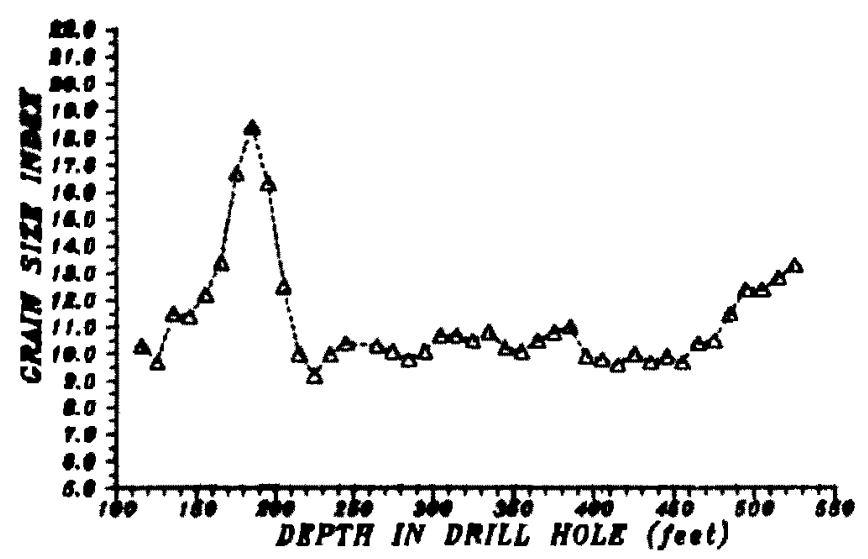

MEDIAN GRAIN SIZE VERSUS DEPTH

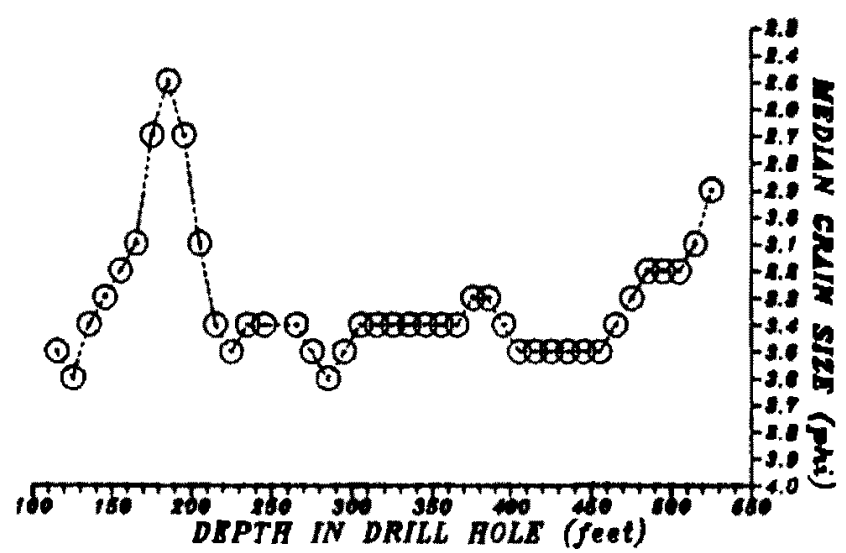

\section{GRAIN SIZE INDEX VS MEDIAN GRAIN SIZE}

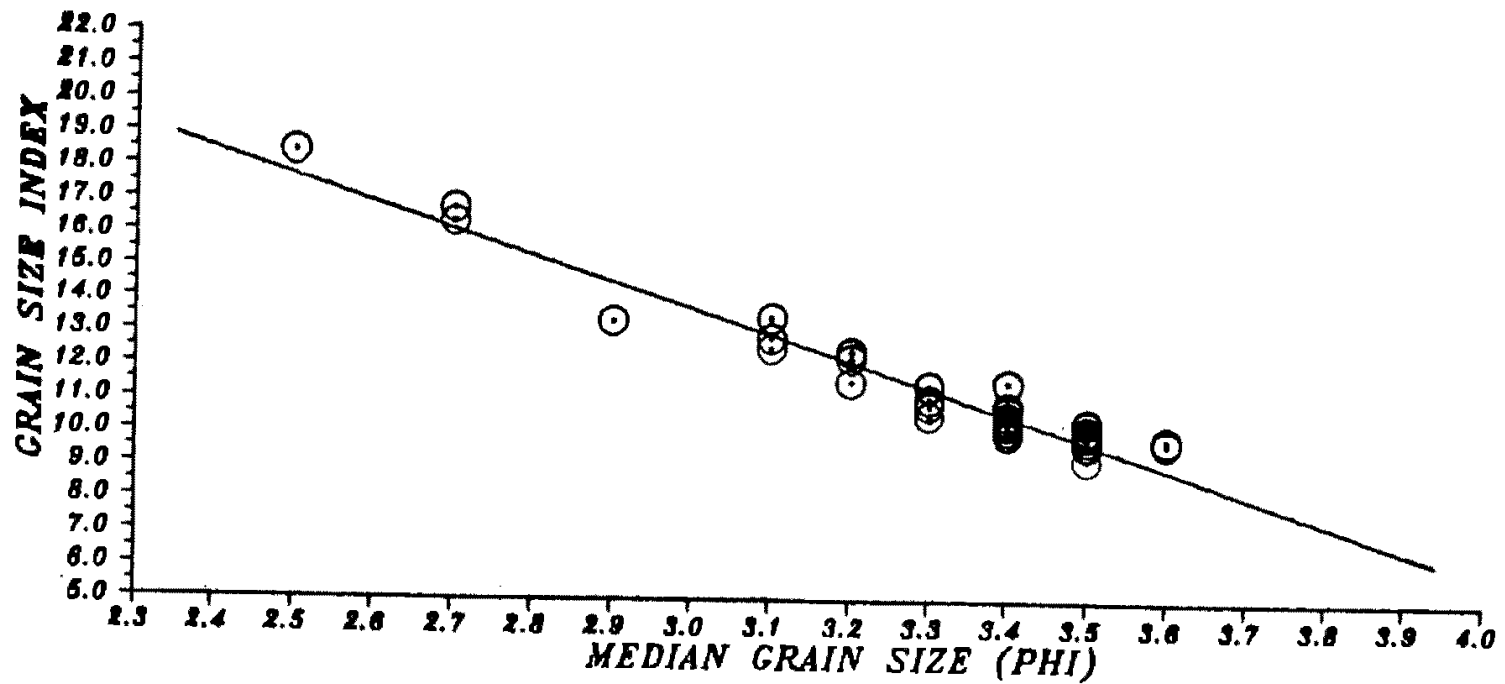

Figure 17. Comparison between grain size index and median grain size (MF-09). 


\section{CALIBRATION OF THE TECHNIQUE}

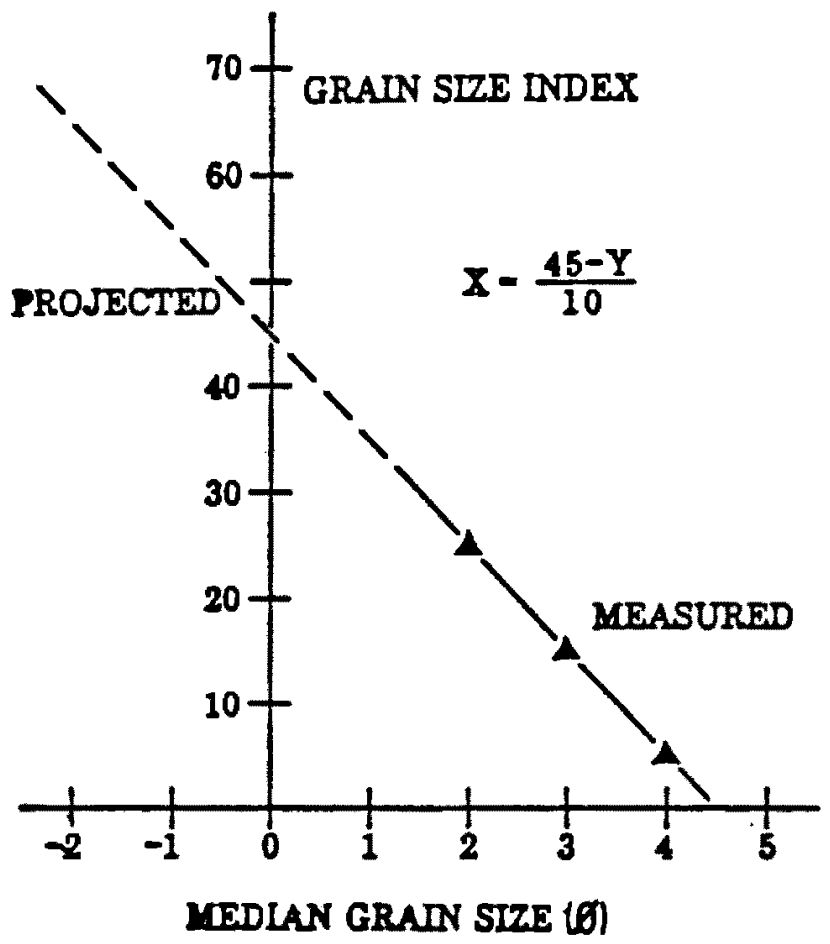

Figure 18. Calibration of grain size index to the standard phi scale. 
both directions.

The high correlation coefficient between the two independent measures of texture and the excellent fit of the regression line relating them are measures of the efficiency of the grain size index interpretation procedure. The log interpretation procedure was further tested by comparing the lithological identifications produced by the procedure with independent lithological descriptions of a core removed from drill hole MF-12C. A statistical analysis of the results was performed to test the null hypothesis of a random association. Cross-association (Figure 19) resulted in 28 matches and 20 mismatches in the 15 meter (48 foot) sequence, an efficiency of 58 per cent. Cross-association of two random sequences with lithological distributions identical to those observed yields 9 matches and 39 mismatches. A chi square computation leads to rejection of the null hypothesis at the 99 per cent confidence level. Examination of the nature and distribution of the mismatches indicates that the accuracy of the technique is approximately $+/-0.30$ meter $(1$ foot) and $+/-$ one lithological category. When the matches and mismatches are recalculated using these caveats, the efficiency of the technique is increased to approximately 90 per cent. 
A TEST OF THE TECHNLUL
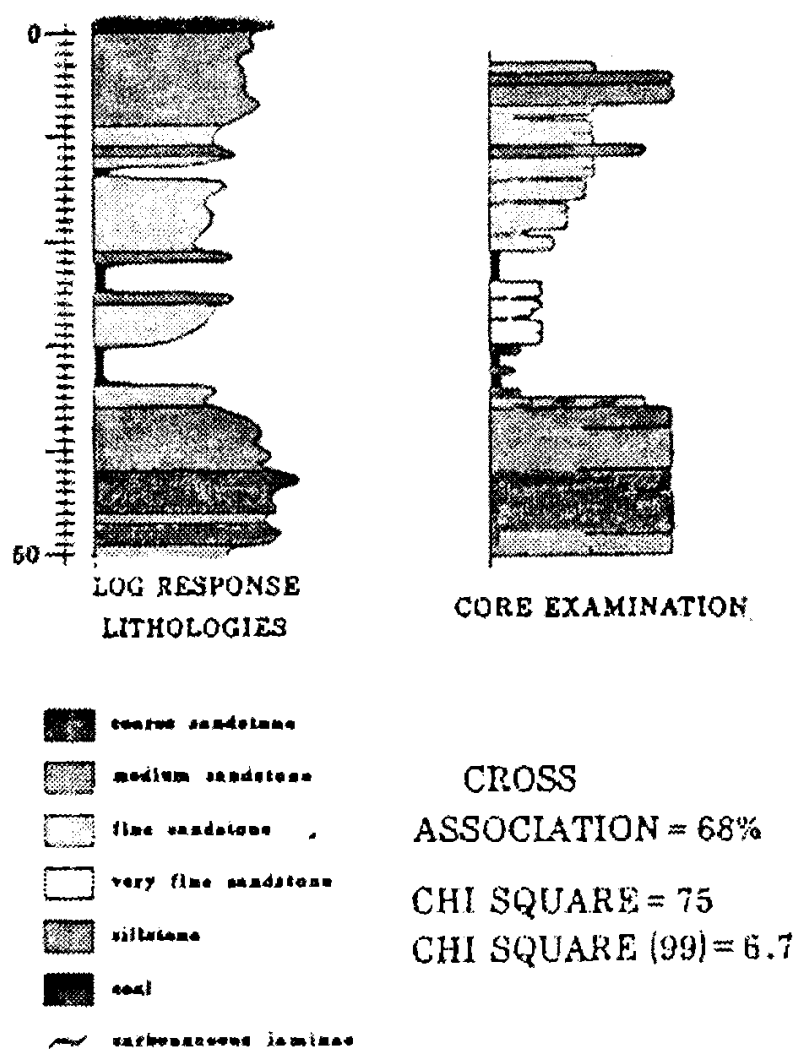

Figure 19. Comparison between calculated and observed lithologies (core hole MF-12C). 


\section{DATA REDUCTION}

Use of the lithological information generated by the interpretation of 3,300 meters $(10,000$ feet) of geophysical logs requires condensation of the information into a manageable format. The first of several condensation procedures used here involves the preparation of measured sections from the raw sequences of lithological determinations. This was carried out by calculating the elevation of the top, bottom and thickness of each of continuous lithological unit. These calculations and the data file production were handled by a micro-computer routine "LITHLOG", produced for this purpose by the author. An example of one of the "measured sections" produced by this routine is shown in Figure 20 .

From these measured sections, the data was further consolidated by grouping sequences of similar lithologies into generalized lithofacies categories. The Iithofacies categories were designed to reflect the energy of the environment of deposition. This procedure requires, however, that the categories be extended to incorporate the coal and claystone lithologies, which have been identified by other criteria. Coal and claystone have been assigned arbitrary indices of 1 and 3 respectively, on the assumption that coal accumulates under conditions of extremely low current energy and that clay is deposited under slightly 
MEASLIRED SECTION

DRILL HOLE MF-1B

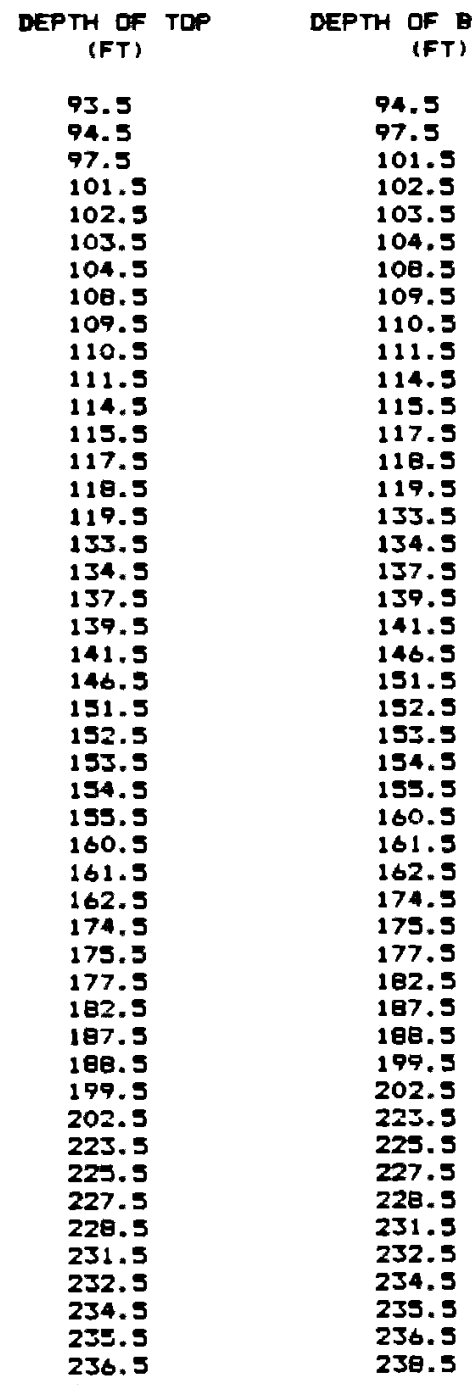

LITHOLDEY

THICKNESS

(FT)

COARSE SANDSTONE

MEDIUM SANDSTONE

FINE SANDSTONE

MEDILA SANDSTONE

FINE SANDSTONE

COAL

FINE SANDSTONE

MEDIUM SANDSTONE

FINE SANDSTONE

MEDILA SANDSTDNE

FINE SANDSTONE

FEDIUM SANDSTONE

FINE SANDSTONE

COARSE SANDSTONE

MEDIUL SANDSTONE

FINE SANDSTONE

MEDILAT SANDSTONE

F INE SANDSTONE

MEDIUM SANDSTONE COARSE SANDSTONE

FINE SANDSTONE

MEDIUM SANDSTONE

COAL

FINE SANDSTONE

mEDIUA SANDSTONE

COAL

FINE SANDSTONE *

MEDILA SANDSTONE

FINE SANDSTONE

V. FINE SANDSTONE

Fine SANDSTane

COAL

V. FIME SANDSTONE

FINE SANDSTINE

MEDIUM SANDSTONE

FINE SANDSTONE

MEDIUM SANDSTONE

FINE SANDSTONE

v. FINE SANDSTONE

FINE SANDSTOME

V. FINE SANDSTONE

FINE SANDSTONE

MEDIUM SANDSTONE

FINE SANDSTONE

V. FINE SANDSTONE

REDIUM SANDSTINE

FINE SANDSTOME

1
3
4
1
1
1
4
1
1
1
3
1
2
1
1
14
1
3
2
2
5
5
1
1
1
1
5
1
1
12
1
2
5
5
1
11
3
21
2
2
1
3
1
2
1
1
2

Fiqure 20. Example portion of a measured section produced by microcomputer program "LITHLOG" (drill hole MF-01B). 
higher, but still very low energy conditions. The grain size indices for the coarse clastic rocks have been used without modification on the assumption that the energy present during deposition of the sediment is proportional to median grain size. The new index has been designated as the "environmental energy index" to distinguish it from the grain size index, for which no values representing the claystones and coals can be calculated.

The environmental energy index, smoothed by an eleven-foot running mean, has been used to define five lithofacies. The boundaries between categories were based on the results of a first-order embedded Markov analysis of the vertical transitions from one lithology to another within the measured sections. Each of the geophysical logs was processed by a microcomputer routine, "FACIES6" , which produces the sequence of lithofacies present in the drill hole.

SPATIAL DISTRIBUTION OF LITHOLOGIES

In order to identify systematic lateral and vertical relationships between depositional environments, a series of independent techniques have been applied to the data base:

1) sand and coal isoliths were drawn for each of ten consecutive and approximately equal stratigraphic intervals covering the coal-bearing portion of the section, 
2) a series of facies maps were prepared showing the lateral distribution of the predominant lithofacies in each of twenty-three consecutive and approximately equal stratigraphic intervals covering the section, and

3) a one-step embedded Markov analysis of the lithofacies was used to model the vertical and lateral relationships between lithofacies.

The results of these techniques were tested statistically for internal consistency. The lateral and vertical relationships between lithofacies revealed by these techniques were then compiled into a generalized three-dimensional model of the depositional system.

On the assumption that the maximum contrast available is between the environments in which coarse clastic material and vegetation which served as the precursor of coal accumulated, the lateral and vertical distributions of these lithologies were chosen for detailed examination. The stratigraphic section between the Beaver Hill and the $J$ coal seams (Figure 21) was divided into ten intervals of approximately equal thickness. Since the total thickness of the stratigraphic section varies significantly from one location to another across the study area, the thickness of these intervals varies slightly between drill holes. The average value is 20 meters (60 feet). Within each stratigraphic interval, the relative proportions of coal, mudstone and sandstone in each drill-hole were calculated. 


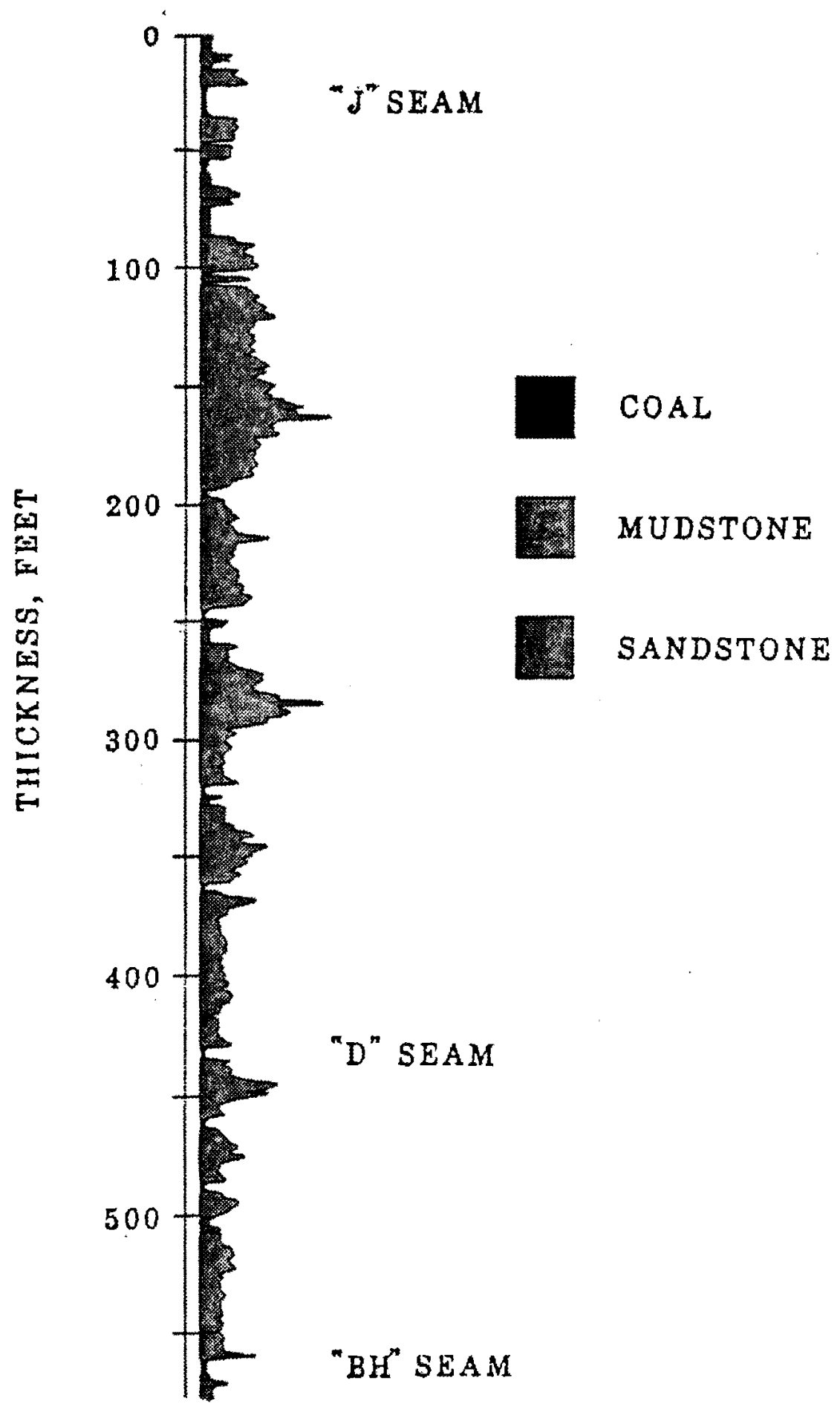

Figure 21. Lithologic character of the coal-bearing section, China Creek area. 
The results of these calculations were then contoured, producing a stratigraphically ordered series of ten coal and sand isolith maps.

An examination of the isolith maps reveals several general characteristics of the section. Within a given interval, the accumulation of sand tends to occur within broad, curvilinear belts and smaller, isolated depocenters. This tendancy is most apparent in Figure 22, in which areas containing more than 10 percent sand have been outlined. An association between the isolated depocenters and the larger, curvilinear belts is clear in several instances (intervals 2, 3, 5, 6 and 7). The distribution of coal (Figure 23) is more irregular. Curvilinear belts and isolated depocenters occur, but the occurrence of both in the same interval is rare. The spatial relationship between sand and coal within each stratigraphic interval is shown in Figure 24. In several intervals, the concentrations of coal and coarse clastic materials appear to be mutually exclusive (intervals 2 and 7$)$. In other intervals, concentrations of coal tend to occur in association with the smaller, isolated clastic depocenters (intervals 1 and 6). In general, however, the coal tends to occur along the edges of belts of sand deposition. This tendancy is best developed where narrow zones separate areas of clastic deposition (intervals 3, 4, 5, and 6).

The vertical relationships between depositional 

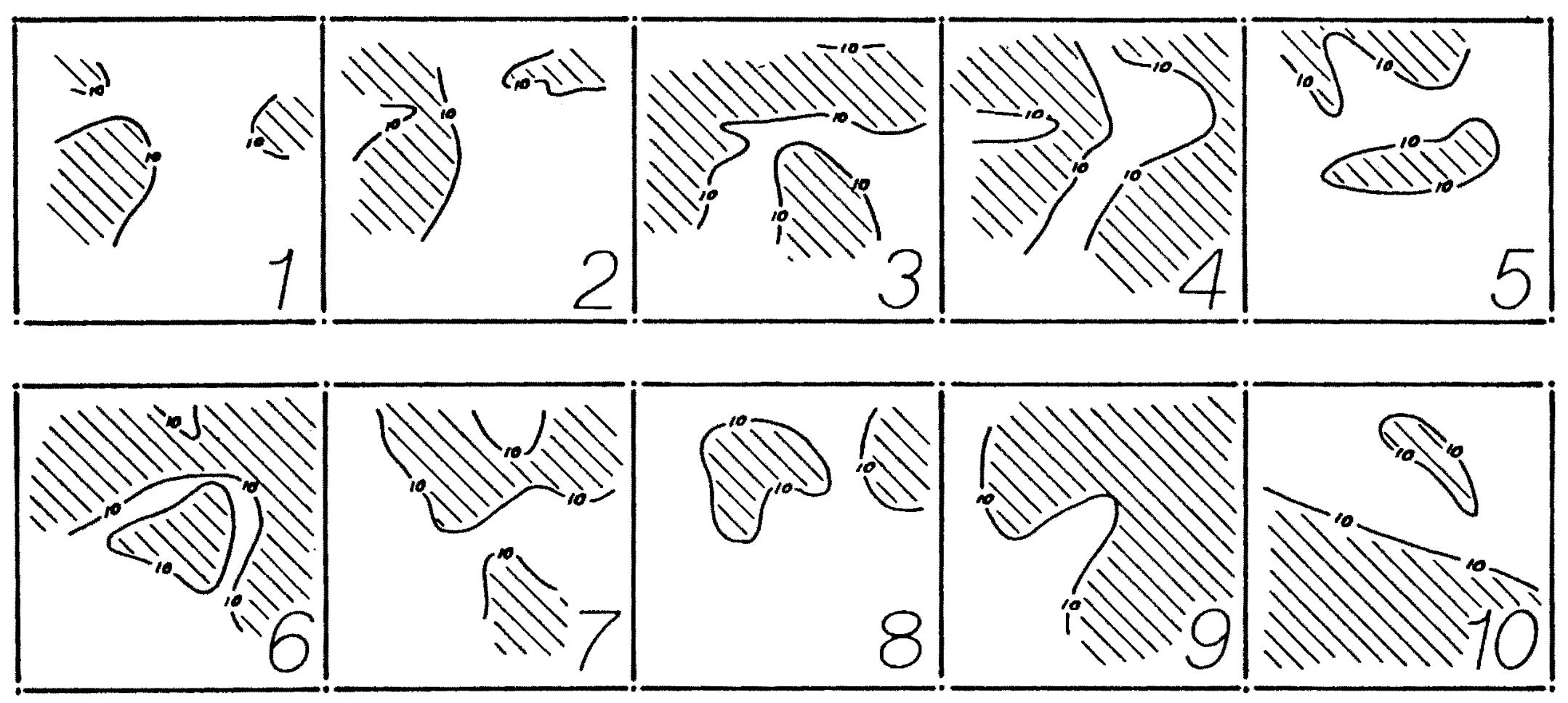

Fiqure 22. Sequential maps of coarse clastic depocenters, China Creek area. 

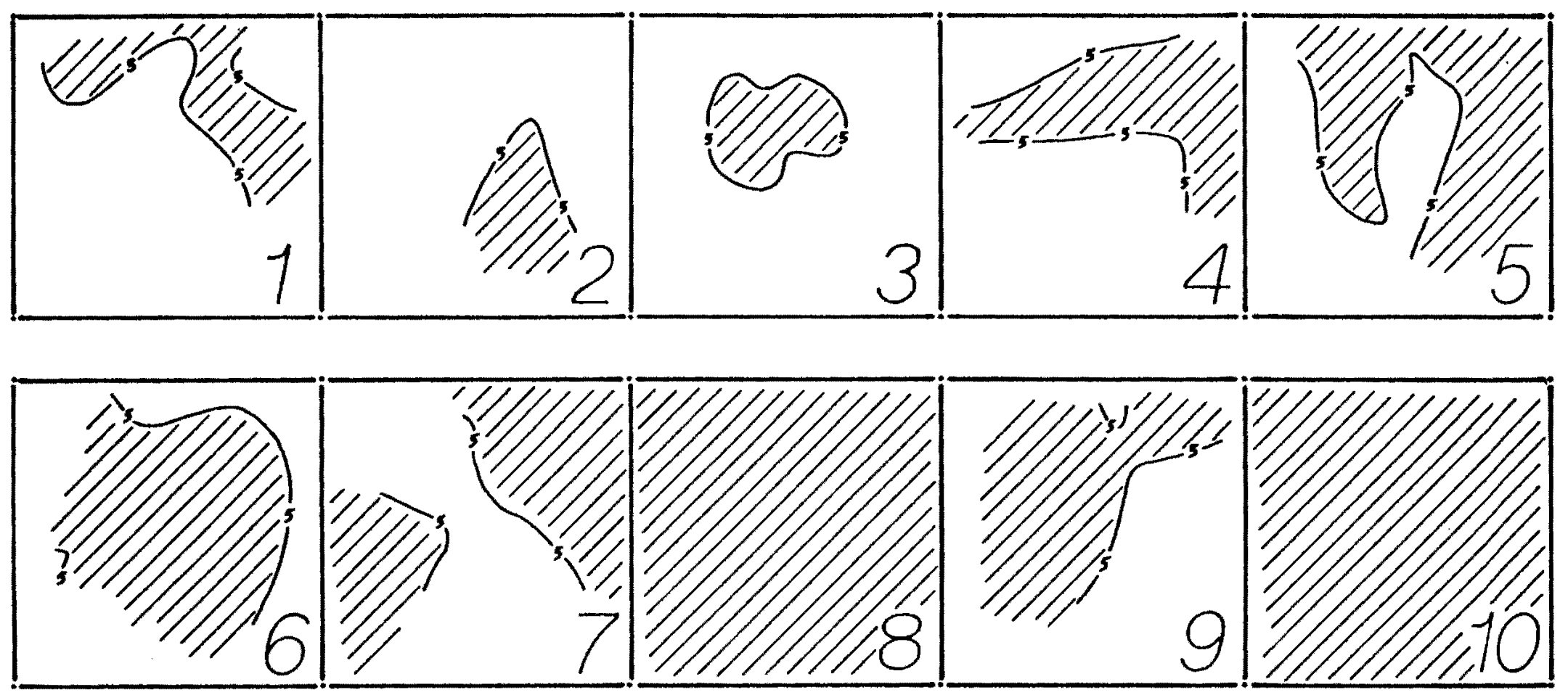

Fiqure 23. Sequential maps of coal depocenters, China Creek area. 

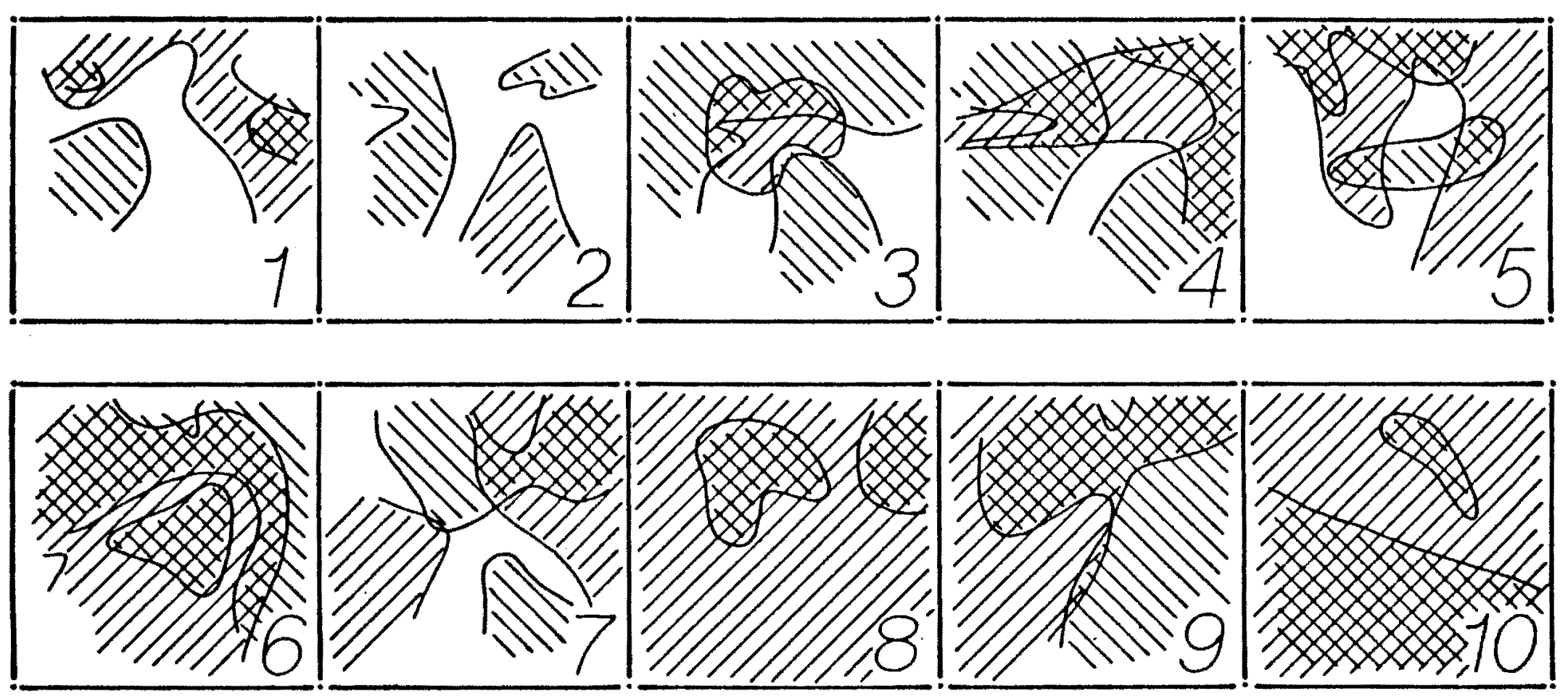

Figure 24. Superimposed sand and coal depocenters, China creek area. 
environments can be examined by comparing the depositional patterns between stratigraphic intervals. Figure 25 shows the patterns of coarse clastic deposition within each stratigraphic interval superimposed on those for the immediately preceeding stratigraphic interval. Where no dramatic change in the configuration of the patterns has occurred (the 1,2 and 7,8 sequences), the depositional system operating during that period must have been relatively stable. Where dramatic changes have occurred (the $2,3,4$, and 5 sequence), the depositional system must have undergone a considerable internal reorganization. Intermediates between these two extremes (the 5, 6, 7, and 8 sequencel represent instances where incremental, evolutionary changes were occurring in the depositional system.

\section{LITHOFACIES DEFINITION AND INTERPRETATION}

The utility of the analysis of individual lithologic states carried out above is limited by:

1) the lateral resolution of the data set,

2) the division of the section into relatively thick intervals, and

3) the choice of extreme lithological units. since additional drilling in the area is not expected, improvement in the lateral resolution of the data set is not 

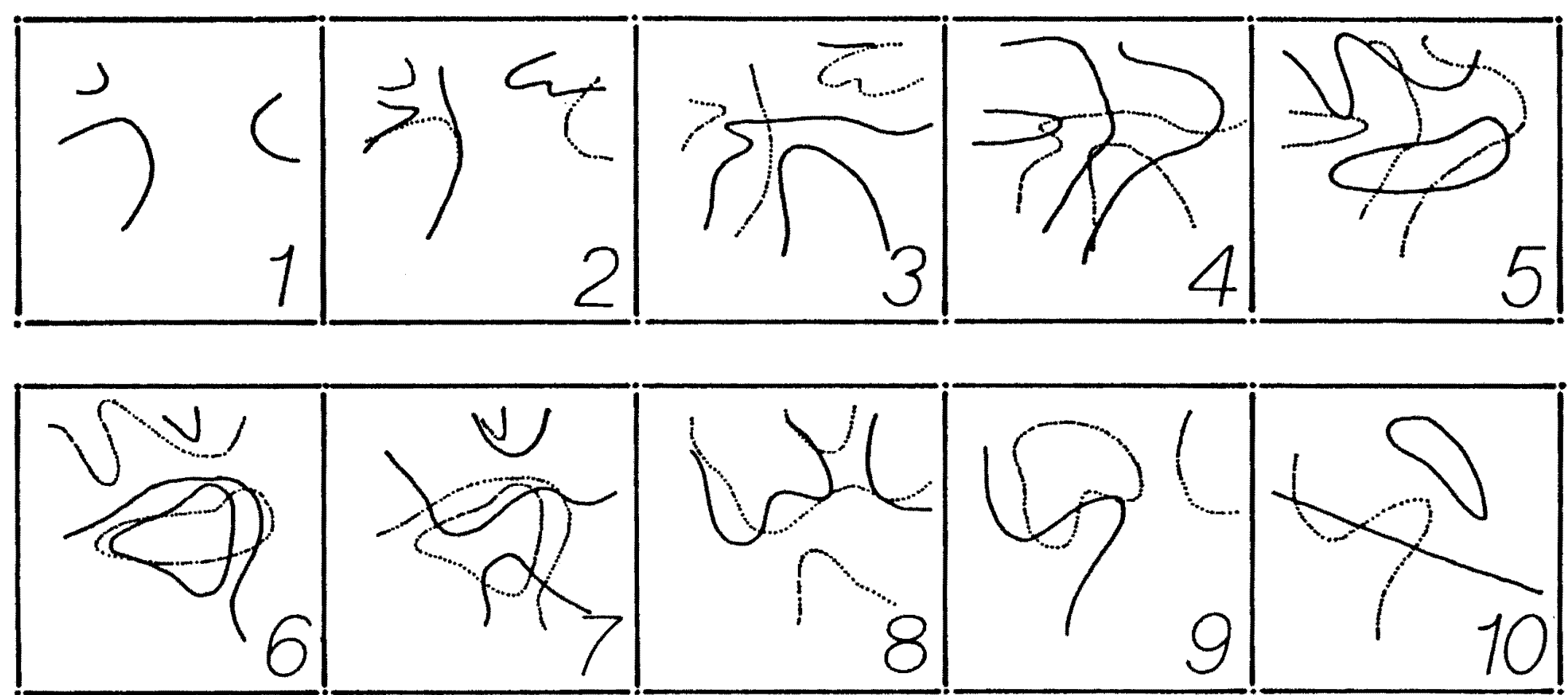

Fiqure 25. Changes in coarse clastic depocenter locations with time, China Creek area. 
possible. The vertical resolution, however, can be improved considerably by dividing the section into a larger number of thinner stratigraphic intervals. The substitution of a series of lithofacies for the generalized lithological states used above would also result is a more useful analysis.

The cut-off values used to separate lithofacies categories from one another and and a description and ranking of facies by the relative hydraulic energy of the inferred depositional environment are given in Table 2 . The depositional environments are an interpretation based on the blend of individual lithologies which comprise the units. The distribution and variability of individual lithologies within each facies in a reference drill hole are shown in Figures 26 and 27 . Although each of the facies contains several different lithologies, the modal lithology in each is distinct and both the mode and the mean environmental energy indices increase progressively from the coal swamp to the channel facies. Lithological variability within the units is an exponential function of the mean environmental energy index, with the coarser facies showing the greatest variability. The overlap between facies is, in part, an artifact caused by the smoothing of the sequence of values upon which the facies units are based, but is also a reflection of the real mixing of lithologies within the facies units. 
Table II. Summary of lithofacies characteristics, upper member, Coaledo Formation.

SUMMARY OF LITHOFACIES, CHINA CREEK AREA, UPPER MEMBER, COALEDO FORMATION

\begin{tabular}{|c|c|c|c|c|c|c|c|c|c|c|}
\hline : & & & ESSENTIRL LITH & $\mathrm{HOF}$ & ACIES & CHARACTE & $E R$ & ISTICS & & \\
\hline : & & $:$ & & : & & & & & $:$ & \\
\hline : & LITHOFACIES & : & EEI DEF INITION & $:$ & OBSER & RUED EEI & & ALUES & : & DBSERVED \\
\hline$:$ & DESIGNATION & : & (11 TERM MEAN) & : & & & & & $:$ & MODAL LITHOLOGY \\
\hline : & & : & & $:$ & & & & & : & \\
\hline : & & : & & $:$ & MEAN & : S.DEV & : & MODE & $:$ & \\
\hline : & & : & & $:-$ & $\ldots$ & + & + & - & $-:$ & \\
\hline : & & : & & $:$ & & $:$ & : & & $:$ & \\
\hline : & COAL & : & - & $:$ & 2.1 & 0.6 & : & 2 & $:$ & COAL \\
\hline : & & $:$ & & $:$ & & $:$ & $:$ & & : & \\
\hline : & & $:$ & & $:$ & & $:$ & : & & $:$ & \\
\hline$:$ & OVERBANK & : & $<=5.00$ & : & 4.6 & 2.8 & : & 3 & : & CLRY \\
\hline : & & : & & $:$ & & $:$ & : & & : & \\
\hline : & & : & & $:$ & & : & : & & : & \\
\hline$:$ & LEVEE/SPLAY & $:$ & $5.01-15.00$ & $:$ & 11.4 & 4.5 & $:$ & 13 & $:$ & VERY FINE SAND \\
\hline$:$ & & : & & $:$ & & $:$ & $:$ & & $:$ & \\
\hline$:$ & & : & & : & & $:$ & $\therefore$ & & : & \\
\hline : & BAR & : & $15.01-20.00$ & $:$ & 18.7 & 4.1 & : & 14 & $:$ & FINE SAND \\
\hline : & & $:$ & & $:$ & & $:$ & : & & $:$ & \\
\hline$:$ & & $:$ & & $:$ & & $:$ & $:$ & & $:$ & \\
\hline : & CHANNEL & : & $>=20.01$ & $:$ & 27.3 & 5.2 & : & 24 & $:$ & FINE SAND \\
\hline : & & : & & : & & : & : & & : & \\
\hline
\end{tabular}

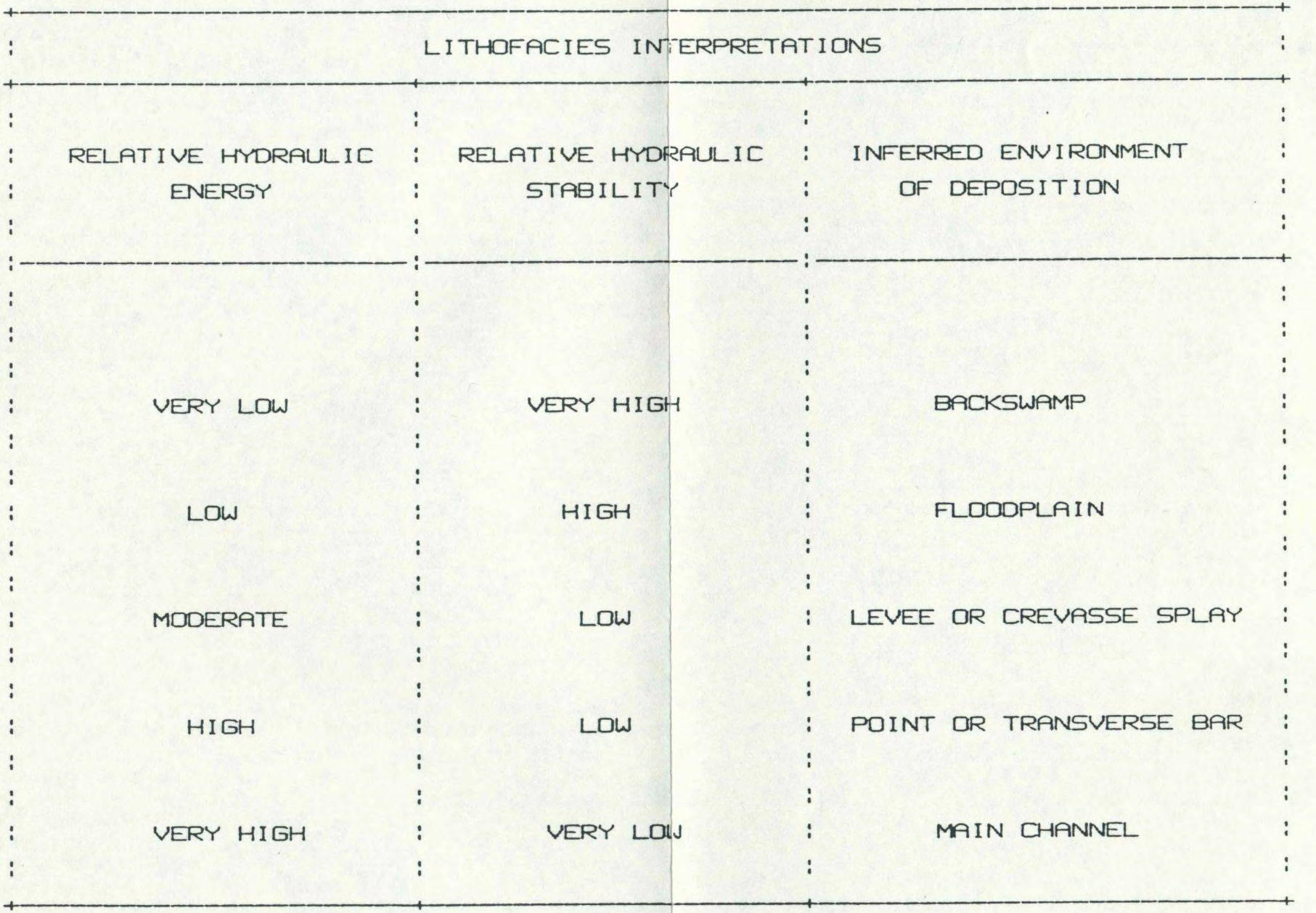




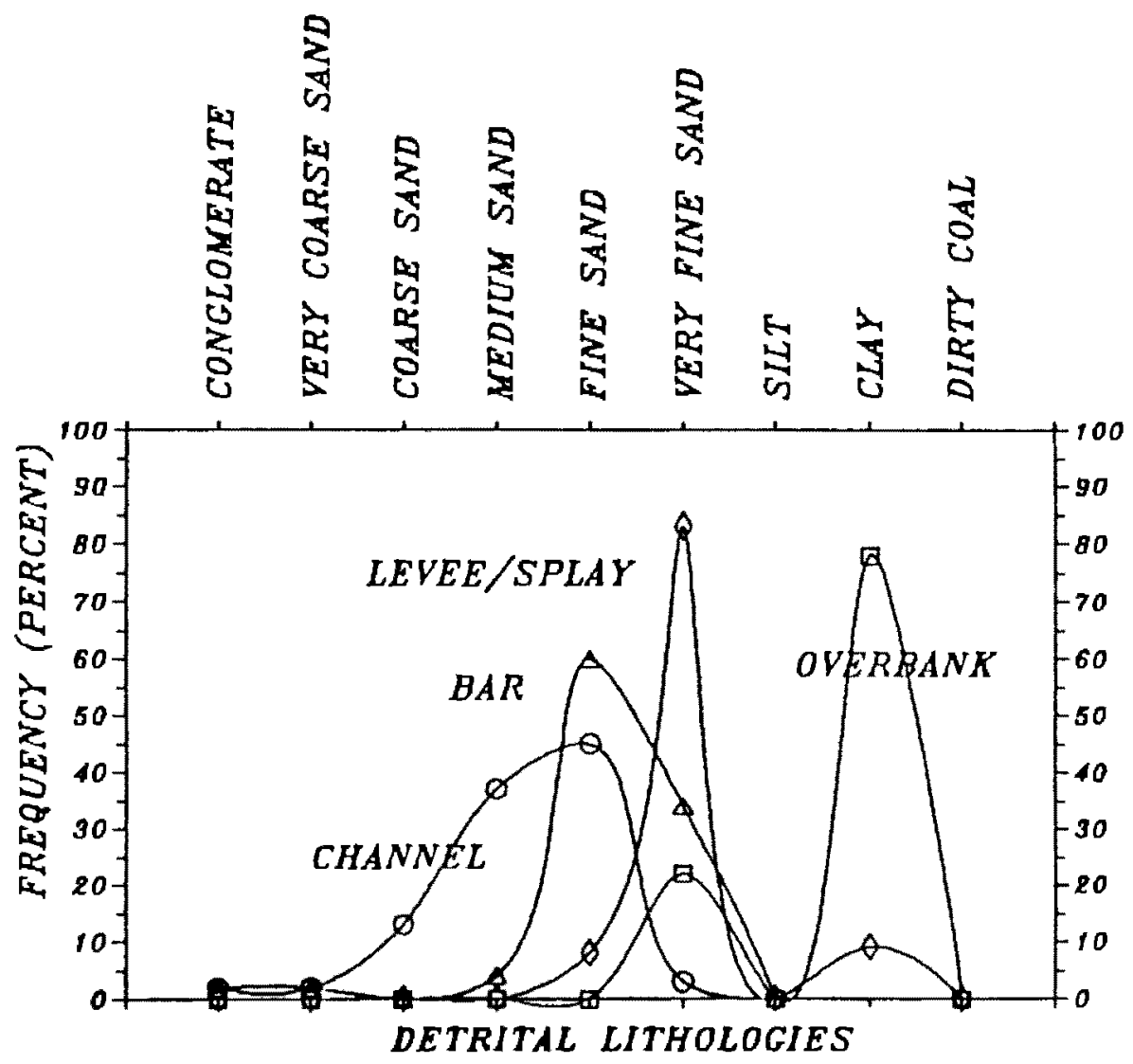

Figure 26. Distribution of lithologies in each ithofacies category. 
ENVIRONMENTAL ENERGY INDEX

MEAN VS STANDARD DEVIATION

(531 determinations, $C B-11$ )

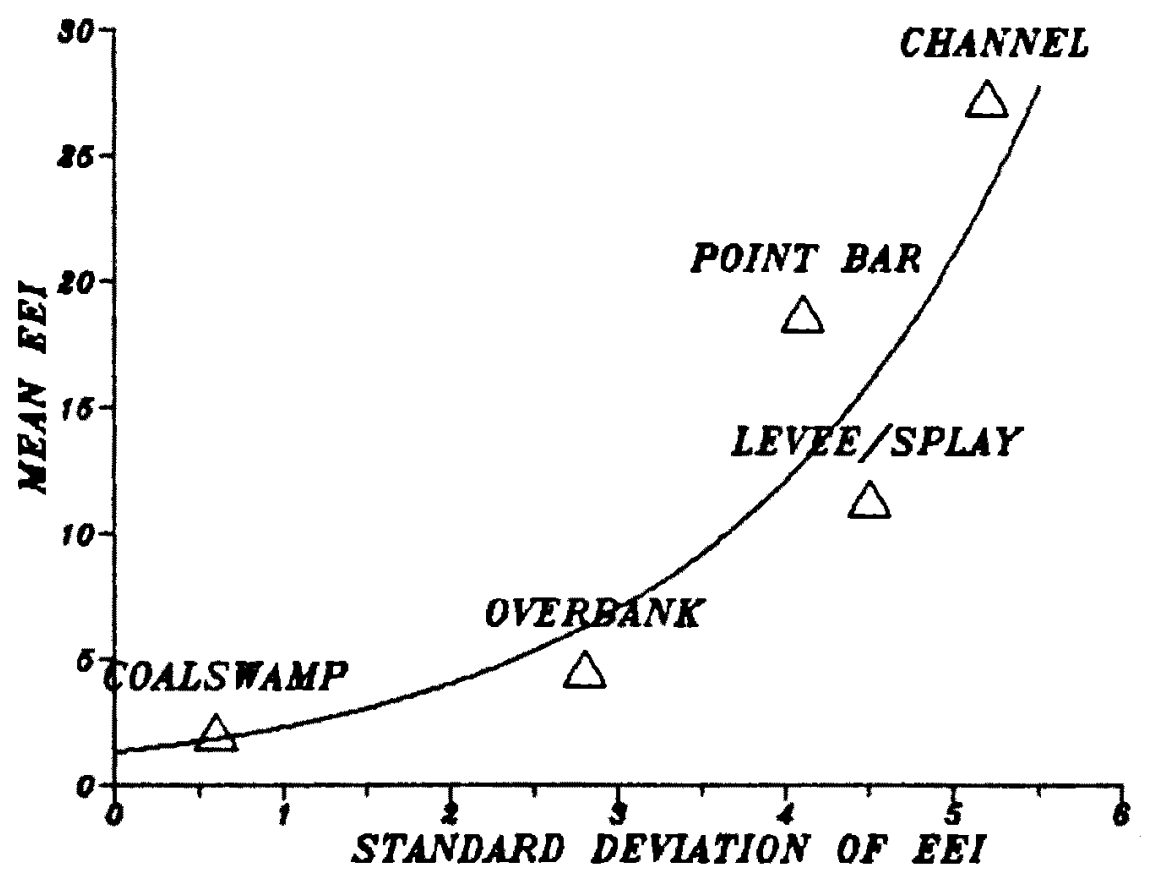

Figure 27. Mean and standard deviation of environmental energy index in individual lithofacies. 
MARROV ANALYSIS OF LITHOFACIES SEQUENCES

A one-step, embedded Markov analysis (Carr and others, 1965; Gingerich, 1969; Davis, 1986) of the transitions between the lithofacies units was carried out to determine whether or not systematic relationships among the facies units exist and to characterize them. The calculations for the Markov analysis of lithologic states were performed by a microcomputer program "MARKOV", which tallies the number of transitions between the various lithofacies and determines the probability of each transition. The resulting probability matrix is compared with the probability matrix generated by considering the transitions between lithofacies to be random. The two probability matrices are compared with one another using a variation of the chi square test suggested by Gingerich (1969). To the extent that the probability matrices are different, the lithofacies sequences are systematic. Transition probabilities which are larger than the probability for the same transition occurring at random identify "favored" transitions, those which occur less often than would be expected by random succession are "supressed" to some degree. The pattern of favored and supressed transitions produces an idealized sequence of facies transitions. This sequence is peculiar to the depositional 
system in operation during sedimentation and can be used to reconstruct it.

The initial results of the Markov analysis are listed in Table 3, a summary matrix which catalogs the difference between the actual and random succession probability matrices. Negative values in this matrix identify "supressed" transitions and positive values identify "favored" transitions. A chi square test of the null hypothesis of no significant difference between the observed probability matrix and the random succession matrix results in rejection at the 998 significance level, an indication that the overall succession of lithofacies is systematic. This implies that the values within the difference matrix as a whole are significantly different from zero, but does not identify those individual values responsible for the difference. In this analysis, all positive probability differences are considered to indicate significantly favored transitions and all negative probability differences are considered to represent significantly supressed transitions. This convention is conservative, ensuring that no significant transitions are neglected.

of the 20 possible inter-facies transitions, 9 are favored and 11 are supressed. In general, transitions between similar facies units are favored and transitions from facies with larger environmental energy indices to those with smaller environmental energy indices (fining 
Table III. Transition probability difference matrix, China Creek area.

OUERLYING STATE

屎

OUERBANK LEVEE/SPLAY POINT BAR CHANNEL COALSWAMP

OUERBANK

ZERO

$+0.17$

$-0.40$

$-0.15$

$+0.38$

LEVEE / SPLAY

$-0.05$

ZERO

$-0.29$

$-0.12$

$+0.46$

POINT BAR

$-0.22$

$+0.17$

ZERO

$+0.11$

$-0.05$

CHANNEL

$-0.14$

$-0.06$

$+0.11$

ZERO

$+0.10$

COALSWAMP

$+0.14$

$+0.23$

$-0.32$

$-0.06$

ZERO 
upwards sequences) are more common and more strongly favored than the reverse.

The transition probability difference values which correspond to individual facies unit pairs can be used to quantify the spatial relationships between pairs. Two values can be calculated for each facies pair, one corresponding to the first facies overlying the second, the other corresponding to the reverse sequence. The sum of these values measures the degree to which the two facies are inter-related. Since modelling emphasizes favored transitions, the sum of the positive transition probability differences for facies unit pairs is of special interest. This sum, which may range from 0 to 2 , is referred to here as the "fidelity" of the facies pair. Within this range, large values indicate close relationships and small values indicate random associations. Since fidelity is the sum of two transition probability differences, intermediate values may be the result of the addition of two small values or the presence of a single large value. The interpretation of the relationship of the pair is quite different in these two cases, so it is important to eliminate the ambiguity. Fidelity values which are the result of a single transition probability difference are accompanied by the symbol "*" to distinguish them from fidelity values which are the result of mutually favored transitions.

A graphical representation of favored transitions is 
shown in Figure 28. Favored upward transitions are indicated by arrows from the underlying member of the facies pair to the overlying member of the pair. Positive transition probability differences are indicated. The general tendancy for fining upward is clear, as are the mutually favored relationships between several facies pairs. Where two units have a mutually favored transition relationship, a laterally interfingering relationship is inferred. Where only one of the transitions between members of a pair is favored, a lateral replacement of the lower facies by the upper facies is inferred. In either case, a straight-forward application of Walther's Law allows the vertical transitions to be translated into horizontal transitions. This can be used to model the spatial relationships among facies. The frequency of occurrence, mean thickness and fidelity values of those facies pairs which are associated can then be used to quantify the model. Figure 29 is a summary of the facies units present in the study area, the frequency with which they occur, their mean thicknesses and the proportion of the section accounted for by each. From an examination of the frequencies of occurrence, it is apparent that some are much more common in the section than others. It is also clear that the thickness of the facies units vary considerably. 


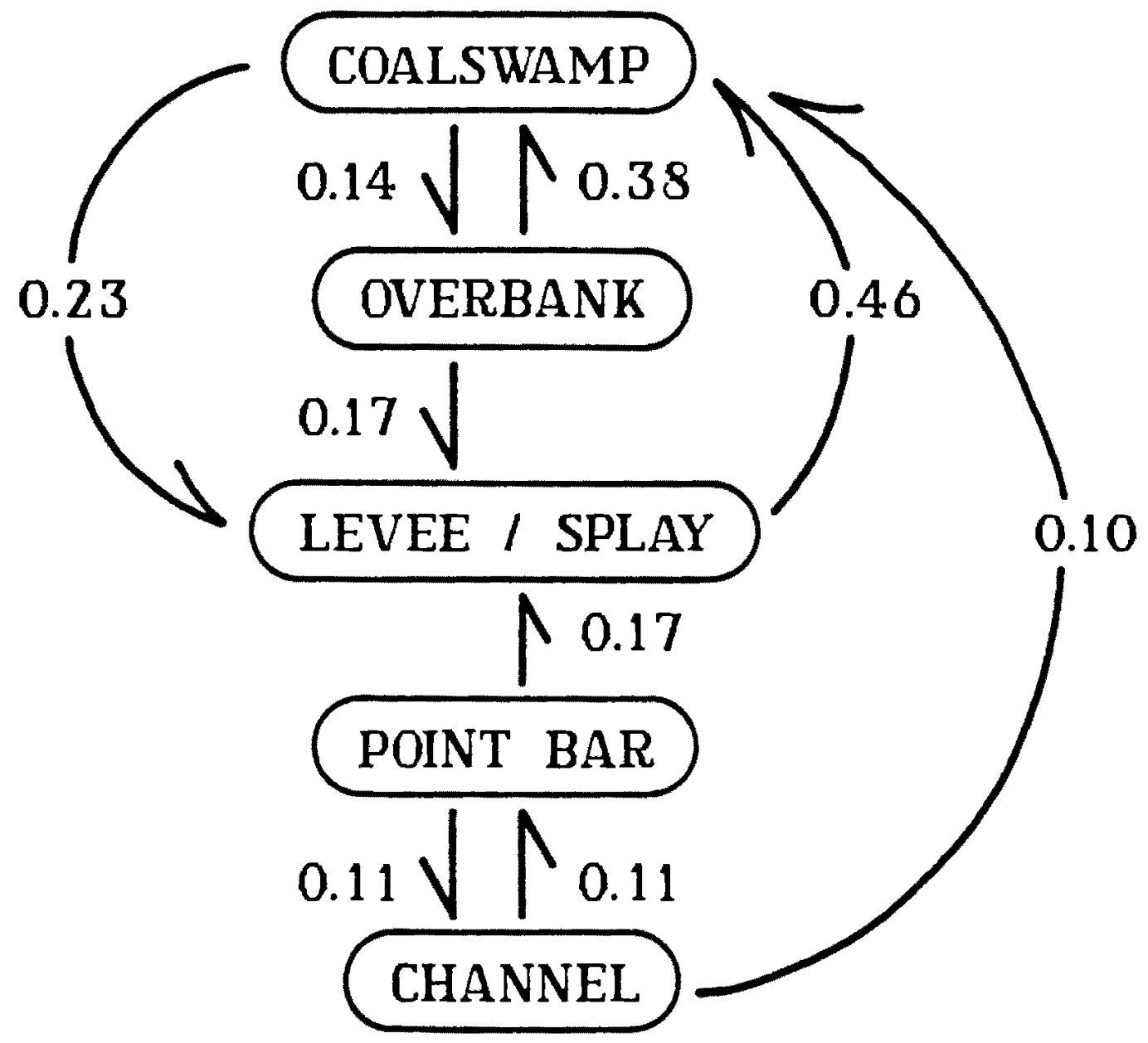

Fiqure 28. Graphical summary of favored upwards transition probabilities among lithofacies, China Creek area. 

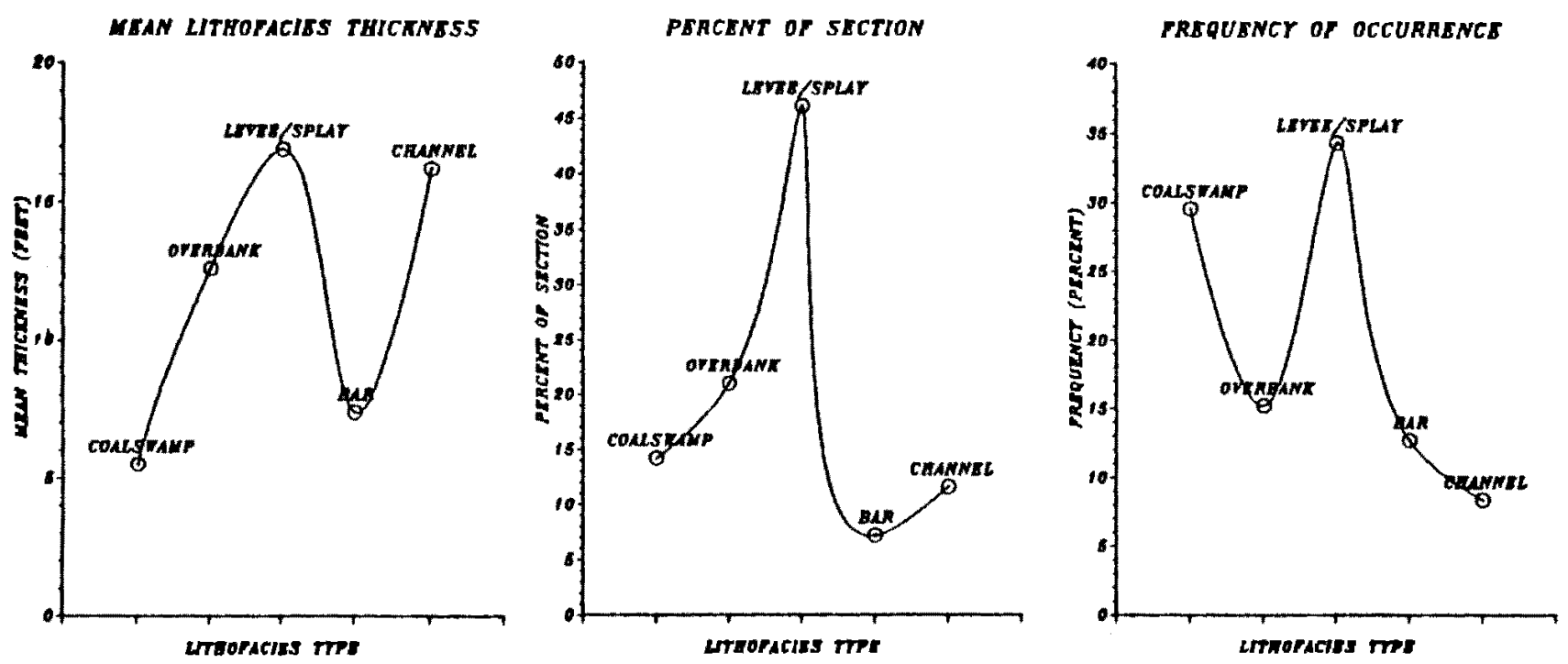

Figure 29. Mean thickness, frequency and proportions of lithofacies, China Creek 
THREE DIMENSIONAL LITHOFACIES MODEL

Based on this information, Figure 30 outlines the facies relationships which would be expected if the area under study were seen in cross-section. Two distinct types of relationships must be involked to account for the observed transition probabilities. In the first, a generally fining upward sequence in which levee deposits separate interfingering channel and point bar deposits from interfingering coal and overbank deposits account for the most common transitions. A second set of relationships, in which coal swamp deposits directly overlie channel deposits is necessary to account for the remaining observations. The idealized sequence is asymmetrical in several respects. Although channel and point bar deposits interfinger freely, as do coalswamp and overbank deposits, the same is not true of the other facies units. Levee/crevasse splay deposits systematically overlie both point bar deposits and overbank deposits, but neither of these systematically overlie levee and crevasse splay deposits. This asymmetry implies that levee deposits form a buffer between channel-point bar and the coalswamp-overbank complexes.

These models can be combined, if assumptions as to the geometry of at least one of the facies units are made. Figure 31 shows a generalized map view of the facies units 
LITHOFACIES MODEL, CHINA CREEK AREA

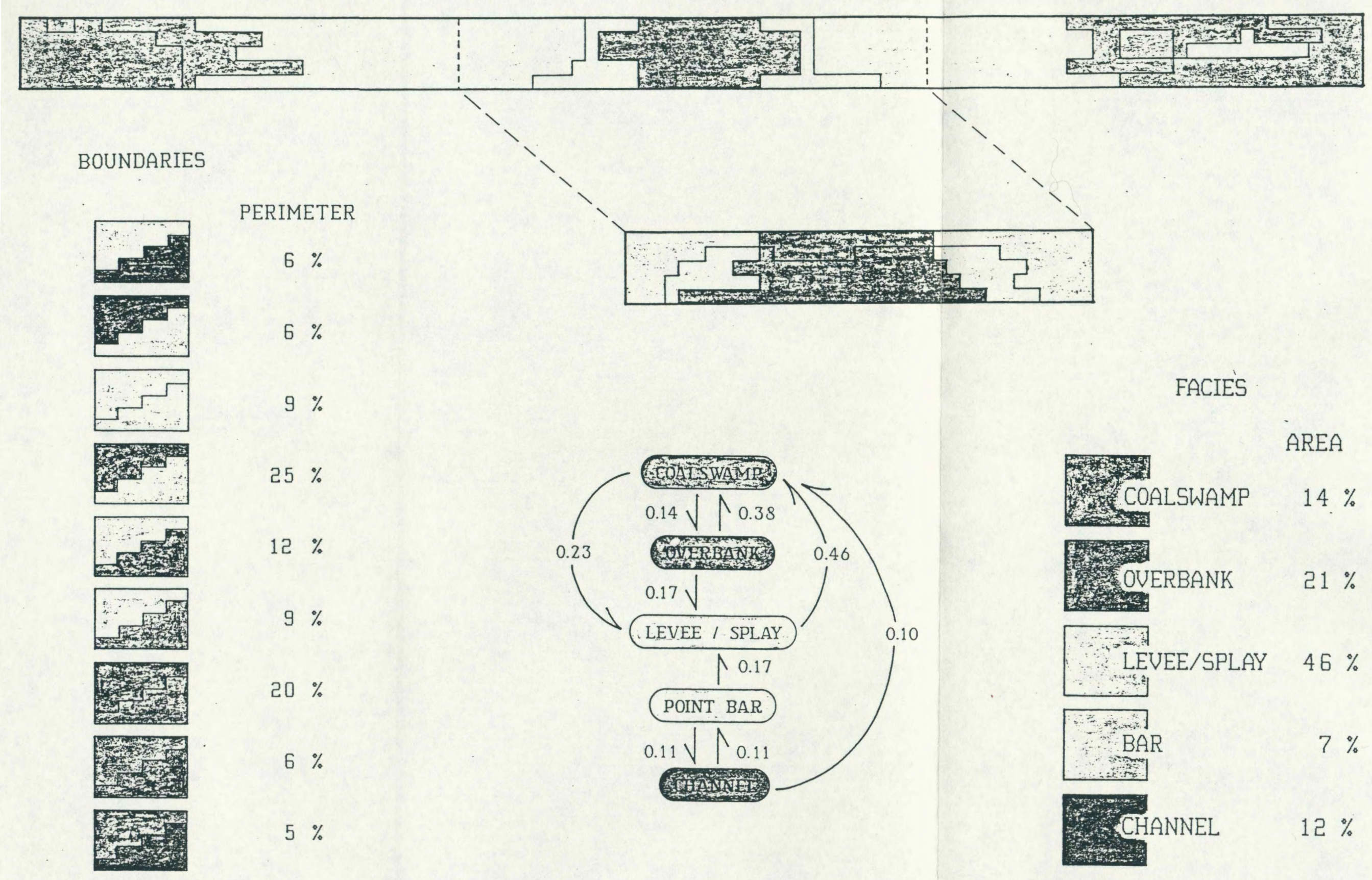

Figure 30. Modeled cross-sectional relationships among lithofacies, China Creek area. 
based on the linear, mirror-image geometry which might be expected in the immediate vicinity of a single fluvial

channel. The lateral transitions between facies are fixed by the results of the Markov analysis, only favored transitions being incorporated into the model. The length of the inter-facies contacts are directly proportional to the fidelity of the facies involved and the area occupied by each facies unit is proportional to the frequency with which it occurs within the section.

Since the model represents a single depositional episode, which is repeated many times to produce the observed stratigraphic package, the thicknesses of each of the facies within the model should be comparable to the observed mean thicknesses of those same facies within the study area. The mean thickness of each of facies in the cross-sectional model is obtained by averaging the thickness of each facies in a series of transects through the cross-sectional model. The actual mean thickness of each facies in the study area is obtained by averaging the thickness of the facies observed in drill holes. A comparison of the two (Figure 32 ) is a test of the model. The relationship between the two sets of thickness is linear, with a correlation coefficient of 0.94 . A t-test of the null hypothesis that the correlation coefficient is equal to zero results in rejection at the 99.5 * confidence level. 


\section{MODELED VS ACTUAL THICKNESSES}

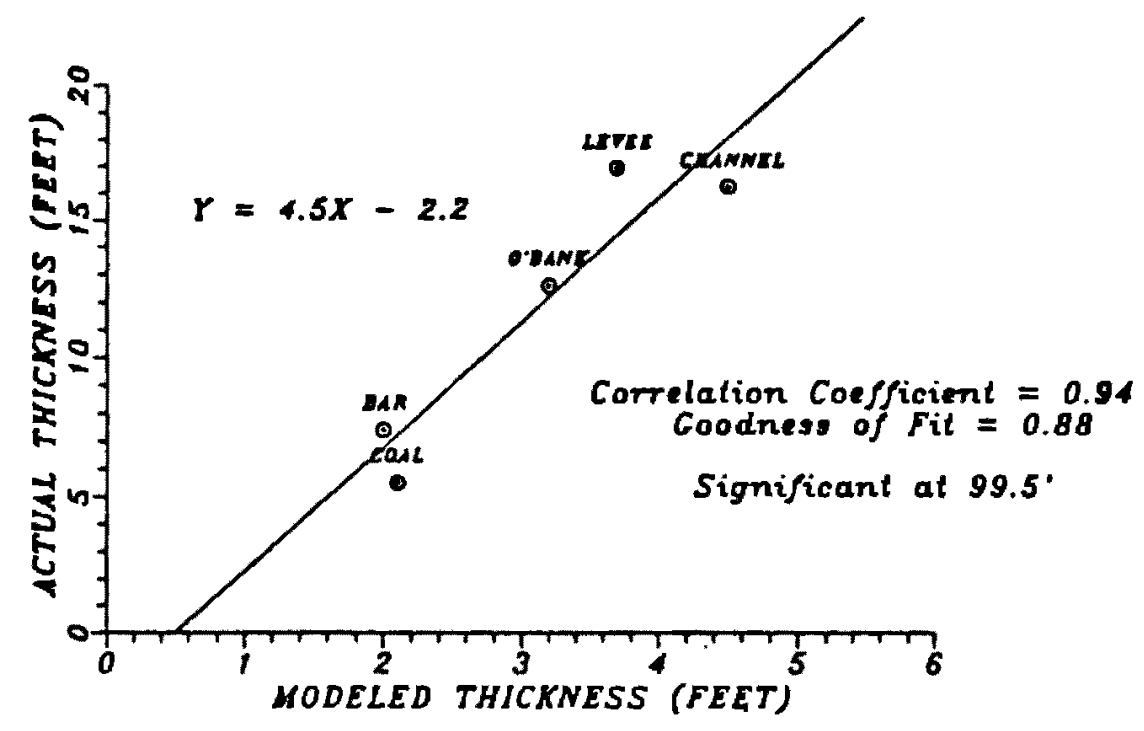

Figure 32. Comparison between modeled and actual lithofacies thicknesses. 


\section{SPATIAL DISTRIBUTION OF LITHOFACIES}

The actual distribution of lithofacies in time and space can be examined by constructing lithofacies maps in a manner similar to that used in the construction of the isolith maps. The section was divided into a set of 22 consecutive, stratigraphic intervals of approximately equal thickness. The distribution of lithofacies within each interval was then drawn in map view. The result is an ordered series of maps outlining the distribution of lithofacies as some function of time. Thin, 8 meter (25 foot) intervals were used to minimize the averaging effect of facies changes and to ensure that the material in each drill hole would fall into a single lithofacies category. The quantum nature of the data base requires that lateral transitions between adjacent, coeval lithofacies be indicated as sharp boundaries along lines between the drill holes. This requires that an artificial set of inter-facies boundaries be established along the lines which divide the area into polygons centered on the drill hole locations. The sharp changes between facies along these polygon boundaries give the maps a marked "cartoon" appearance.

The thickness of the intervals chosen for the analysis and the limit of lateral resolution resulting from the distribution of drill holes require modification of the lithofacies units. There are significant inequalities 
in thickness, frequency, and volume among the lithofacies. The coal swamp, overbank, bar, and channel lithofacies are thin or occur infrequently, or both. Individually, they constitute a relatively small percent of the total section. It is unlikely, therefore, that the areal extent of any of them in any interval would be determinable with reasonable accurracy, given the distribution of drill holes. Fortunately, the hydraulic characteristics of the environments reflected by these lithofacies are such that little information would be lost if they were consolidated into two composites, a lithofacies composed of the coal swamp and overbank lithofacies and a lithofacies composed of the bar and channel lithofacies. These composites constitute 35 and 19 percent, respectively, of the section, the remaining 46 percent falling into the levee/splay lithofacies. These proportions are more likely to be visible at a lateral resolution of 0.8 kilometers 10.5 miles). A circle with a radius of 0.8 kilometers 10.5 miles) constitutes 9 percent of the study area. The series of lithofacies maps are reproduced in Figure 33. Although some of the patterns are complex, a number of broad generalizations can be can be made. The most useful of these are:

1) In most of the intervals, the coalswamp/overbank lithofacies occupies most of the area and serves as a 

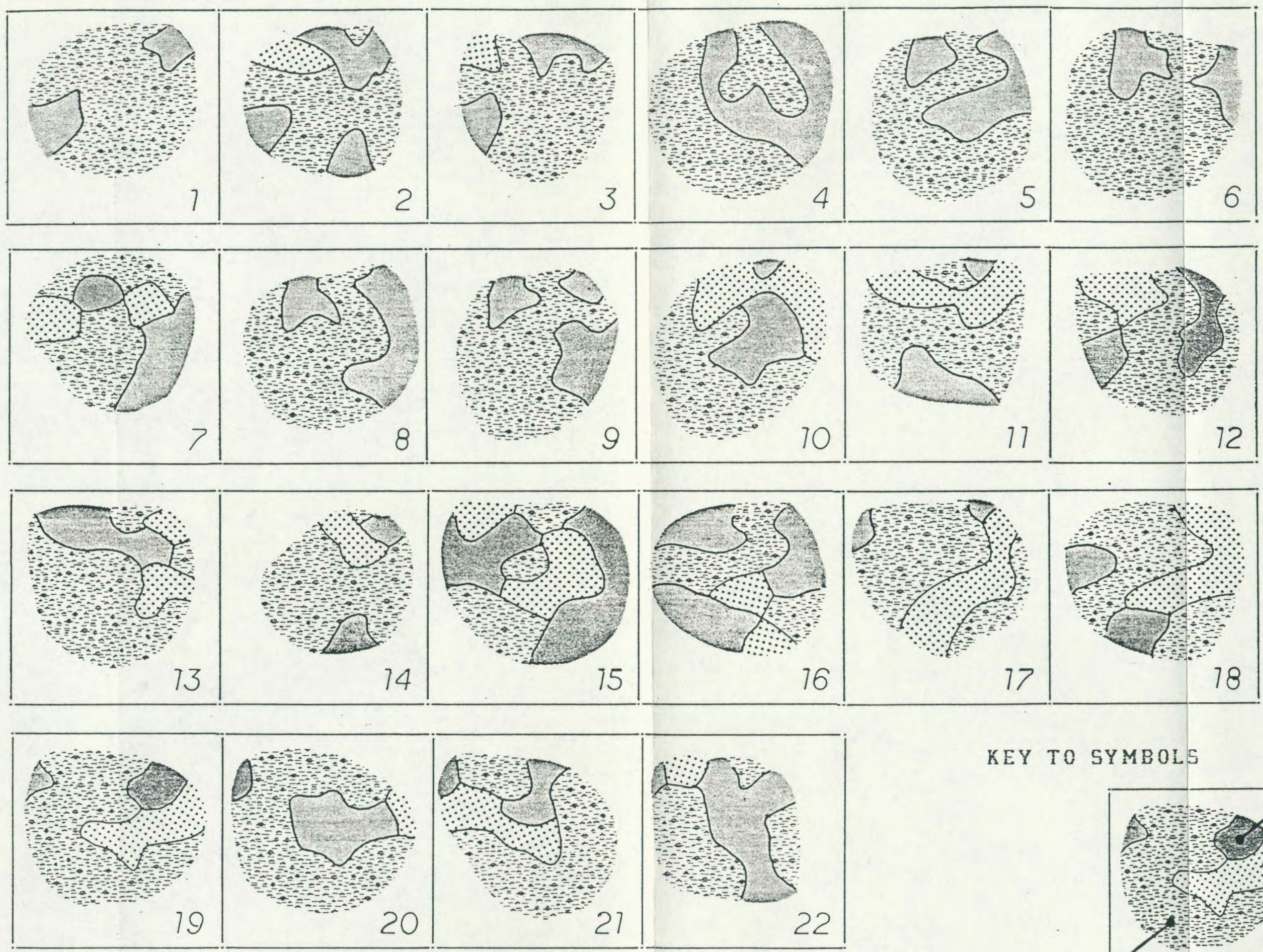

KEY TO SYMBOLS

COALSWAMP/OUERBANK

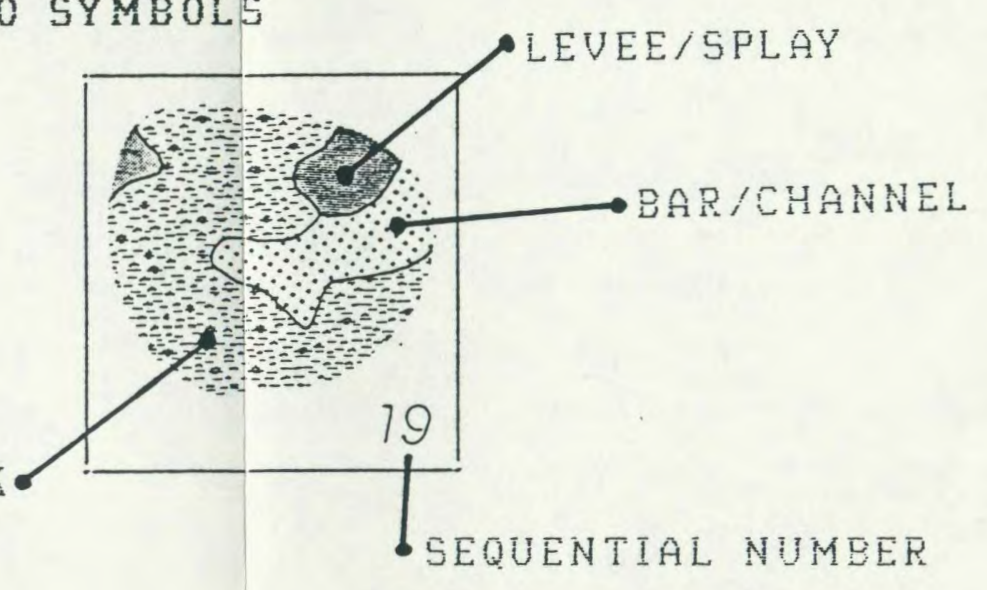

Figure 33. Sequential maps of the actual distribution of lithofacies, 
background in which the other lithofacies occur as curvilinear bands or isolated depocenters.

2) In those intervals where both bar/channel and levee/splay lithofacies occur, they appear to be related spatially. They commonly share a linear boundary. Where they do not share a boundary, they are usually in point contact across a junction between adjacent polygons.

3) In those intervals where the bar/channel lithofacies occurs in several drill holes, the pattern formed is a single curvilinear or branching band, and in the majority of these cases, the trend is continuous.

4) Where a trend established by the bar/channel lithofacies is interrupted, the intervening area is usually occupied by the levee/splay lithofacies. In those cases where the curvilinear bar/channel lithofacies trend ends abruptly, it can usually be continued through a series of levee/splay deposits.

5) Where isolated depocenters occur, they are usually composed of the levee/splay lithofacies and are usually separated from a curvilinear trend by a narrow, zone occupied by the coalswamp/overbank lithofacies.

Although the precise relationship between the accumulation of sediments within the study area and the passage of time has not been established, it is clear that each successive mapped interval is younger than the interval below it. If the generalizations listed above are valid and 
if they result from systematic changes in the geometry of the depositional system over time, then it is possible to link successive depositional patterns into a continuous series. If the depositional patterns of successive intervals are systematically compared, therefore, the sequential development of the depositional system in the study area emerges.

A comparison of the patterns taken in their stratigraphic context reveals several general trends. The most obvious of these is an overall increase, from the base of the section to the top, in the proportion of the area covered by bar/channel deposits. This increase occurred at the expense of the coalswamp/overbank lithofacies, as the proportion of the levee/splay lithofacies varies little over the section. Also evident is the general tendancy towards increased stability from the base of the section upwards. Less change occurred from interval to interval in the upper portion of the section than in the lower portion. Those changes which did occur were less drastic as the system developed.

In order to compare the depositional patterns among successive intervals, the curvilinear belts in each interval have been reduced to lines constructed along the centerline of each belt (Figure 34). This device reduces the complexity of the patterns, facilitating analysis. Although there is no progressive relationship between patterns which 

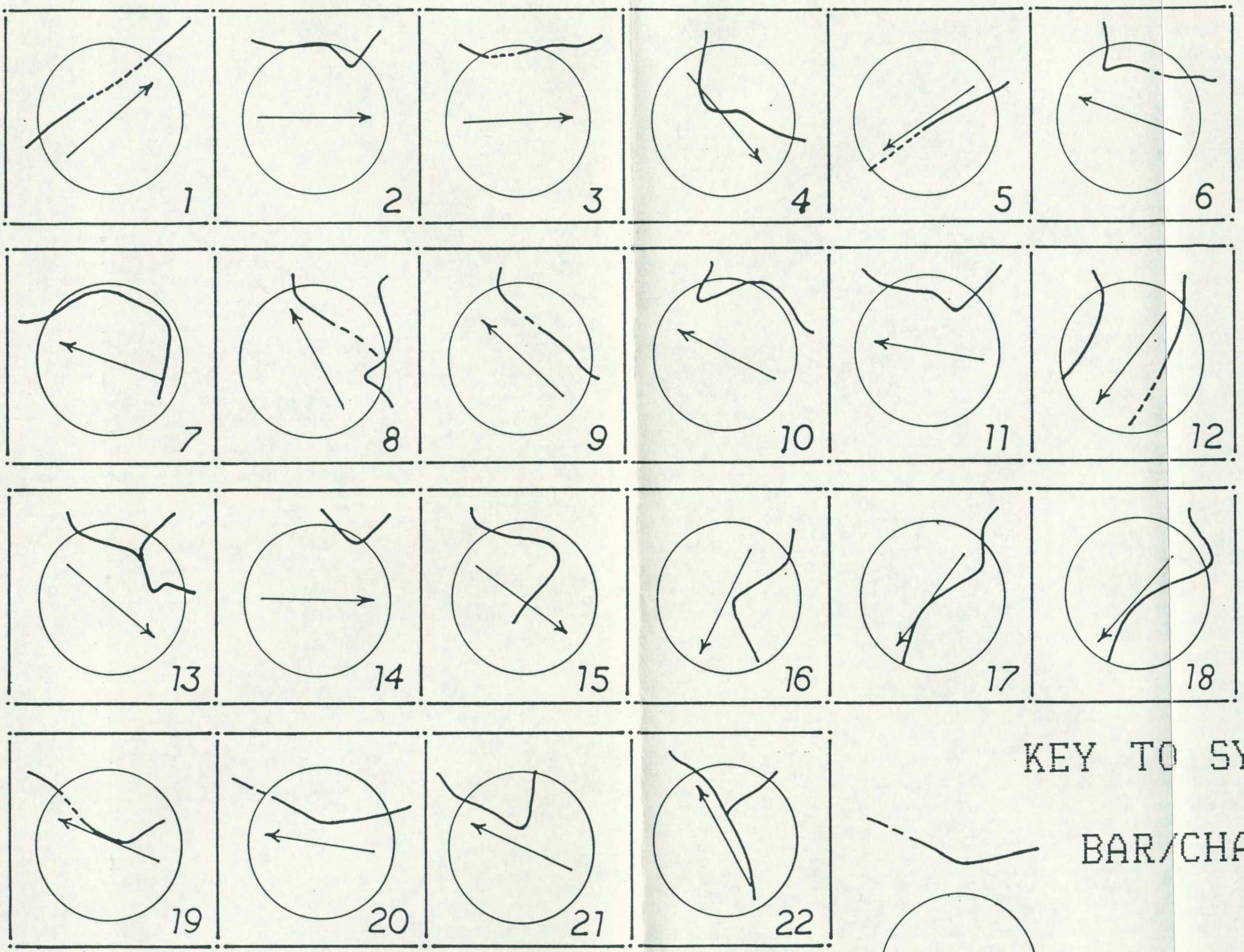

KEY TO SYMBOLS

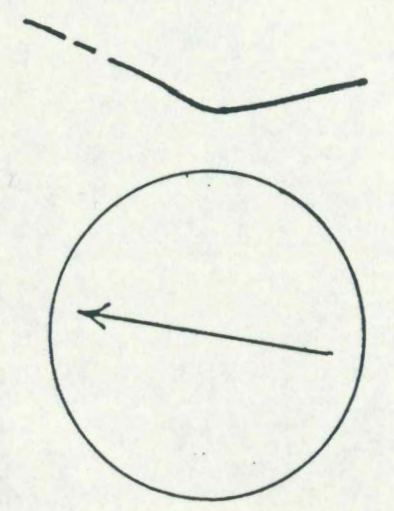

BAR/CHANNEL TREND

GENERALIZED AZIMUTH 
persists over the entire section, several periods of relative stability appear to be separated from one another by periods of reorganization. During intervals $1-3$, curvilinear belts are concentrated in the northern portion of the study area and oscillate about an east-west trend. Following a period of reorganization (intervals 3-6), this trend was re-established (intervals 6-7), the belts gradually migrating into a northwest-southeast trend (intervals 8-10) and back to their original position (intervals 11-15). A northeast trend was then established (intervals $15-17$ ). This trend gradually rotated into an east-west position slightly south of the initial trend.

In order to test these generalizations, the information contained in Figure 34 was further simplified. For each of the intervals, an estimate of the azimuth of its trend was made (Table 4). The resulting sequence was examined for systematic stratigraphic progressions. Since any of these trends may be described by either of two opposite azimuths, two separate analyses were made. The direction for each subsequent trend was chosen to minimize angular departure. For the following analysis, the first data set (Figure 35) was chosen, as its mean direction, 68\% confidence interval, and modal interval compare favorably with the paleocurrent directions obtained from measurements of primary sedimentary structures within the study area. Examination of Figure 35 a reveals the similarity 
TABLE IV

ESTIMATES OF BAR/CHANNEL AZIMUTHS,

CHINA CREEK AREA

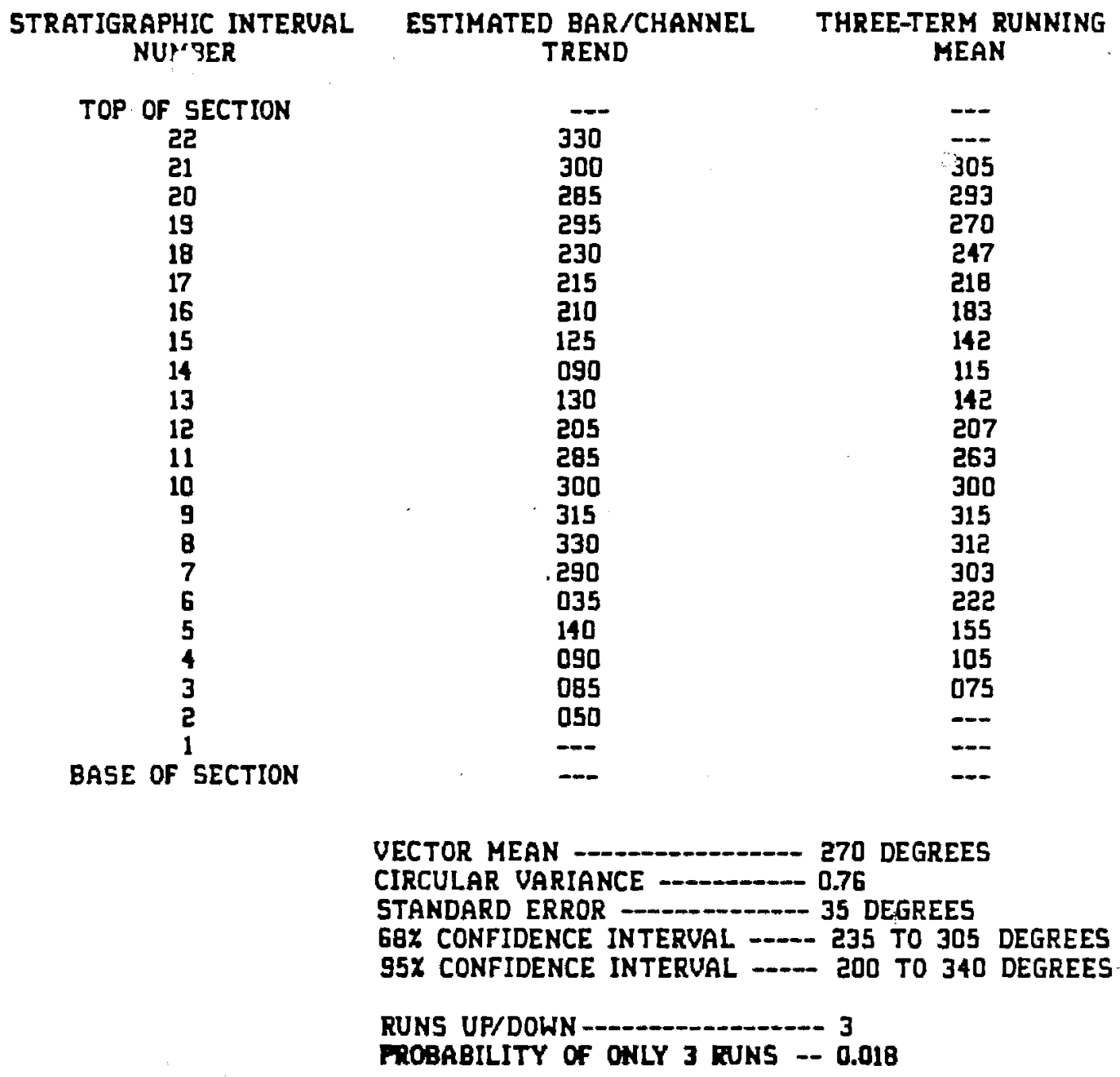




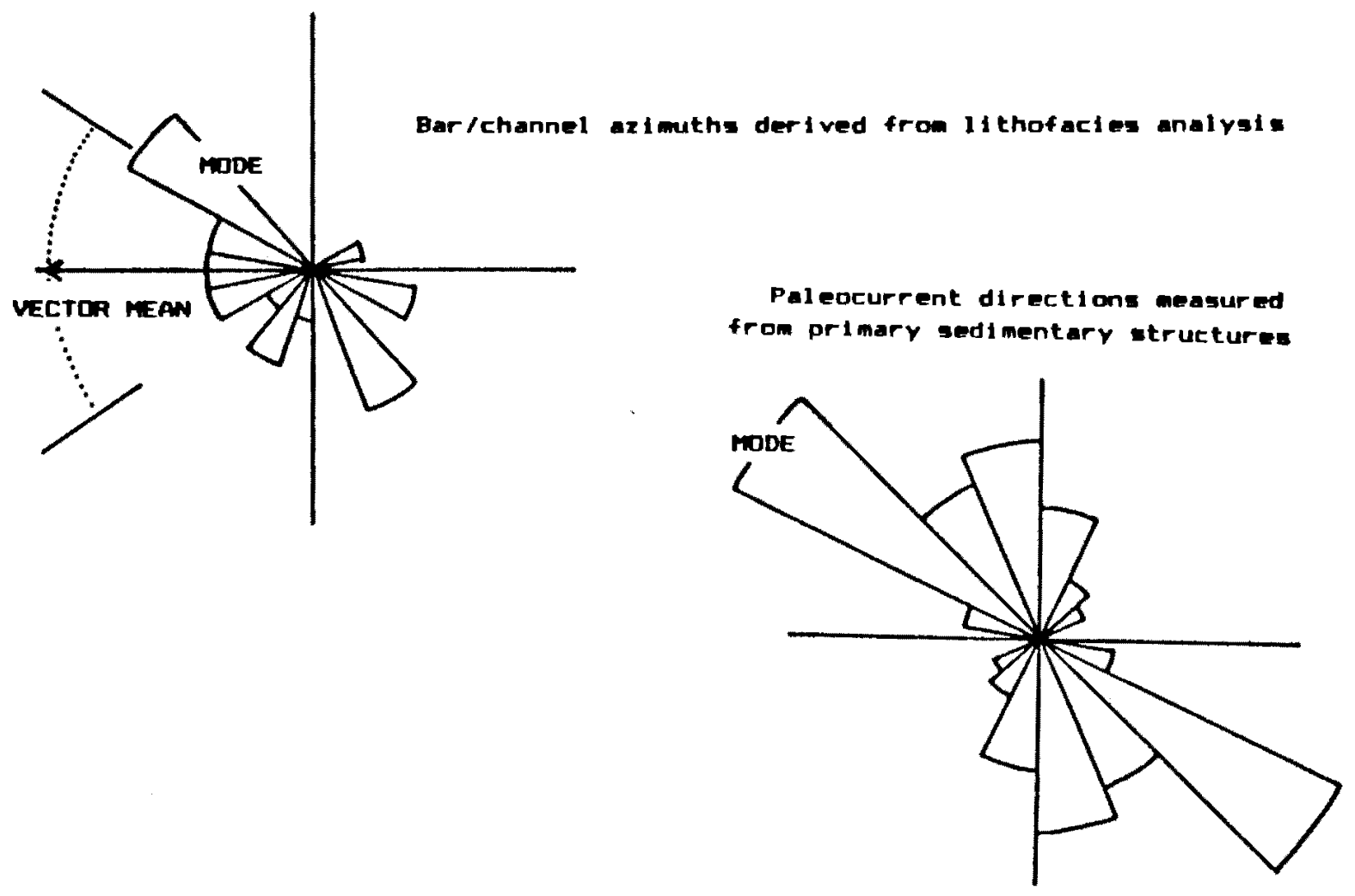

Figure 35. Comparison of bar/channel azimuths and paleocurrent directions, China Creek area.

$\stackrel{\circ}{\circ}$ 
among the trend estimates. A strong modal direction is indicated, $36 \%$ of the trends falling in 118 of the compass rose. Systematic stratigraphic progressions among the estimated trends were examined by plotting them against stratigraphic position (Figure 36 ). The sinusoidal nature of the sequence of smoothed values is obvious. Fourier analysis of the sequence results in the power spectrum shown in Figure 37. Significance testing indicates that the essential wavelength corresponds to 180 feet, approximately one third of the thickness of the stratigraphic section under investigation. Fitting the sequence with a three-harmonic Fourier series (Figure 38 ) results in a correlation coefficient of 0.91 and a goodness of fit of 0.82 , which is significant at the 99.58 confidence level. The data is consistent, therefore, with the hypothesis that the sequence of trends was systematic and varied sinusoidally, reversing four times from the base of the section to the top.

\section{COMPARISON OF LITHOFACIES DISTRIBUTIONS}

It is interesting to compare this model, based on the generalized frequency of occurrence and idealized sequences of lithofacies, to the series of actual facies maps of the area produced by direct analysis of geophysical logs of individual drill holes.

A qualitative estimate of the 


\section{STRATIGRAPHIC CHANNEL AZIMUTH VARIATIONS}

CHINA CREEK STUDY AREA, COOS BAY COAL FIELD

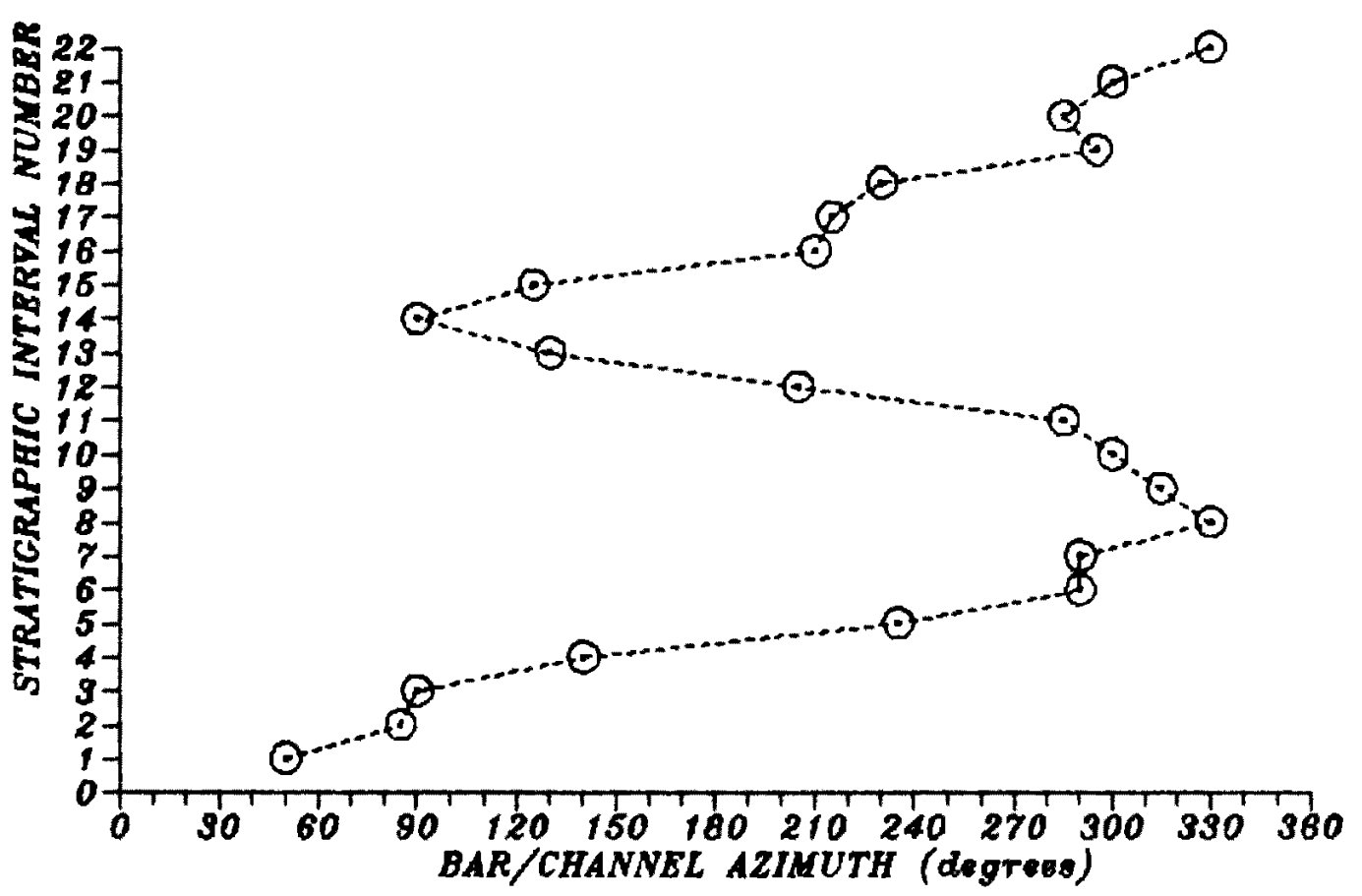

Figure 36. Stratigraphic channel azimuth variations, China Creek study area, Coos Bay coal field. 


\section{FOURIER ANALYSIS, BAR/CIIANNEL AZIMUTISS DISCRETE POWER SPECTRUM}

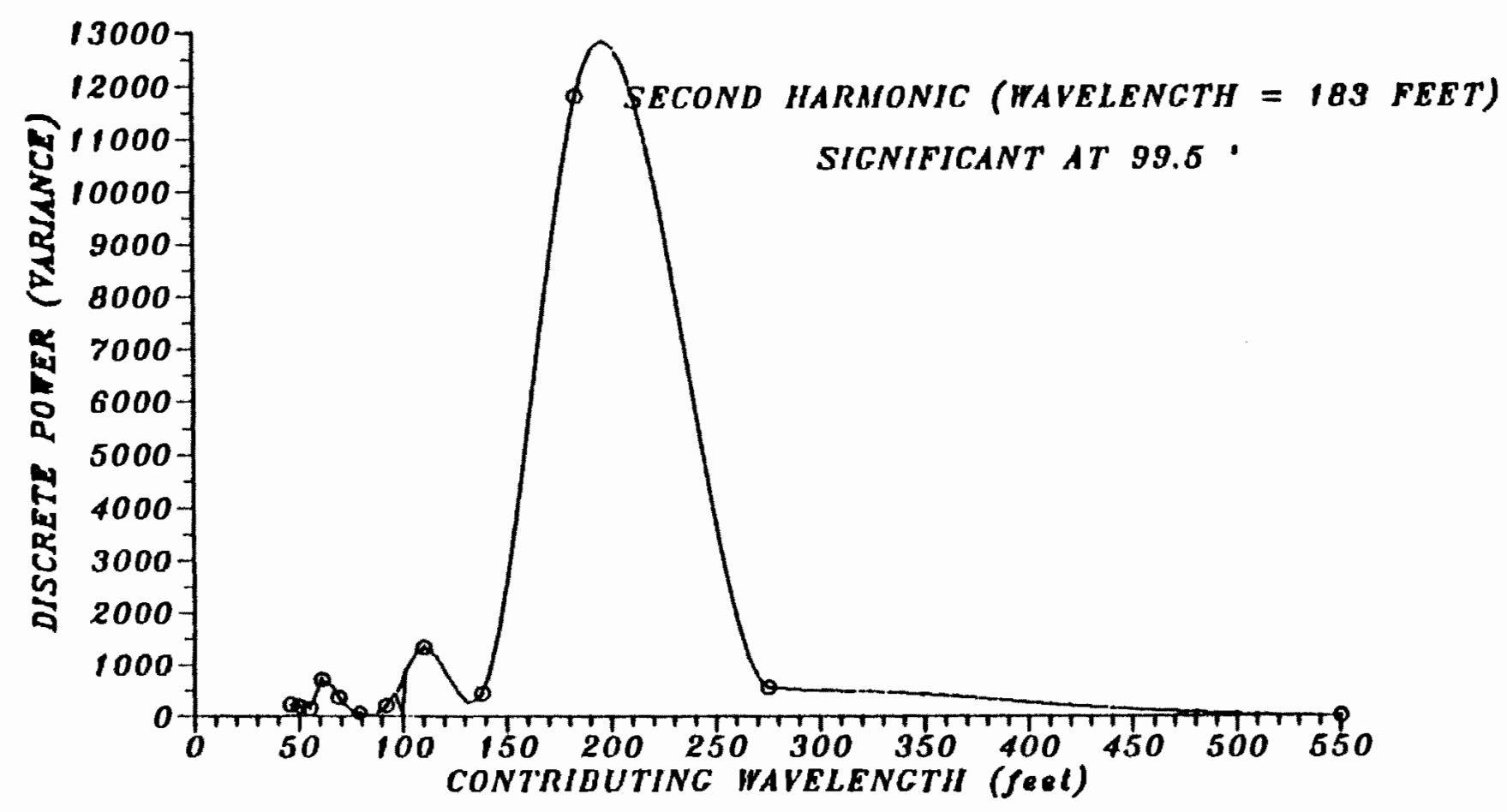

Figure 37. Fourier series power spectrum of bar/channel azimuths. 


\section{STRATIGRAPHIC CHANNEL AZIMUTH VARIATIONS}

CHINA CREEK STUDY AREA, COOS BAY COAL FIELD

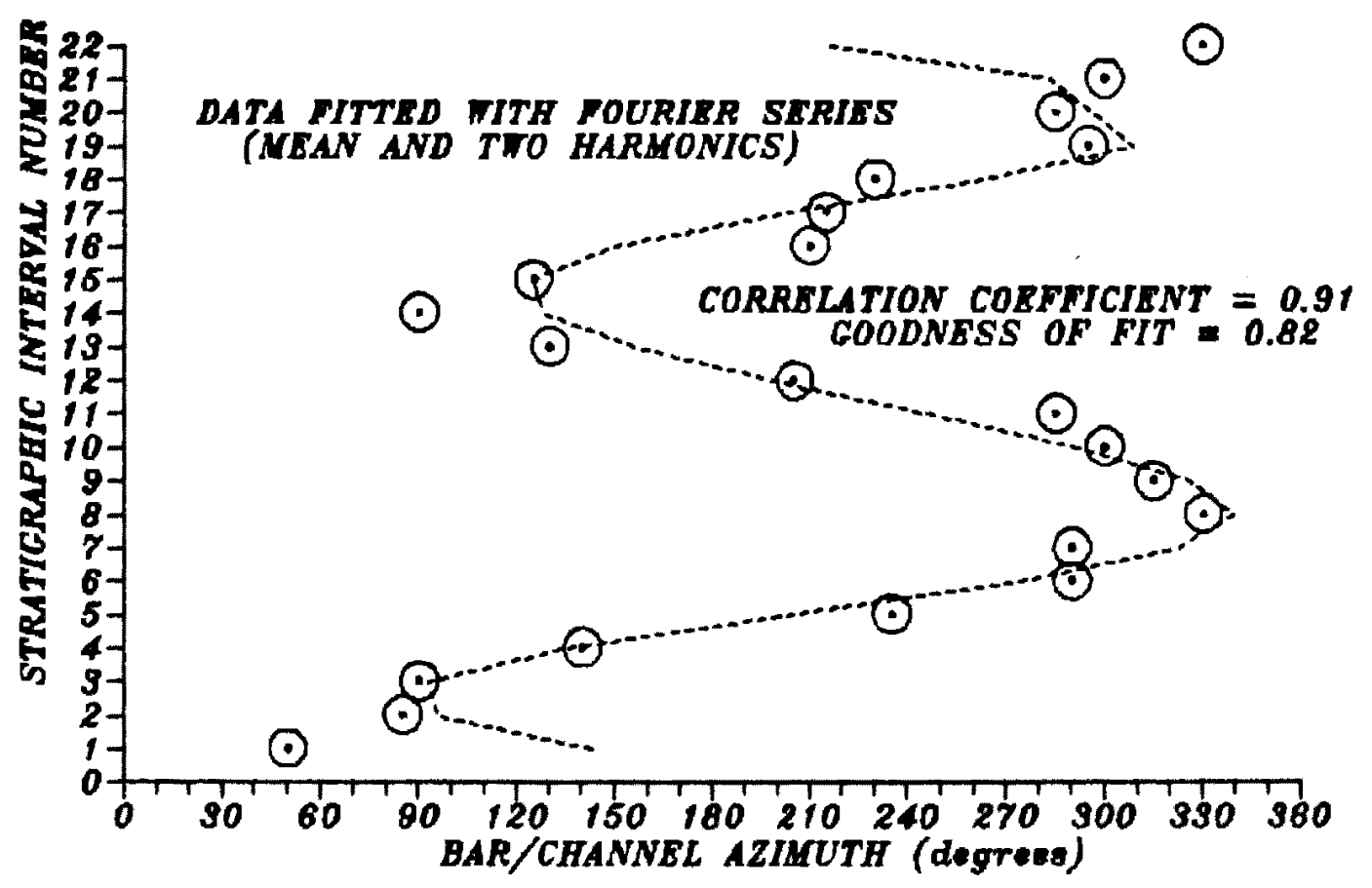

Figure 38. Three-harmonic Fourier fit to China creek channel azimuth data. 
similarity between the model and the actual facies distributions can be made by superimposing the two in several different orientations, as has been done in Figure 39. Examination of the results of the superimpositions leads to the conclusion that distortion of linear patterns and the generation of false contacts between units occurs, but little essential information is lost.

A comparison of the behavior of the bar/channel trends in the study area with the behavior of channel trends in modern meandering streams reveals several similarities. Figure 40 shows the channel pattern of a stretch of Watts Branch, a small meandering stream in Maryland (Leopold, 1973). Figure 41 summarizes the downstream distribution of thalweg azimuths over 27 consecutive intervals, each approximately 25 feet long. Figures 42 and 43 show the results of a similar study of a 35 kilometer $(22$ mile) reach of the lower Mississippi River near Jackson Point, Louisiana (Russell, 1967). The geometry of the two systems are similar, despite the order-of-magnitude differences in scale. The mean directions of the Watts Branch and lower Mississippi River data sets are excellent approximations of the transport directions of the streams, an indication that the logic used in selecting between the complementary azimuth sets in the study area is reasonable. The similarity between the downstream progression of channel azimuths along the modern meandering streams and the 

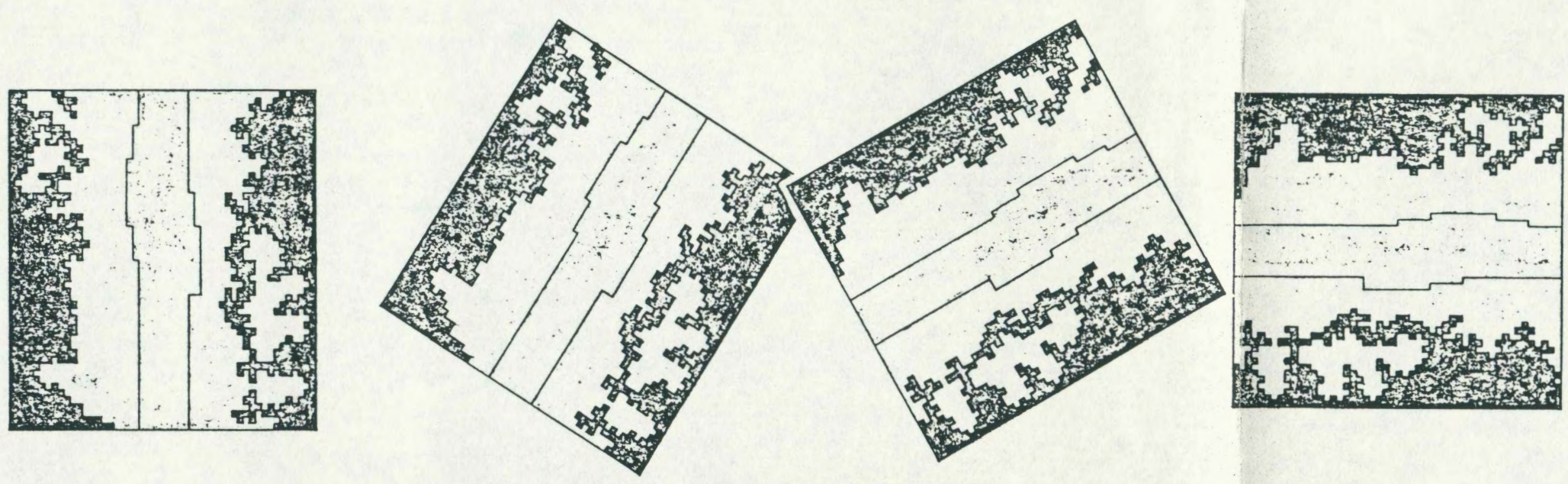

\section{LITHOFACIES MODEL}
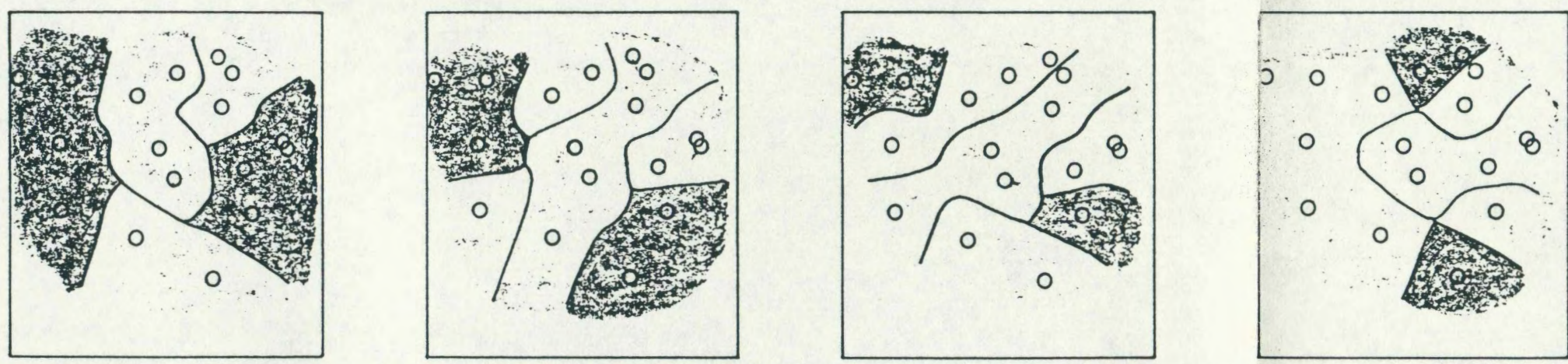

DERIVED LITHOFACIES MAP
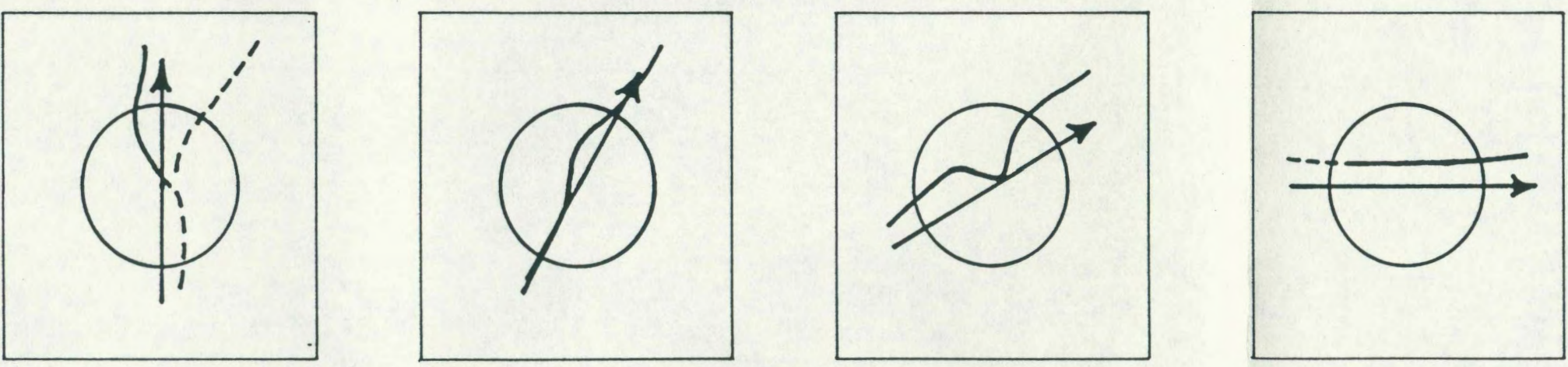

ESTIMATED AND TRUE BAR/CHANNEL TEENDS 


\section{DOWNSTREAM CHANNEL AZIMUTH VARIATIONS \\ WATTS BRANCH, MARYLAND}

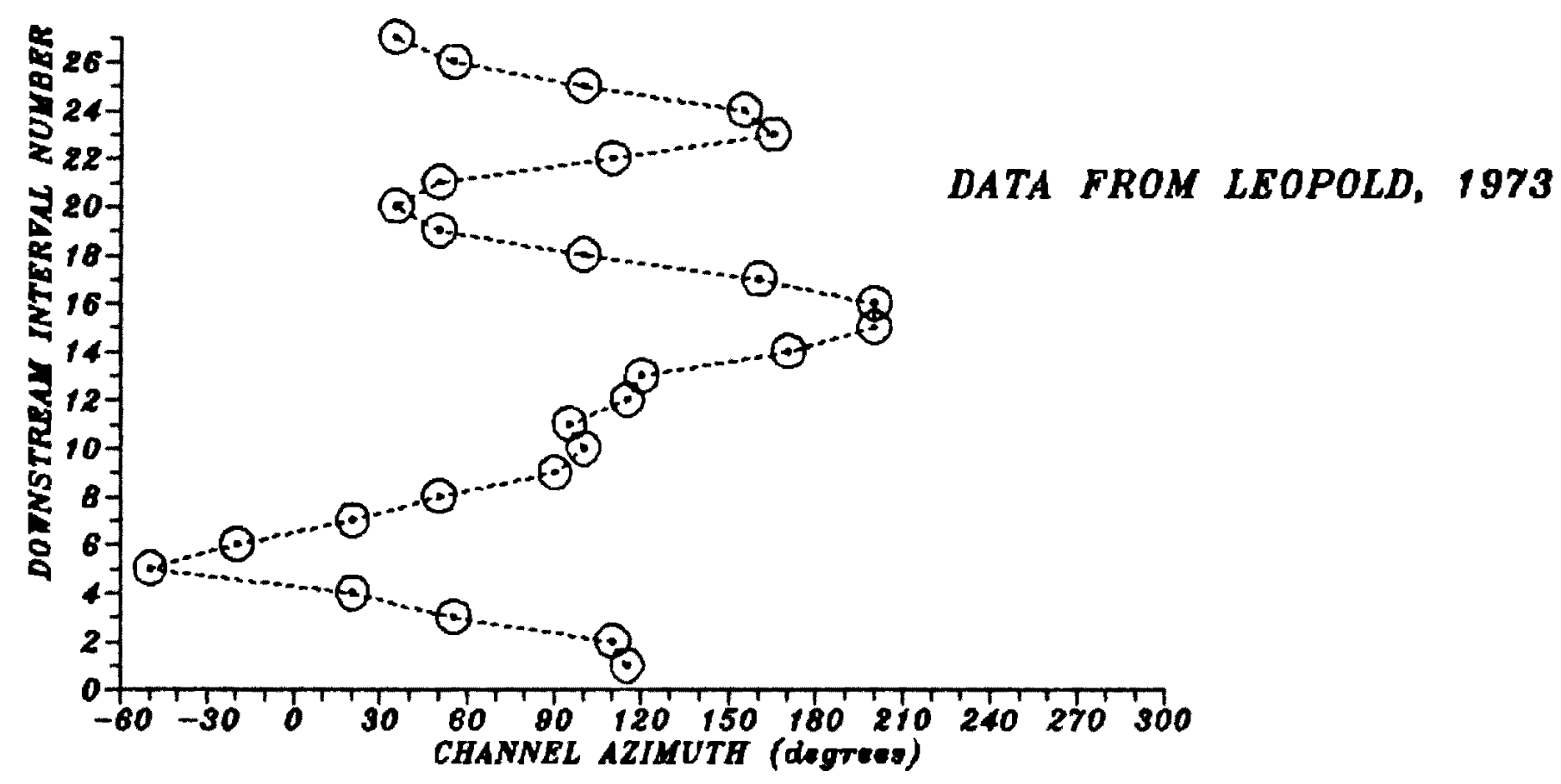

Eigure 40. Downstream channel azimuth variations, Watts Branch, Maryland. 


\section{CHANNEL AZIMUTHS}

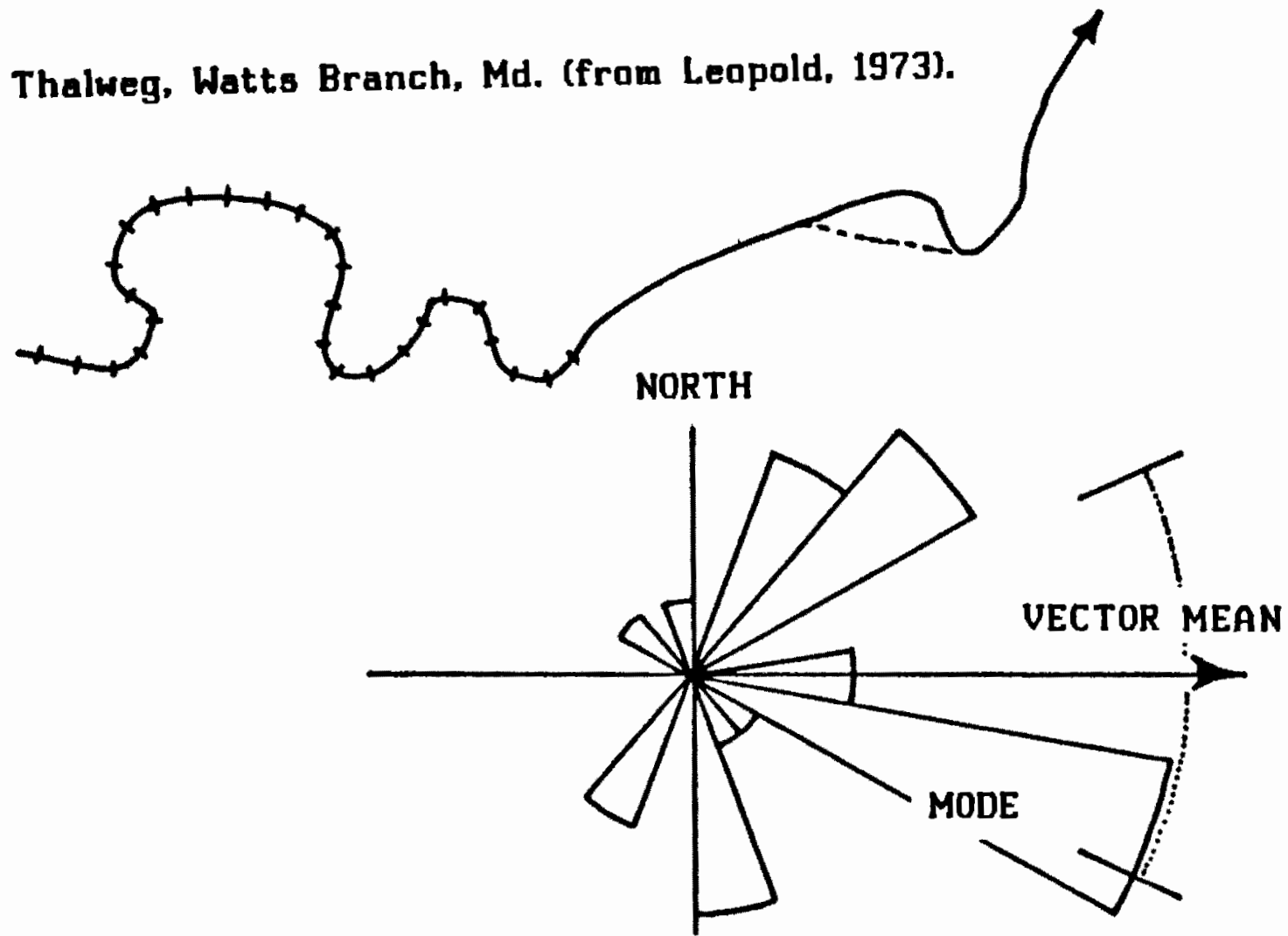
115
110
055
020
310
340
020
050
090
100
095
115
120
170
200
160
100
050
035
050
110
165
155
100
055
035

Rose diagram of thalweg azimuths, Watts Branch. Md. 
Roge diagram of thalweg azimutho, Lower Misgissippi River near Jackson Point. Louisiana.

CHANNEL AZIMUTIIS
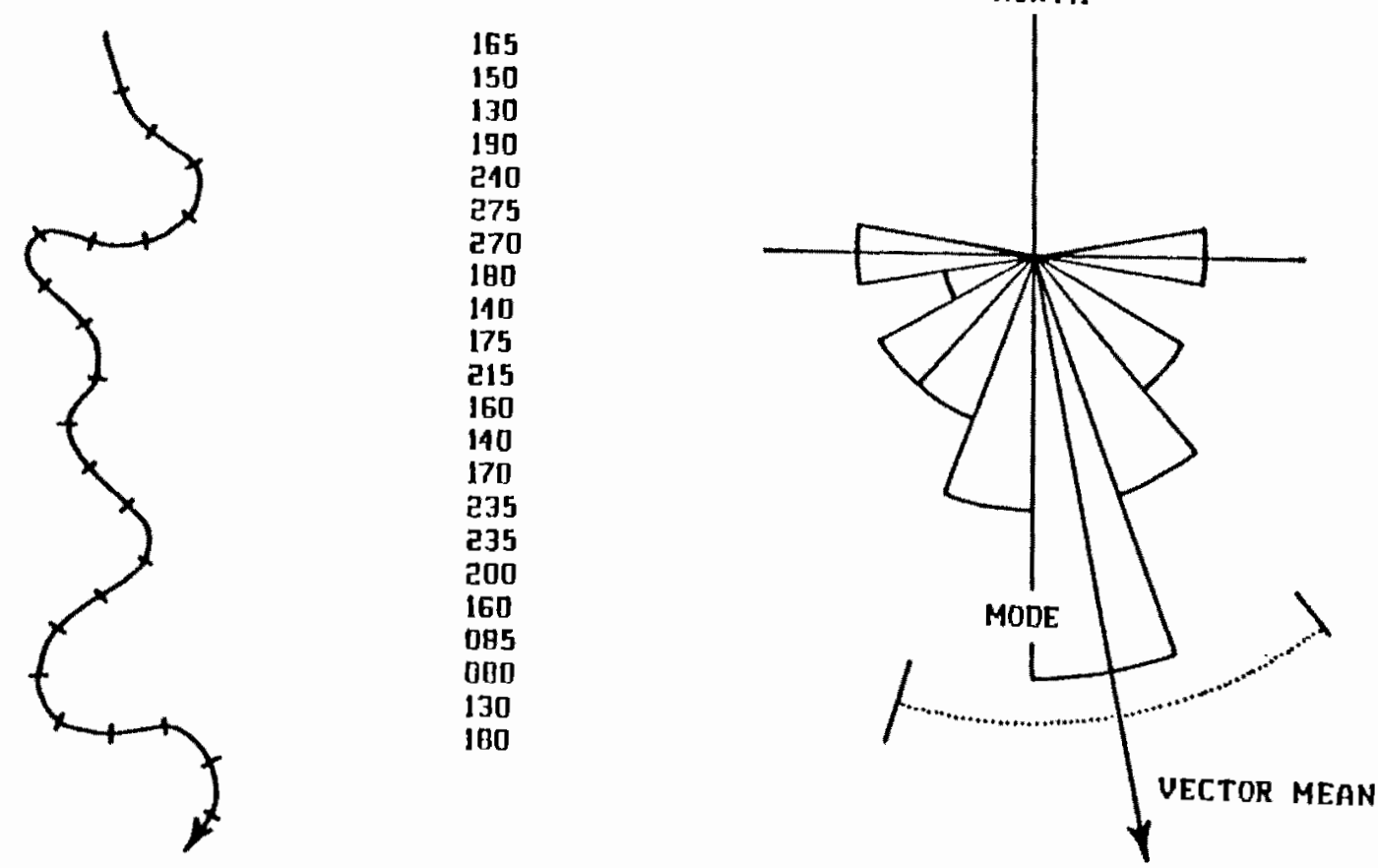

Thalweg. Lower Missigsippl Rluer near Jackson Point, Loulsiana (from Russell, 1967).

Fiqure 42. Thalweg azimuths, Lower Mississippi River near Jackson Point, Louisiana. 


\section{DOWNSTREAM CHANNEL AZIMUTH VARIATIONS LOWER MISSISSIPPI RIVER JACKSON POINT, LOUISIANA}

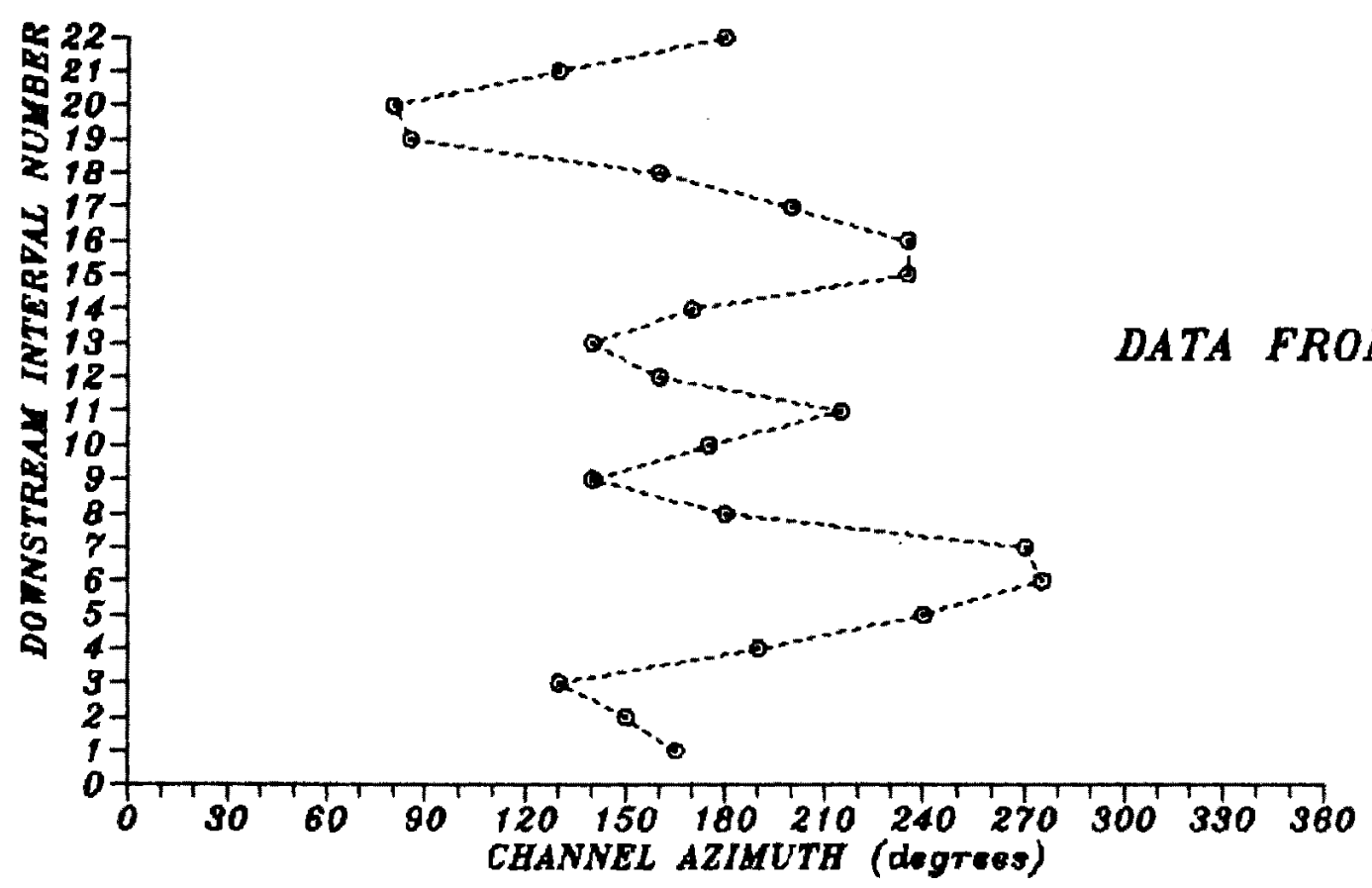

Figure 43. Downstream variations in channel azimuths, Lower Mississippi River. 
stratigraphic progression of bar/channel azimuths in the study area invites consideration of a mechanism by which the channel patterns of an aggrading, meandering stream may be preserved as a vertically stacked stratigraphic section.

The mechanism by which stratigraphic variations in the sequence of bar/channel azimuths in the study area may be linked to downstream variations in thalweg azimuths along the modern analogs involves the developmental behavior of aggrading, meandering streams. Although this aspect of fluvial behavior has not been studied over appreciable spans of geologic time, the character of the processes involved may be inferred from a short-term study of the Vermilion River, an aggrading, meandering stream near Eugene, Indiana (Jackson, 1980) and a study of flood plain development along the Little Missouri River near Watford City, North Dakota (Everitt, 1968). Figure 44 shows the location of the thalweg of a stretch of the Vermilion River, mapped on four occasions spanning thirty years. of particular interest is the tendancy for meanders to grow and migrate downstream. If the development and downstream migration of meander loops were to continue over a protracted period and if continuous deposition by vertical accretion were to occur during that same period, then the sequence of channel azimuths over a stationary point within the meander belt of the stream would vary in the same fashion as the downstream sequence of azimuths. These same tendancies are shown in the flood 


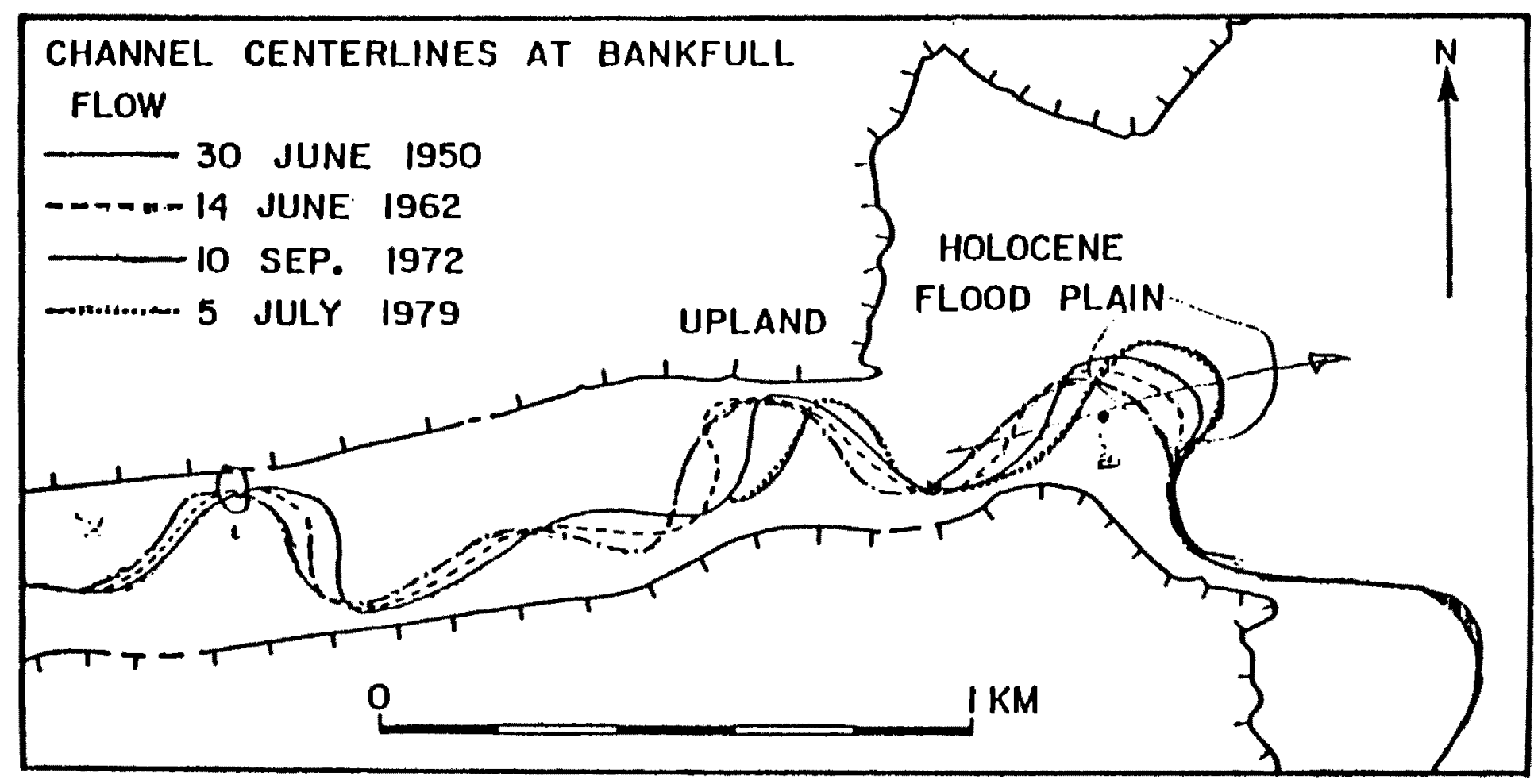

Figure 44. Sequential development of a channel pattern, Vermilion River, Indiana. 
plain age distribution map of a stretch of the Little Missouri River which Everitt (1968) produced by contouring the ages of cottonwood trees within the floodplain (Figure 45). 


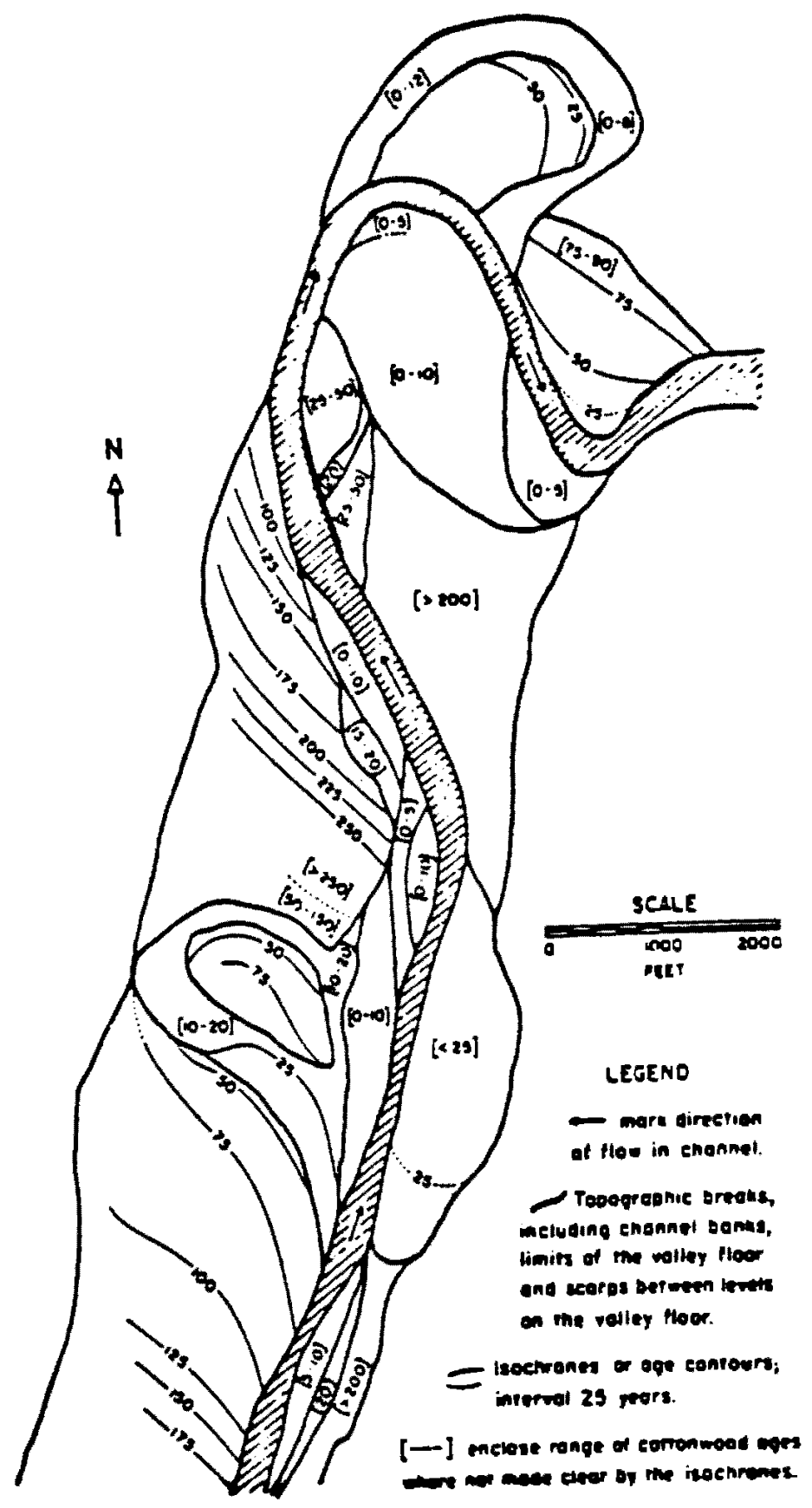

Figure 45. Sequential development of a flood plain, Little Missouri River. 
CHAPTER IV

\section{CONCLUSIONS}

The application of quantitative techniques to the stratigraphic analysis of data derived from geophysical log suites is both practical and effective. The benefits of this type of analysis include the high resolution of the results, the ability to derive fundamental lithological properties rapidly, directly from geophysical logs and simple granulometric analysis, the stratigraphic continuity and quantitative nature of the results, and the ease with which the results may be tested.

The resolution of the techniques used in this study, is an order of magnitude better than can be obtained from a combination of surface mapping, the projection of scattered measured sections and a qualitative inspection of the geophysical logs (Figure 46). Not only can very thin (less than 1 meter) strata be recognized, but subtle textural differences between strata can be detected. The results, moreover, are both measureable and reproducible, and do not include a significant personal operator bias.

The results of the initial log interpretation 
CROSS SECTION THROUGH THE CHINA CREEK AREA HUNG ON THE " $"$ " SEAM

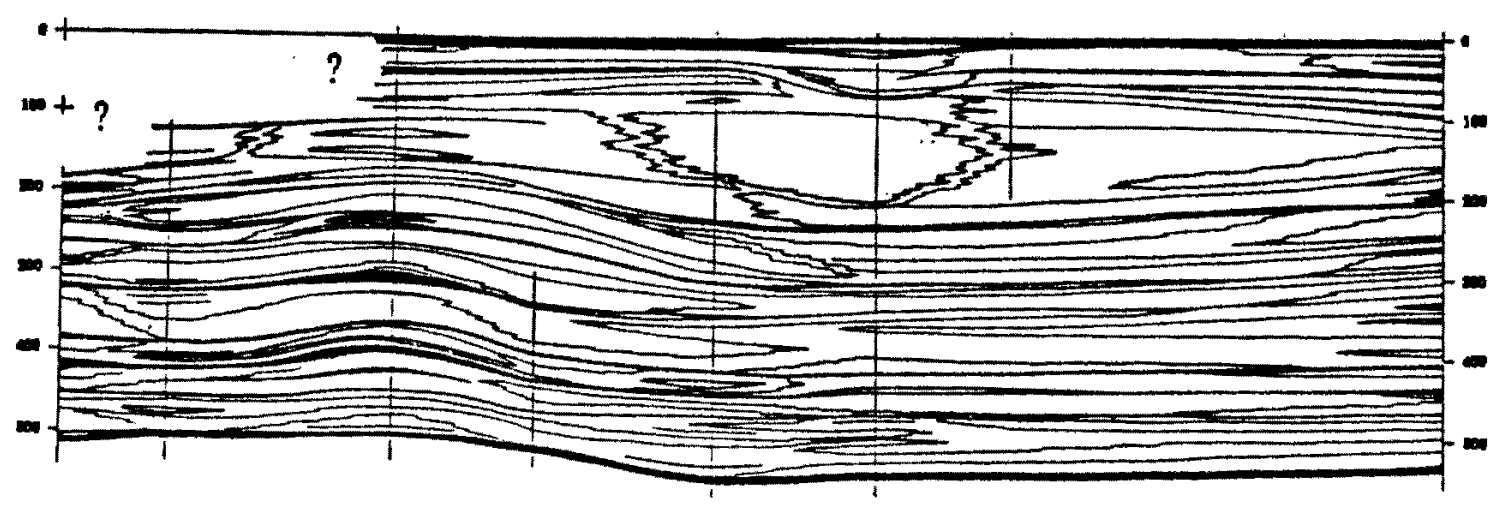

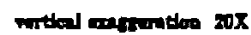

$\square \cos$

D FACTES A DLTAL OVERBANK DEPOSTTS

D FACTS I PROTOULL OVERBANK DEPOCTS

$\square$ PACTS C PONT DAR DEFOSTIS

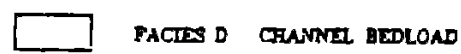

Figure 46. Example of the resolution of the quantitative stratigraphic analysis. 
technique is the "environmental energy index", which has been demonstrated to correlate with median grain size, a primary and extremely useful property of sedimentary rocks. Calibration of the technique is based on simple granulometric analyses of the cuttings produced during normal drilling operations. The technique, moreover, does not require exotic or expensive data processing equipment. All of the data processing for this study was carried out on a desktop microcomputer. The lithology interpretation program is capable of producing foot-by-foot environmental energy index logs and lithological interpretations of a 330 meter (1000 foot) geophysical $\log$ in less than ten minutes. Since modern logging vehicles are equipped with microcomputers of normal capacity, and produce digitized log response files, the logs could be interpreted on-site, as they are produced.

The log interpretation technique was developed and calibrated specifically for the rocks present in the study area and the general application to other rock suites in other areas has not been addressed. Since the grain size index is sensitive to a combination of factors, including modal composition, it is probably not directly applicable to rock types substantially different from those present in the study area. Since, however, the technique incorporates an internal calibration process, by which the grain size index values are scaled to match the results of a series of 
granulometric analyses of cuttings of the rocks being drilled, the technique is self-correcting to some extent. Provided, therefore, that the basic underlying assumptions of relatively stable quartz/feldspar and potassium feldspar/plagioclase ratios, uniform mode of occurrence of clay in the fabric of the rocks and low ionic concentrations in the pore water in the section being drilled are not violated, the technique should be applicable to a wide variety of unaltered sedimentary rocks.

The stratigraphic continuity and the quantitative nature of the initial lithologic interpretation allow the application of a variety of analytic techniques. Those whose suitability and efficacy have been demonstrated here include:

1) smoothing techniques, which can identify functional lithofacies,

2) Markov analyses of lithofacies transition probabilities, which can be used to identify the sequential patterns among depositional packages, 3) Walther's Law, which can be used in conjunction with calculated transition probabilities to infer the three-dimensional architecture of the deposits, and

4) sequential lithofacies map interpretation and spectral and vector analysis which can be used to infer the behavior of depositional systems over time. 
Many other analytical techniques could be applied to the data sets produced by these processes. The rapid advances currently being made in the analysis of time series and spatial distributions seem particularly applicable.

One of the most attractive features of the quantitative approach used in this study is the degree and ease with which its results can be tested. As has been demonstrated, testing is not limited to the final conclusions reached by the sequential application of many techniques. The results of each individual technique can be subjected to statistical test procedures before the next level of abstraction is attempted.

The resolution and flexibility of the stratigraphic information and the speed at which it can be produced by the techniques demonstrated in this study suggest a number of practical applications. Correlation of lithologic sequences within stratigraphically complex systems, calibration of remote subsurface sensing techniques (seismic, gravity, magnetic, etc), exploration for coal, petroleum and mineral resources, and the understanding of ground water and contaminant transport could benefit directly from the techniques. 


\section{REFERENCES CITED}

Allen, J.E. and Baldwin, E.M., 1944, Geology and coal resources of the coos Bay quadrangle: oregon Department of Geology and Mineral Industries Bulletin 27. $160 \mathrm{pp}$.

Anonymous, 1972, Log interpretation, Part I-Principles: Schlumberger Limited, New York, 113 pp.

Anonymous. 1982, Well logging and interpretation techniques: Dresser Atlas, Dresser Industries, Oklahoma City, 428 pp.

Armentrout, J.M., Hull, D.A., Beaulieu, J.D. and Rau, W.W.. 1983, Correlation of Cenozoic stratigraphic units of western Oregon and Washington: Oregon Department of Geology and Mineral Industries $O i l$ and Gas Investigation 7, 90 pp.

Armentrout, J.M. and suek, D.H., 1985, Hydrocarbon exploration in western Oregon and Washington: American Association of Petroleum Geologists Bulletin, vol. 69, no. 4, pp. 627-643.

Baldwin, E.M., 1976, Geology of Oregon: Kendall/Hunt, Dubuque, Iowa, 147 pp.

Baldwin, E.M. and Beaulieu, J.D., 1973, Geology and mineral resources of Coos County, Oregon: Oregon Department of Geology and Mineral Resources Bulletin 80, 82 pp.

Blatt, H., Middleton, G. and Murray, R,, 1980, Origin of sedimentary rocks: Prentice-Hall, Englewood Cliffs, New Jersey. $782 \mathrm{pp}$.

Carr, D.D., Horowitz, A., Hrabar, S.V., Ridge, R.F., Rooney, R., Straw, W.T., Webb. W. and Potter, P.E., 1965, stratigraphic sections, bedding sequences and random processes: Science, vol. 154, pp. 1162-1164.

Chan, M.A., 1982, Comparison of sedimentology and diagenesis of Eocene rocks, southwest Oregon: Madison, Hisconsin, University of Visconsin Ph.D. dissertation, 311 pP., unpublished.

Chan, M.A. and Dott, R.H., 1986, Depositional facies and progradational sequences in Bocene wave-dominated deltaic complexes, southwestern Oregon: American 
Association of Petroleum Geologists Bulletin, vol.

70, no. 4, pp. 415-429.

Crowell, J.C., 1974, Origin of late Cenozoic basins in southern California: Society of Economic

Paleontologists and Mineralogists Special Publication 22 , pp. 190-204.

Davis, J.C., 1986, Statistics and data analysis in geology: John Wiley \& Sons, New York, $646 \mathrm{pp}$.

Diller, J.S., 1899, The Coos Bay coal field, Oregon: Nineteenth Annual Report of the United States Geological Survey (1897-1898), Part III (Economic Geology), pp. 309-376.

Diller, J.S., 1901, Description of the Coos Bay Quadrangle: United States Geological Survey Geological Atlas of the United States, Folio 73.

Diller, J.S. and Pishel, M.A., 1909, Preliminary report on the coos Bay coal field, Oregon: United States Geological Survey Bulletin 431, Part II, pp. 190-228.

Dott, R.H., 1966, Eocene deltaic sedimentation at Coos Bay, Oregon: Journal of Geology, vol. 74, no.4, pp. 373-420.

Doveton, J.H., 1986, Log analysis of subsurface geology: concepts and computer methods: John Wiley \& Sons, New York, 273 pp.

Duncan, D.C., 1953, Geology and coal deposits in part of the Coos Bay coal field, Oregon: United States Geological Survey Bulletin 982-B, $73 \mathrm{pp}$.

Everitt, B.L., 1968, Use of the Cottonwood in an investigation of the recent history of a flood plain: American Journal of Science, vol. 266, pp. 417-439.

Gingerich, P.D., 1969, Markov analysis of cyclic alluvial sediments: Journal of Sedimentary Petrology, vol. 39, pp. 330-332.

Heller, P.L. and Ryberg, P.T., 1983, Sedimentary record of subduction to forearc trandition in the rotated

Eocene basin of western Oregon: Geology, vol. 11, pp. $380-383$.

Jackson, R.G., 1980, Sedimentology of the Vermilion River (IIlinois and Indiana) and its significance for lithofacies models of meandering alluvial streams: 
Society of Economic Paleontologists and Mineralogists, Great Lakes Section Field Trip

Guidebook, Tenth Anual Meeting, September, 1980, pp. 17-44.

Leopold, L.B., 1973, River channel change with time: an example: Geological Society of America Bulletin vol. 84 , no. 6, pp. 1845-1860.

Leopold, L.B., Wolman, M.G. and Miller, J.P., 1964, Fluvial processes in geomorphology: W.H. Freeman, San Francisco, 522 pp.

Mann, C.J., 1981, stratigraphic analysis: decades of revolution (1970-1979) and refinement (1980-1989); in Merriam, D.F. (ed.), 1981, Computer applications in the earth sciences: Plenum Press, New York, pp. 211-242.

McRee, B., 1972, Cascadia: the geologic evolution of the Pacific Northwest: McGraw-Hill, New York, 394 pp.

Robertson, R.D., 1982, Subsurface stratigraphic correlations of the Eocene Coaledo Formation, Coos Bay basin, Oregon: Oregon Geology, vol. 44, no. 7, pp. 75-78.

Rooth, G.H., 1974, Biostratigraphy and paleoecology of the Coaledo and Bastendorf Formations, southwestern Oregon: Corvallis, Oregon; Oregon State University Ph.D. dissertation, 270 pp., unpublished.

Russell, R.J., 1967, River plains and sea coasts: University of California Press, Berkeley, $173 \mathrm{pp}$.

Ryberg, P.T., 1978, Lithofacies and depositional environments of the Coaledo Formation, Coos County, Oregon: Eugene, Oregon; University of Oregon M.Sc. thesis, 159 pp., unpublished.

Snavely, P.D. and Wagner, H.C., 1963, Tertiary geologic history of western Oregon and Washington: Washington State Division of Mines and Geology Report of Investigations $22,25 \mathrm{pp}$.

Vaninetti, G.E., 1978, Coal exploration concepts and practices in the western United States: Proceedings of the Second International Coal Exploration symposium, pp. 132-194.

Vaninetti, G.E., 1981, Geophysical well-logging and related subsurface data useful in coal exploration and development programs: American Association of 
Petroleum Geologists Short Course, steamboat Springs, Colorado, 1981, 83 pp.

Wyllie, M.R.J., 1963, The fundamentals of well log interpretation: Academic Press, New York, $238 \mathrm{pp}$. 